Portland State University

PDXScholar

Spring 5-24-2016

\title{
The Development and Validation of the Social Recovery Measure
}

\author{
Casadi "Khaki" Marino
}

Portland State University

Follow this and additional works at: https://pdxscholar.library.pdx.edu/open_access_etds

Part of the Social Work Commons

Let us know how access to this document benefits you.

\section{Recommended Citation}

Marino, Casadi "Khaki", "The Development and Validation of the Social Recovery Measure" (2016).

Dissertations and Theses. Paper 2925.

https://doi.org/10.15760/etd.2921

This Dissertation is brought to you for free and open access. It has been accepted for inclusion in Dissertations and Theses by an authorized administrator of PDXScholar. Please contact us if we can make this document more accessible: pdxscholar@pdx.edu. 
The Development and Validation of the Social Recovery Measure

by

Casadi Michelle Marino

A dissertation submitted in partial fulfillment of the requirements for the degree of

Doctor of Philosophy

in

Social Work and Social Research

\author{
Dissertation Committee: \\ Maria Talbott, Chair \\ Eileen Brennan \\ Mary Oschwald \\ Greg Townley \\ Melissa Thompson
}

Portland State University

2016 
(C) 2016 Casadi Michelle Marino 


\begin{abstract}
Mental health recovery is a complex phenomenon involving clinical, functional, physical, and social dimensions. The social dimension is understood to involve meaningful relationships and integration with supportive individuals and a wider community. While the recovery model developed from a movement led by consumers and survivors of the mental health system to promote hope, self-determination, and social inclusion, the clinical aspects of recovery have dominated mental health research and practice. The under-investigated area of social recovery calls for psychometrically sound measurement instruments. The purpose of the current study was to develop and validate the Social Recovery Measure (SRM). The study was grounded in disability and mad theories which locate disability at the intersection of the person and the environment. The SRM is a 19-item self-administered instrument scored on a 5-point Likert scale that consists of two domains: Self and Community.

Items for the SRM were developed through focus groups and interviews with 41 individuals in recovery from mental health challenges and the preliminary measure was administered to a purposive, nonprobability sample of 228 individuals in recovery. A confirmatory factor analysis (CFA) was conducted and a re-specified model resulted in good model fit. The SRM exhibited excellent internal consistency with a Cronbach's coefficient alpha of .951 and demonstrated excellent test-retest reliability, content validity, and construct validity.

Social recovery is highly relevant for social work given the discipline's commitment to disenfranchised populations and investment in creating enabling environments. The
\end{abstract}


SRM has utility for use in evidence based practice and evaluation. The SRM can be used to further research in social recovery, test underlying theory bases, and explore the differential effects of the multiple dimensions of recovery. There is a need to better understand social recovery which this measure can facilitate. 


\section{Acknowledgments}

I would like to recognize the members of my dissertation committee: Dr. Maria Talbott, Chair, Dr. Eileen Brennan, Dr. Mary Oschwald, Dr. Greg Townley, and Dr. Melissa Thompson.

I would like to acknowledge the members of my expert panel: Dr. Larry Davidson, Dr. Nev Jones, Stephania Hayes, and Candice Morgan.

I extend my appreciation to the mad grrls in the academy who supported me over the years.

I am grateful to Christina Aguilera for the song "Fighter" which I listened to repeatedly for motivation during the last stages of my $\mathrm{PhD}$ program.

Y gracias a las señoras de La Casita para los tacos vegetarian. Adios. 


\section{Table of Contents}

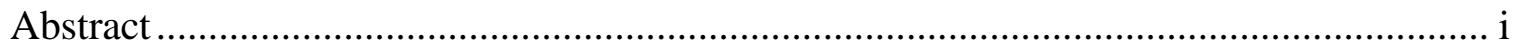

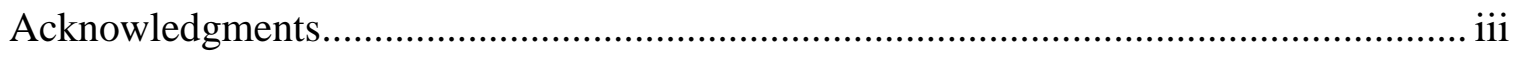

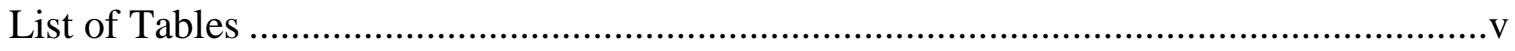

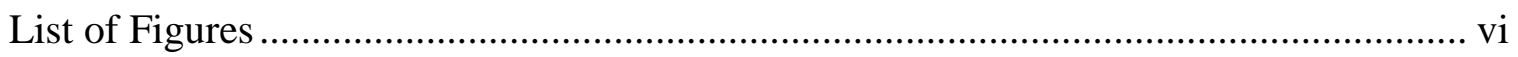

Chapter 1

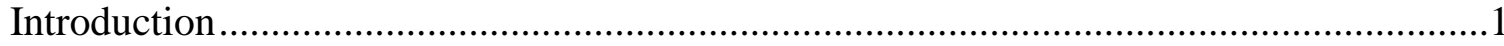

Chapter 2

Review of a Theory Base for and the Literature on Recovery ....................................13

Chapter 3

Methods .................................................................... 115

Chapter 4

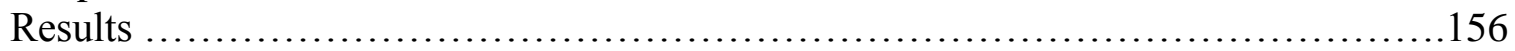

Chapter 5

Discussion .............................................................. 177

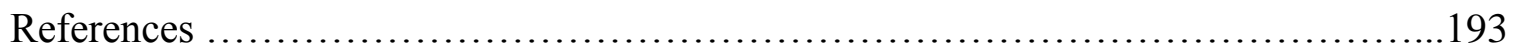

Appendices

Appendix A. IRB Approval ..................................................................231

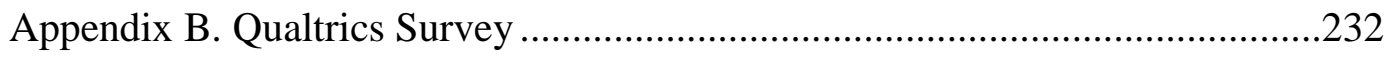

Appendix C. Skew and Kurtosis of the Items on the Draft SRM .....................246

Appendix D. Total Variance Explained and Pattern Matrix.................. 252

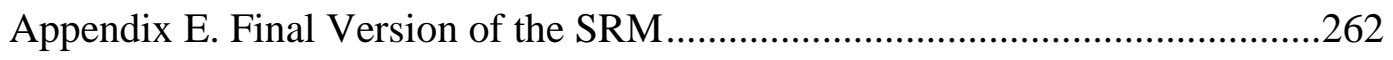




\section{List of Tables}

Table 1. Summary of Instrument Quality Ratings ......................................................110

Table 2. Demographic Characteristics: Residency, Age, Gender, Race/Ethnicity, Sexual

Orientation, Relationship Status, Living Situation .................................................157

Table 3. Demographic Characteristics: Education, Employment, Services Type,

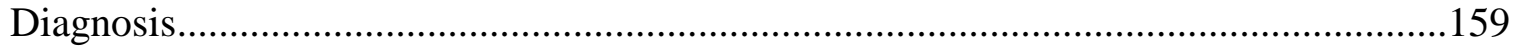

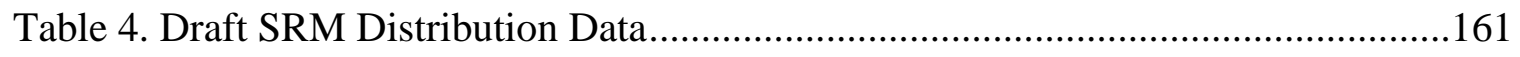

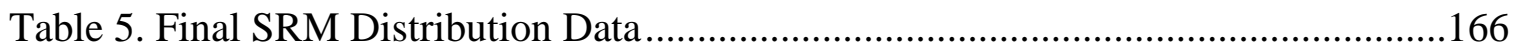

Table 6. Factor Loadings for Final SRM Items ....................................................167

Table 7. Correlation, One-way ANOVAs, and T-Test of Non-significant Findings.......175 


\section{List of Figures}

Figure 1: Scree Plot of Eigenvalues of Factors for Exploratory Factor Analysis ............164

Figure 2: 19-item Two Factor Model of the Social Recovery Measure (SRM) ..............168 


\section{Chapter 1}

\section{INTRODUCTION}

Mental health and illness have proven difficult to define. Concepts and values differ across social groups, cultures, and time periods. There is insufficient understanding of the etiology of mental illness. The difficulty with definitions has challenged the development of common approaches and effective interventions (Satcher, 2000). Mental health policy is fundamentally shaped by the prevailing definition of mental illness. Changing policies reflect changing definitions (Goldman \& Grob, 2006).

According to the Surgeon General, serious mental illnesses are understood as conditions that interfere with social functioning (U.S. Department of Health and Human Services, 1999). The Center for Psychiatric Rehabilitation defines "psychiatric disability" as mental illness which significantly interferes with performance of major life activities such as communicating with others and working (Center for Psychiatric Rehabilitation, 2012). Four percent or 9.6 million adults aged 18 or older in the U.S. have serious mental illnesses. Mental illness accounts for the largest proportion of disability in the United States as well as in other developing countries. The economic cost of mental illness in the United States is approximately 300 billion dollars a year (Centers for Disease Control and Prevention, 2011; National Institute of Mental Health, 2014). Individuals with mental health issues face significant financial issues and employment barriers. According to the General Accounting Office, major mental illness is found disproportionally among the poor and homeless. Approximately one-third of homeless adults in the United States are believed to have major mental illness (GAO, 2000). An estimated one-half of individuals 
with serious mental illness live at or near the poverty level (Cook, 2006). Nationwide, $10 \%-20 \%$ of individuals with mental illness are employed versus $70 \%$ of individuals without disabilities (Bureau of Labor Statistics, 2011; National Association of Mental Health Program Directors, 2007). Individuals with major mental illness are overrepresented in jails and prisons. Prevalence rates of serious mental illness are thought to be three to six times greater in the prison population than in the community (U.S. Department of Justice, 2007). Compared to the general U.S. population, public mental health clients die 25 years younger (Colton \& Manderscheid, 2006; Manderscheid, et al., 2010).

\section{Stigma}

Mental illness has been described as "the ultimate stigma" and a "mark of shame" (Green, 2009). Stigma is founded on a discredited individual difference and is characterized by lack of knowledge and fear (Corrigan, Watson, Byrne, \& Davis, 2005). Stigmatizing beliefs lead individuals with relative social power to restrict opportunities to individuals with a perceived difference (Corrigan, Bink, Fokuo, \& Schmidt, 2015). Given the pain caused by exclusion and rejection, stigma has been characterized as a form of social death (Corbiere, Samson, Villotti, \& Pelletier, 2012; Ralph, 2002). The self-stigma that results from internalization of negative public attitudes and stereotypes damages selfconcept and can lead to social withdrawal and hopelessness (Rusch et al., 2014). The World Psychiatric Association maintains that stigma and social exclusion are greater barriers to quality of life for mental health consumers than illnesses themselves. The National Association of Social Workers similarly regards stigma and role disability as 
key barriers to recovery. Stigma involves a vicious cycle of discrimination and social exclusion and consequences such as unemployment, lack of housing, and weak social support promote disability (NASW, 2015; WPA, 2016). It is important to maintain a focus on stigma as a social phenomenon. It is not an inherent attribute of an individual but a social construction imposed on an individual. Addressing stigma is not only a mental health matter but a social justice issue (Jones \& Corrigan, 2014). As stigma and discrimination function to prevent people from exercising their full rights as citizens and meeting their human needs for connection, the role of a service system should be to challenge stigma and work towards creating a society that promotes inclusion and wellbeing as well as treating illness and mental distress (Slade, 2010). A human rights-based interpretation of mental health recovery would recognize that disability results from interactions between people and attitudinal and environmental barriers. The human-rights stance would prioritize self-determination and social inclusion, in all aspects of support and services (Forrest, 2014). Social inclusion is not a new concept in the mental health field, but it has been increasingly emphasized as a key outcome in consumer-influenced recovery perspectives for services and policies. Social inclusion has been defined as the opportunity to participate in society as one wishes (Baumgartner \& Burns, 2014).

\section{History of the Social Problem}

In different cultures and historical periods, mental illness has been associated with supernatural powers, spiritual gifts or curses, imbalance of the physical, mental, and spiritual states, immorality, irrationality, and/or dangerousness (Beresford \& Campbell, 2005; Singh, 1999). Some groups have practiced banishing of individuals whose behavior 
was seen as threatening the order and functioning of the society. Mental illness has been conceptualized as deviations from norms for which the individual as well as his or her family or group would suffer negative social consequences (Cockerham, 1989; Eaton, 2001; Slate \& Johnson, 2008).

Before 1800, insanity in the U.S. was regarded as largely an individual matter, not as a significant medical issue or pressing social problem. Individuals were to be provided for by family, through poor laws, and by private charity, although some individuals became homeless and were forced to beg for a living. As the population grew denser in the early 1800s, institutions were created in response to community need. The majority of individuals regarded as insane were in local poor houses or jails. Cure was not sought (Durham, 1989; Grob, 1985). With increased industrialization and urbanization, the unmet needs of the mentally ill came to the public's attention. The insane came to be recognized as a distinct social category and there was a call for a governmental response. At the beginning of the nineteenth century, the insane were moved from jails and almshouses and established in asylums (Payne, 2009). During this time, the insane were regarded from moralistic or religious points of view and some saw them as sub-human (Deutsch, 1949). In addition to institutional confinement, treatments included: bleeding, purging, gyration, blistering, nausea, and seclusion (Skultans, 1975).

The Moral Model of treatment developed in the mid nineteenth century. The term "moral treatment" was borrowed from the French term "traitement moral" used by the French reformer Pinel. The French word "moral" is closer in meaning to the English term "morale" which concerns self-esteem and emotional well-being. Moral treatment was 
based on providing a sympathetic and supportive environment that would assist healing and adopted a psychological frame on insanity. The approach concentrated on the emotional causes of illness and maintained that insanity was curable. Basic principles included personal respect for people with severe mental health problems and an emphasis on the healing power of everyday relationships (Borthwick et al., 2001). An important component of the treatment was the practice of engaging in dialogue with patients and asking them about their lives, experiences, interests, and goals. The approach maintained that discussing and understanding behavior and emotions could lead to relief (Hamm, Hasson-Ohayon, Kukla, \& Lysaker, 2013; Morrissey \& Goldman, 1986).

Beginning in 1845, the pace of immigration into the U.S. began to increase.

Immigrants in poor health were directed to existing institutions including asylums. As no new funding was available, asylums became overcrowded and therapeutic practices were undermined. Moral treatment was abandoned and programs focused on custodial care (Miller \& Blanc, 1967). The medical model developed during this time period and mental health issues became regarded as illnesses (Cockherham, 1989; Eaton, 2001). In the early 1900s, individuals such as Clifford Beers who had been confined in asylums began writing and publically speaking about their negative experiences. The psychiatrist Adolf Meyer and psychologist William James used such accounts to establish the Mental Hygiene Movement. The movement focused on prevention and community-based care (Rothman, 1980). Psychiatric social work became recognized as a specialty during this time period and focused on securing resources for community living (Grob, 1983; Stuart, 1997). 
Psychoanalysis developed in the early 1900s as well. Freud maintained that people with serious mental illnesses could not engage in the treatment as they could not form a proper attachment to the therapist. His views were consistent with those of psychiatrists Bleuler who coined the word schizophrenia and Kraepelin who taught that schizophrenia and related conditions were chronic disorders with poor prognoses (Karon \& Widener, 1999). However, Jung and other practitioners advocated for the use of psychoanalysis to treat individuals with major mental illnesses including schizophrenia. These practitioners believed that severe mental health challenges were connected in a meaningful way to the life history and self-concept of the individual. Psychotherapy was seen as a means of helping individuals to develop a healthier sense of self and understanding of their own behaviors and emotional states. Psychoanalysis with such individuals was widely practiced into the 1940s. The range of psychotherapy approaches were based on the idea that severe mental illness was primarily caused by pathological family dynamics. As research on genetic influences progressed, psychotherapy fell into disfavor. Further, the focus on family pathology proved alienating to potential family caregivers. Family members favored an understanding of mental illness as genetically and biologically based (Lysaker \& Silverstein, 2009; Swarbrick, 2009).

The 1940s and 1950s saw the introduction of new psychotropic medications and a $280 \%$ increase in per capita expenditures for individuals in state hospitals (Grob, 1994). The availability of psychotropic medications that could reduce psychiatric symptoms created optimism that individuals would be able to function in the community. Deinstitutionalization began in the mid-1950s and there were consistent annual declines 
of psychiatric hospitalizations (Kemp, 2007). As psychiatric medications became more widely prescribed, controversy developed due to adverse effects and misuse (Hardin, Padron, \& Manderscheid, 2014). The anti-psychiatry school of thought arose at this time based largely on the writings of Ronald Laing (1960) and Thomas Szasz (1974). Antipsychiatry maintained that mental illness did not exist but was simply a form of social control (Kemp, 2007). The 1960s were a time of agitation for change and state hospitals became regarded as warehouses of individuals who were being denied their civil rights (Luchins, 2011). With some leadership from President Kennedy whose close family member had been institutionalized, the federal government began providing more money for community mental health centers (Durham, 1989). In the 1970s, states began to revise involuntary civil commitment laws and the number of individuals entering state hospitals began to decrease. Large numbers of people with mental illnesses were discharged from institutions and began to seek lives in the community (Grob, 1994). Unfortunately, funding for community mental health centers proved insufficient and the population of homeless mentally ill people grew rapidly (Manderscheid et al., 2010).

The 1970s witnessed the emergence of the woman's movement, the Black liberation movement, the gay rights movement, and the disability rights movement. In this context, former mental health patients began to organize and advocate for patient rights and against forced treatment, stigma, and discrimination. The groups promoted peer-run services as alternatives to traditional mental health treatment. In contrast to professional mental health services based on the medical model that focused on pathology and management of symptoms, peer-delivered services were based on the principle that 
individuals who have shared similar experiences can support one another through selfhelp and mutual support. Peer services were concerned with building relationships and helping individuals pursue their personal and social goals. The first national annual conference on human rights in mental health care was held in 1972 (Bluebird, 2014). The growth of the movement was supported in part by a growing body of evidence on the limits and serious side effects of psychotropic medications. Antidepressants and antipsychotic medications were found to lead to weight gain, metabolic issues including type two diabetes, neurologic disorders, heart disease, and sexual dysfunction (Joseph, 2013).

The 1980s were a time of transition in which the federal government began providing funds for alternative programs. In the 1980s and 1990s, the effort became known as the consumer/survivor movement. In the 2000s, peer specialists were being trained in all parts of the country. The recovery model emerged from the movement and was focused on promoting hope, self-determination, and community integration (Hardin, Padron, \& Manderscheid, 2014). Hope for recovery was supported empirically by such studies as those conducted by the World Health Organization (WHO) which provided evidence that a remarkable percentage of individuals diagnosed with the most debilitating mental health conditions could realize recovery (de Girolamo, 1996).

\section{Focus of the Study}

The mental health recovery movement that developed in the midst of deinstitutionalization and the civil rights movement emphasized citizenship and social inclusion. However, the term "recovery" has remained ambiguous. While advocates, 
scholars, and government organizations have posited different definitions of recovery, no consensus on a definition or framework exists. It has been conceptualized as a process and an outcome, as symptomatic resolution and functional improvement, and as a personal journey. Despite this lack of clarity, recovery is the mandated aim of the US mental health system (Davidson \& Roe, 2007; Vandekinderen, Roets, Roose, \& Van Hove, 2012). Many believe that core components of recovery include gaining hope, managing symptoms, becoming empowered, and exercising citizenship (Swarbrick, 2009). One definition in wide use was produced by the Substance Abuse and Mental Health Services Administration. It reads:

Mental health recovery is a journey of healing and transformation enabling a person with a mental health problem to live a meaningful life in a community of his or her choice while striving to achieve his or her full potential (SAMHSA, 2004, p.2).

Psychiatric disability and recovery have been explored as inherently social processes. Such exploration requires the measurement of the social aspects of recovery. A review of the literature found no existing measure specific to social recovery. Both an individual's mental distress and recovery take place in social context. Social recovery can be defined as the ability to lead a full and contributing life as an active citizen. Social recovery refers to social interaction, meaningful activity, and community participation (Jaeger \& Hoff, 2012).

Recovery science will only advance with reliable measurement. Psychometrically sound measures are needed to further the empirical work on recovery. The purpose of this study is to develop a measure of social recovery and establish the psychometric 
properties as related to reliability and validity. The study will focus on developing an instrument for use with adults aged eighteen and over.

\section{Personal Relevancy}

Recovery concerns me both professionally and personally. I worked in community mental health in a number of settings for many years before developing a research focus. My family history includes mental illness and substance abuse that resulted in both disability and recovery. Further, I identify as in recovery from bipolar disorder and have found much support from the consumer movement. The curiosity that fuels my research is derived from personal, direct service, and academic experiences.

\section{Relevance to Social Work}

The mission of social work concerns social justice, empowerment, and commitment to marginalized populations. Attention to mental health recovery is consistent with the social work principles of equal rights and opportunities, self-determination, and social justice. Anti-oppressive practice recognizes the role of structural and systemic barriers in shaping the lives of social groups (Morrow \& Weisser, 2012). Social work can be said to be an inherently political practice. Recovery requires the right to participate as a full citizen in society. It is reliant on an enabling social environment that provides opportunities and resources for engagement in relationships and social roles that lead to meaningful inclusion in the larger society (Onken, Craig, Ridgway, Ralph, \& Cook, 2007). Disability theorists have emphasized that a disabling society is itself disabled due to the loss of potential of individuals living with impairment or difference (Bolt, 2005). 
The recovery and social inclusion of individuals with psychiatric disabilities would enrich society and are consistent with the underlying philosophy of social work.

Chapter 2 reviews disability and mad theoretical frameworks and recovery concepts and definitions. The key recovery processes that have been identified as measurable dimensions of change are presented. Literature on recovery outcomes and related social determinants, as well as literature on social capital and inclusion, are explored. The literature pertains to recovery from serious mental illness including those studies that feature psychosis. Attention is given to the importance of consumer involvement in research. Existing recovery measures are reviewed and critiqued. The chapter concludes with a brief justification of the development of a measure of Social Recovery.

Chapter 3 focuses on methodology for instrument development. I present the results of my qualitative study on social recovery processes. The study was used to develop the items for the draft of a social recovery measure. The items are compared to key recovery processes. Items were refined through a process in which they were reviewed by an expert panel and consumers who engaged in a focus group and cognitive interviews. The chapter reviews the instrument development process, methodological rationale, procedures, statistical analyses used to develop and test the measure, and general guidelines of implementation.

Chapters 4 presented the results of the measurement development and validation study. The final version of the Social Recovery Measure (SRM) is introduced. Results of reliability and convergent construct validity testing are reviewed. Chapter 5 summarizes 
the study and discusses the utility of the SRM for direct practice, program evaluation, research, and theory development. 


\section{Chapter 2}

\section{REVIEW OF A THEORY BASE FOR AND THE LITERATURE ON RECOVERY}

This chapter presents disability and mad theories as related to the social exclusion of individuals who experience psychiatric disability. Concepts of recovery are then reviewed. Sections that follow explore recovery outcomes with a focus on social determinants and review social capital and social inclusion concepts and evidence. The emerging literature on the benefits and risks of involvement of consumers in research is presented. The final section of the chapter provides a review of recovery instruments and justification for the development of the Social Recovery Measure.

Theory

Social work has been largely informed by the discourse on disability grounded in the medical model. This model locates the problems of disability within the individual as the genesis of mental illness is ascribed primarily to neurobiological causes (Hamer, Finlayson, \& Warren, 2013). The model assumes deficit and permanent vulnerability of the individual expressed as functional difficulties and symptoms (Ramon, Healy, \& Renouf, 2007). The medical model discourse holds that recovery concerns symptom reduction and maintenance. The relation of symptoms to the life history of the individual and the meaning made of distressing experiences are not considered (Adame \& Knudson, 2007). The dominant stance on psychiatric disability has framed issues in terms of inherent deficit, incompetence, invalidity, and unreliability (Phillips, 2006). Functional limitations and psychological losses are seen as natural consequences for the individuals with impairments. Under this framework, the individual is in need of medical fixing or 
cure. Disability is framed as a diminished state of human existence (Harpur, 2012; Hiranandani, 2005).

In contrast to the medical model, an affirming stance on disability that is emerging views the experience as part of a positive social identity rather than as a personal tragedy. Disability is recognized as a form of human diversity rather than as a condition that needs to be cured or eliminated (Darling \& Heckert, 2010). Disability can be a positive source of personal and political identity instead of a defect or deficiency. Many individuals who live with a disability view themselves as part of a minority group. Such a frame on disability maintains that discrimination and environmental barriers are the principal problems encountered by people with disabilities, not physical impairments or functional limitations. Disability is about social exclusion. Limitations experienced by people with disabilities have social origins and constitute a form of social oppression called ableism or disablism. As a social phenomenon, ableism or disablism can be removed through social change (Hahn \& Belt, 2004; Thomas, 2006). Sanism is the form of ableism concerned with discrimination against people diagnosed with mental health conditions. As a form of oppression, sanism is also the social exclusion of those who cannot conform to social standards of rational thinking and behavior (Morrow \& Weisser, 2012).

Social constructionist and critical theoretical stances on disability maintain that notions of individual inadequacy are socially produced. While disability could be viewed as part of the natural order, it is actually a product of social relations and ways of thinking. Even the most objective of conditions such as visual impairment cannot exist outside of a societal context. Social constructionism maintains that knowledge is a social 
creation situated within values, assumptions, norms, and language. The stance calls for awareness of the effects of social structures constructed by the able bodied. Disability becomes reconceived as a political issue in the same way as other issues such as class, race, gender, and sexuality (Hiranandani, 2005 Shakespeare, 2008).

Locating the problem faced by individuals with disabilities not in disability but in ableism is one means of changing the conversation and challenging the prevailing power structures. Ableism can be seen to operate as a discourse of power. It is sustained through language or rhetoric. Language is the primary means of deriving and communicating the meaning we make of experiences. We understand the world through language. Accordingly, the use of language can also be one of the strategies for struggle against oppression. Language influences how people think and is a powerful tool for influencing how disability is constructed. Any challenge to ableism must involve new language to communicate experiences. Use of words such as ableism and sanism is one such challenge (Cherney, 2011; Harpur, 2012). Individuals with mental health challenges are stereotypically understood as incapable of rational communication. What they say may be reflexively examined through a diagnostic frame and scrutinized for signs or symptoms of mental illness. While individuals may be speaking about their experiences and material and social needs, the content of their speech may be dismissed as evidence of illness (Wolframe, 2013).

The 1970s saw the growth of a disability social movement which defined disability as a social construction. The movement developed out of the disability arts culture which challenged the meanings given to disability in the dominant culture by producing new 
images reflective of the experiences of individuals living with disabilities. Disability arts critiqued the prevailing cultural representations and celebrated disability as an issue of diversity. Art was one way of more positively representing disability as a difference and as a positive source of identity. Deaf theater significantly contributed to providing a prideful presentation of a form of difference. Oppressed groups must define their identity and present a positive sense of group difference in order to assert full cultural citizenship. Culture can be a site of resistance and change (Dupre, 2012; Padden, 2005).

\section{Disability Studies}

The social model of disability first emerged largely in the United Kingdom. The movement emphasized the disabling effects of cultural, political, and environmental barriers. The problem of disability was defined as the failure of society to ensure that the needs of people with disabilities were taken into account when organizing social systems. Barriers faced by individuals with disabilities were explored as the consequence of impairments experienced under social and economic structures (Hiranandani, 2005). The disability rights movement gave rise to the academic field of disability studies. Accordingly, a defining feature of disability studies is that it is both an academic field and an area of political struggle. The disability studies perspective maintains that disability is a complex, interpretative issue that resides in the relationships between people and should be studied as such. The perspective assumes that bodies and minds always appear in the midst of others and so are socially organized experiences. Interrelatedness is an escapable feature of human reality (Titchkosky \& Aubrecht, 2009). 


\section{Deaf Studies}

Deaf studies began to emerge in the 1970s with the assertion that individuals using sign language constituted a cultural group (Humphries, 2014). Deaf studies and disability studies share in common a critique of the ableist paradigm and medical model which are based on deficit and locate problems within individuals. Deafness is identified as an aspect of human diversity (Jordan, 2005; Senghas \& Monaghan, 2002). However, deaf

and disability studies have largely developed separately. Children who were deaf lived in specially designed institutions for generations and were only moved into public schools in the 1970s. The history of institutionalization from childhood contributed to the development of separate deaf cultural groups. The segregated past shaped the social history of the culture and how people with deafness identify themselves. Further, many individuals with deafness identify as a linguistic minority and not as disabled. Deaf study scholars maintain that both the shared and separate perspectives of deaf and disability studies are needed to make sense of the issues of difference and society (Kudlick, 2003; Padden, 2005).

\section{Embodiment}

What type of body can be regarded as legitimate is at the core of disability dialogue. Ableism maintains a bodily standard by which many are judged defective (Loja, Costa, Hughes, \& Menezes, 2013). Feminist theories maintain that while the embodied experiences of disability and gender are material realities, they are overlaid with social meanings and interpretations. Both female and disabled bodies are viewed as inferior or deviant and excluded from full social participation. Feminist disability theorists assert 
that in addition to the social and political aspects of disability, the personal experience of living with impairment and disability needs to be acknowledged. Identity is fundamentally an embodied experience. This school of thought emphasizes that the personal and the political realities are connected (Hiranandani, 2005; Loja, Costa, Hughes, \& Menezes, 2013).

To appreciate experiences with disability, attention must be paid to biological or embodied realities. Denying the role of biology in disability would deny lived experience. One's experience with embodiment results from a complex relationship between corporeality and society. People with disabilities build subjectivity through physical interactions with the social environment. Exclusion of the body can lead to a particular type of philosophical reductionism that does not account for lived experience (Anastasiou \& Kauffman, 2013; Hughes, 2007).

There has been little effort to apply concepts regarding embodied impairment to psychiatric disability. However, the concept of embodiment can act to dissolve the mind/body distinction and can allow for understanding lived experiences as both biologically and socially produced. Mulvany (2000) coined the term embodied irrationality. She asserts that a focus on embodied irrationality would encourage the study of how individuals make sense of bodily experiences of mental distress while avoiding biological determinism. The concept would allow for a focus on the complex relationship between the biological and the social factors of madness. Mulvany maintains that the study of psychiatric disability should include analysis of embodied irrationality and disabling environments. 


\section{Intersectionality}

Intersectionality theory emerged from feminist thought. Intersectionality can be used to examine the ways in which such social and cultural categories as race, gender, sexual orientation, class, and disability operate together to shape identity, experience, and power relations on both individual and social institutional levels. The theory recognizes that it is not possible to separate out the social categories or explain inequalities by focusing on one without the others. Identities can be multiple and can be defined and experienced through one another (Pilling, 2013). Social power is differentially distributed through a hierarchy or matrix of social identities. Systems of oppression and privilege interlock with one another and may compound experiences of marginalization. The theory can be used to explore the social and political processes that create privilege and oppression. Experiences of mental distress take place in a social context that values some individuals over others. Psychiatric disability cannot be understood on its own or isolated from other social identities. Some mental illness symptoms and conditions are linked to conditions of poverty and other forms of social inequity (Morrow \& Weisser, 2012).

Disablism intersects with sexism, racism, homophobia, ageism, and socioeconomic stratification such that forms of disadvantage and many strands of identity are formed (Thomas, 2006). Some have written that it can be difficult for someone living with multiple intersecting oppressions to distinguish instances of sanism from other types of discrimination given how closely bound are the forms of oppression. Intersectionality also implies that individuals with lived experience of madness must contend with the 
differences and inequalities among them so as to best support relationship and community building (Wolframe, 2013).

\section{Anthropological Thought}

Some theorists, most particularly those working in anthropology, have emphasized that able- bodied individuals struggle with disability as they do not want to be reminded of their own vulnerabilities. The disabled body exposes the illusion of absolute autonomy that is the basis of a fantasy of complete able-bodiedness and independence (Hughes, 2008). Unlike such categories as race and gender, disability as a social category is distinct as anyone may enter it at any time due to unforeseen circumstances, injury, or aging. As such, disability challenges ideas about lifelong stability of normativity and identity. No other social category can be free from the possibility of disabling experiences. This form of difference can be found across human experiences and cultures. At the same time, not all cultures recognize the disabled as a social category. For instance, psychiatric conditions can be seen as divine gifts or as curses depending on the culture and point in history (Ginsburg \& Rapp, 2013). Through study of the diversity of cultural stances on disability, anthropology can help denaturalize the relationship between difference and social exclusion and discrimination. The discipline maintains that one's capacity to cope with conditions is determined by the availability of material and social resources. What conditions are regarded as problematic is held to be socially constructed (Battles, 2011).

Another contribution of anthropological thought concerns the exploration of spirituality and disability. Compared to the majority population in Western cultures, nonWestern and aboriginal conceptions of disability are more likely to include a focus on 
spirituality and on collective as opposed to individual experiences. Embodiment of experience can be not only an individual matter but also an issue for the collective in cultures that value inter-dependence over independence. Disability can be understood in the context of community and what the disability experience either requires from, or provides to, the community. One aspect of spirituality is the interconnectedness of a people. Inclusion of spirituality may act to value or incorporate non-rational experiences (Stienstra \& Ashcroft, 2010).

\section{Queer and Crip Theories}

Queer theory views identity as fluid and as subject to ongoing social construction. What is viewed as normal versus queer may change. Similarly, the category of the disabled cannot be fixed as it depends on environments that enable or disable (Sherry, 2010). The world does not consist of normal people and the mentally ill, but of individuals who may experience mental distress at some point in their lives (Wolframe, 2013). The field of disability studies has long focused on how a society incorporates some bodies but not others.

Both disability studies and queer theory challenge societal processes that marginalize those who don't conform to standards of normalcy. Both fields of study are grounded in the feminist deconstruction of essentialist categories of identity. Identities are not natural but socially subscribed and shifting. Feminist, disability, and queer theories view dichotomous approaches to identity as illegitimate and wounding, as there are many ways of being a human. 
Disability studies has been queered and developed into Crip theory. Crip theory has explored how disability and desire are often placed at odds. That is, disability is framed as undesirable and disabled individuals are often viewed as asexual. This view further devalues the disabled individual (McRuer, 2010). Sexual expression among individuals with disabilities has been denied in part due to fear of their reproduction (Ginsburg \& Rapp, 2013).

\section{The Diversification of Disability Theory}

Disability studies is a multidisciplinary field and it is clear that disability theory has experienced significant eclecticism or diversification. The points of agreement among disability theorists consist of the need to avoid biological reductionism and to maintain a social relational understanding of disability (Peters, Gabel, \& Symeonidou, 2009; Thomas, 2006). The construction of difference as an obstacle to belonging is a social phenomenon (Titchkosky \& Aubrecht, 2009). Disability theory developed as resistance to the medical model of disability, oppression, and ableism. The theme of resistance is woven throughout disability studies. It could be said that resistance is the theoretical bridge in the diverse field of disability studies. Resistance exists on a continuum from the individual to the collective and is concerned with power and strategy. If the commonality of resistance is recognized, diverse stakeholders can strategize and act jointly towards political ends. Resistance can support community building and taking steps towards changing a disabling society (Gabel \& Peters, 2004).

Ableism imposes a corporeal standard. One means of destabilizing ableism is through the development of a politics of difference. The politics arises from resistance to ableism 
and from valuing human variation through recognition of the equal value of different ways of being. Differences and impairments are part of lived experience and cannot be completely separated from identities. Identity is fundamentally embodied and influenced by subjective bodily experiences and social encounters. A new politics is required in order to recognize and respect the valued aspects of the disabled identity. A disability politics will be in part a politics of embodiment (Loja, Costa, Hughes, \& Menezes, 2013; McRuer, 2010).

\section{Internalized Oppression}

There is a body of literature that explores how a hierarchy of impairment appears to exist among people with disabilities. Some individuals with disabilities hold views that devalue others in different subgroups. The logical outcome of people with disabilities stigmatizing other people with disabilities is the furtherance of social oppression of the entire disabled population. One disabled researcher who explored these dynamics explains that the basic desire of a person for a positive self-concept may lead to distancing from others lower down in a hierarchy so as to reduce the stigma of association. It is therefore essential that the problem become framed not as disability but as one of representation and discrimination (Deal, 2010). Critical race theory has been applied to this issue of internalized ableism. Just as racism is not aberrant but the current order of social life, ableism permeates our culture. The belief in the inherent negativity of disability is internalized by those with and without disabilities. Internalized ableism results in self-hate and the distancing from and hatred of others in one's group. This perspective helps elucidate the need to build community and collective consciousness in 
order to develop more supportive perspectives on disability experiences (Campbell, 2008).

In the case of individuals with lived experiences of mental health challenges, the only frame of reference they may have available is the predominant medical model which has a lens of pathology and places individuals in diagnostic categories. Unless such individuals have access to other ways of making meaning of lived experience, they may discount themselves and others with similar experiences (Beresford, 2005). Individual and political identities exist in relation to one another. A positive self-identity as a disabled person involves building critical consciousness and is a prerequisite for collective political action. Resistance begins with individual and collective recognition of oppression. True social power will only be constructed through adoption of disability as a positive identity and practice of collective resistance (Peters, Gabel, \& Symeonidou, 2009).

\section{Mad Studies}

While psychiatric disability has been a rare focus of disability theorists, the field of mad studies is developing out of the foundation of disability studies. Development of the discipline is more active in Canada and the United Kingdom compared to the USA. The emphasis on self-determination is a commonality between the disability and mad social movements (Jones \& Brown, 2013). The notion that psychiatric disability includes embodied experiences and interactions in social environments provides a framework for studying the impact of social phenomena on individuals with lived experience of madness (Mulvany, 2000). Mad studies aims to explore how experiences and needs may be 
communicated through metaphors and other forms of expression that may be otherwise labeled as symptoms. Expression of mental distress is viewed as meaningful and as connected to interpersonal and social experiences. Mental diversity is valued as part of the human experience. Application of disability theory frameworks to mental health issues highlights experiences of oppression and discrimination as they relate to distress and recovery. Disability theory can help shift the focus away from individual pathology to the effects of social exclusion of individuals who have experienced madness (Beresford, 2005).

The focus on institutionalized oppression would help identify the social barriers that restrict access for individuals with psychiatric disabilities to the rights of full citizenship. Mental health policy rests on the assumption that problems faced by those with psychiatric disabilities result from their illness. The broader social factors of poverty, discrimination, and social exclusion are not targeted (Mulvany, 2000). Mental illness can be alternatively framed as attesting to the profoundly damaging impact of social adversity and inequality through the powerful biological impact on the individual (Barnes, 2011).

Disability and mad theories maintain that disability is not located simply within the body but is created by social conditions that act to disable the full participation of individuals with a variety of physical and mental states of being (Ginsberg \& Rapp, 2013). The premise of disability studies is that the meanings and practical impact of disorders result from cultural responses to perceived differences. Disability can be seen as deprivation as it interferes with a person's ability to make valued choices and participate fully in society (Hopper, 2007). The theories maintain that society must 
become more accepting of the full expression of the human experience so as to value mental diversity (Morrow \& Weisser, 2012).

\section{Social Work}

While just reviewed, the precepts of disability and mad theory will next be applied to social work as they are congruent with the discipline's mission of social justice, empowerment, and commitment to marginalized populations. The theoretical studies and associated civil rights movements share with social work the principles of equal rights and opportunities, self-determination, and social justice. Anti-oppressive practice emerged from social work and recognizes the role of structural and systemic barriers in shaping the lives of social groups (Morrow \& Weisser, 2012). The stance that degrees of disability are determined by transactions of the person and the environment is consistent with the core of the social science of social work. Disability is a relationship or interaction of the person and the environment. The core of disability and mad thought is the belief that society must change, not the individual. The "mad pride" agenda asserts the rights of people who have experienced madness and advocates for changing the social world through respecting, responding, and incorporating difference so that the world is a fit place for them to live (Costa et al., 2012). In place of an emphasis on individual rehabilitation or adaptation, the environment becomes the target of intervention. Framing disability as a social reality determined as much by structures and resources as by any underlying impairment demands a focus on intervening to support social inclusion instead of focusing on only providing treatment (Hopper, 2007). This is not to deny the role of biology, but instead is to recognize that the biological occurs in a social context 
that involves intersecting forms of oppression that impact mental health (Morrow \& Weisser, 2012).

A human rights stance would maintain that society must accord individuals with disabilities the rights and responsibilities of citizenship without the contingency of first overcoming disability. An individual with a psychiatric disability needs to be able to exercise self-determination and have a meaningful life while continuing to have the psychiatric disability (Davidson \& Roe, 2007). Recovery requires the right to participate as an equal citizen. Citizenship should not depend on reaching normality but should rest on inclusive communities. Inclusion is not about changing people but changing society (Slade, Amering, Farkas, Hamilton, O’Hagan, Panther, Perkins, Shepherd, Tse, \& Whitley, 2014). Social work's necessary task would concern the expansion of real opportunities (Hopper, 2007).

While there is little social work literature related to disability and mad culture and theory, some have called on the discipline to collaborate with people with disabilities and activists in order to re-narrate disability as part of the human experience (Beresford, 2005; Dupre, 2012). Mad-identified individuals have organized for advocacy and have held pride events that celebrate difference, challenge prevailing social narratives, and allow socially-excluded individuals to redefine the disability experience (Schrader, Jones, $\&$ Shattell, 2013). Study of the culture and theory is one avenue for examining structural oppression and developing a politics of difference (Dupre, 2012). This politics requires an alliance with social science and academia (Shakespeare, 2008) and concerns an examination of the processes that produce difference and exclusion (Battles, 2011). 
Social work should challenge the social systems that discriminate against individuals with lived experience of distress and madness (Beresford, 2005; Harpur, 2012). Adoption of the stance that people with disabilities are not lesser individuals in terms of moral worth or human rights necessitates a struggle for a more inclusive definition of citizenship which incorporates individuals of non-normative mental experiences (Ginsburg \& Rapp, 2013; Shakespeare, 2008). If social work were to frame practice as a matter of promoting citizenship of service users, the political power of social work would be engaged in support of full recovery journeys (Hamer, Finlayson, \& Warren, 2013). The work involves rethinking what is possible for individuals with lived experience of madness and raising expectations for social connectedness and citizenship (Ware, Hopper, Tugenberg, Dickey, \& Fisher, 2007). Recovery relies on a social environment that provides opportunities and resources for engagement in relationships and social roles and for meaningful inclusion into the larger society (Onken, Craig, Ridgway, Ralph, \& Cook, 2007).

Like all social sciences, the discipline of social work has involved the measurement of social phenomena. Development of a measurement instrument that emphasizes a positive self-concept, self-determination, and social connectedness would be consistent with the precepts of disability and mad theories. Further, it would be necessary to develop the measure in partnership with individuals in recovery from mental health challenges. Recovery concepts and outcomes will be explored before returning to consumer involvement in research and measure development. 


\section{Recovery Concepts}

The recovery movement developed in the 1960s and 1970s during deinstitutionalization, the growth of self-help groups, and the civil rights movements. Ideas that promoted a life in the community with adequate care and support were developed during this period (Vandekinderen, Roets, Roose, \& Van Hove, 2012). The consumer/survivor/ex-patient movement of the time conceived of mental illness as a complex phenomenon that was an emotional, behavioral, biological, and spiritual manifestation of the interplay of social, emotional, and cultural stressors. Mental illness was framed as reflective of crises as opposed to physical disease (Cohen, 2005). Members of the movement claimed that individuals who had experienced what was generally understood to be mental illness had special insight into the experiences and should be able to speak on their own behalf. They adopted a firm stance that their perspectives represented real knowledge that should be incorporated into public decision making (Costa et al., 2012). Members celebrated "mad pride" and contended that the route to healing involved acceptance of mental diversity by society. The Oregon Insane Liberation Front was founded in 1970 as the first consumer-run rights group and was followed by other Mental Patients' Liberation Movement groups in New York, Boston, and San Francisco (Ostrow \& Adams, 2012; Tomes, 2006). The patients' movement stood in opposition to the medical model and in support of self-reliance and selfdetermination. The various groups provided advocacy and self-help alternatives to the psychiatric system. The basic liberation principle was that people must speak for themselves (Chamberlin, 1990). While these concepts developed almost half a century 
ago, the majority of the peer reviewed literature on mental health recovery has been published since 2007 and only a minority of it has been produced by individuals with lived experience of mental distress and recovery (Stickley \& Wright, 2011).

The term "recovery" is ambiguous. Neither a concise definition nor consensus on a conceptual framework exists. The only consensus in the peer reviewed literature is said to be that there is no consensus on the definition of recovery and that conceptual confusion is great (Forrest, 2014). Recovery can be seen as both a process and an outcome and as having both objective and subjective indicators. It has been conceptualized as symptomatic resolution, as functional improvement such as vocational or educational involvement, as a personal journey, and as a social process. The lack of consensus on how to define recovery can be seen as a reflection of the lack of consensus regarding the definition of mental illness. This ambiguity complicates the mental health field and research (Onken, Craig, Ridgway, Ralph, \& Cook, 2007).

Recovery as a relative concept appears to mean different things to different individuals with lived experience of mental illness. However, the core ideas identified in the consumer literature include recovery of lost potential and regaining some degree of control over one's personal and social life (Ramon, Healy, \& Renouf, 2007). Consumer definitions have been found to concern strengthening of the self-concept, rebuilding a life through active social engagement, and having hope for one's future after experiences with emotional distress (Pitt, Kilbride, Nothard, Welford, \& Morrison, 2007). Current definitions are limited as they have been determined consensually rather than empirically. As recovery is not defined by particular practices or services, it may represent a vague 
philosophy or value orientation to care (Bellack, 2006; Braslow, 2013; Smith-Merry, Freeman, \& Sturdy, 2011). Despite the lack of a clear understanding of recovery and confusion regarding what would be entailed in transforming mental health services in order to promote it, recovery is the mandated aim of US mental health policies and services (Davidson \& Roe, 2007; Jaeger \& Hoff, 2012).

One emerging differentiation in recovery is between clinical recovery which involves a return to normalcy and personal recovery which is an individually directed process (Slade et al., 2012). Individuals with lived experience of mental distress or madness and those researchers who have been influenced by them make the distinction between (a) recovery from symptoms and related deficits, and (b) the recovery of dignity, autonomy, and self-respect whatever the symptomatic course. Distress caused by mental illness is greater than symptoms alone given the associated loss of social roles. Individuals in recovery need to reestablish their lives and access social and material resources. This definition of recovery is conceptually distinct from a medical definition of remission and concerns rebuilding a worthwhile life inclusive of a positive self-identity and valued social roles (Gordon, 2013; Tew, Ramon, Slade, Bird, Melton, \& Le Boutillier, 2012). Consumers maintain that the goal of the recovery process is not to become normal but to exercise self-determination and reengage in personal development (Barker, 2003). However, biomedical and recovery-oriented services are not inherently opposed and can be complementary or mutually reinforcing. Symptom improvement and recovery of one's life can co-exist and one can support the other (Davidson \& Roe, 2007; Resnick, 
Rosenheck, \& Lehman, 2004). An individual can also experience remission of symptoms without experiencing personal recovery (Slade et al., 2014).

A systematic review and narrative synthesis of the conceptual frameworks of recovery posited that recovery processes can be understood as measurable dimensions of change or outcomes (Leamy, Bird, Le Boutillier, Williams, \& Slade, 2011). There are four aspects of personal recovery for which consensus has emerged from qualitative studies: hope, self-identity, meaning in life, and personal responsibility (Jaeger \& Hoff, 2012). A significant distinction can be made between recovering from mental illness and being in recovery. While recovery "from" concerns remission and objective functional improvement, being in recovery concerns how an individual manages his or her life with an enduring illness. Being in recovery refers to a unique process rather than a set outcome. It involves self-determined pursuits and leading a meaningful life and is reflective of one's social and political experiences (Davidson, Schmutte, Dinzeo, \& Andres-Hyman, 2008).

A sole focus on the personal and unique aspects of the recovery process can act to obscure the fact that recovery takes place in a social context. The processes involved in individual recovery can be readily linked to the social aspects of experience that can either facilitate or impede inclusion in community life. The social nature of recovery can be explored in terms of social interaction. An individual recovers in the context of relationships and a social environment (Tew et al., 2012; Topor, Borg, Girolamo, \& Davidson, 2011). Those who strongly associate social inclusion with recovery define it in contrast to the illness experience which involved loss of social roles and meaningful 
activity. Recovery involves overcoming the effects of rejection by family and friends, social exclusion, and loss of the sense of self as an effective social agent (Bromley et al., 2013; Stickley \& Wright, 2011). Those who define recovery as social integration assert that recovery involves consideration of not only individual quality of life but a focus on required social change (Ware, Hopper, Tugenberg, Dickey, \& Fisher, 2007). A view of recovery that emphasizes the need for social change as opposed to individual change is linked closely with a human rights and citizenship focus (Frese, Knight, \& Saks, 2009).

\section{Social Recovery}

Clinical stances on recovery have dominated the field and have led to an underemphasis on the importance of the wider environmental context. An ecological framework would include characteristics of the individual, social factors, and the interaction of the two (Leamy, Bird, Le Boutillier, Williams, \& Slade, 2011). Mental illness is becoming understood as intrinsically social (Morgan, Burns, Fitzpatrick, Pinfold, \& Priebe, 2007). The most basic definition of social recovery is the ability to lead a full and contributing life as an active citizen. This model views recovery as establishing a satisfying and meaningful life with an impairment rather than waiting to be without it. The recovery process involves introduction of the individual into an accepting environment. The core narrative of recovery concerns moving from chronic disability to a dynamic life (Secker, Membrey, Grove, \& Seebohm, 2010). Social recovery refers to experiencing meaningful relationships, a sense of being able to make a social contribution, and a sense of belonging (Slade, 2010; Whitley \& Drake, 2010). The dimensions of recovery clearly overlap as resolution of internal distress can take place 
alongside of social reengagement in ways that are mutually reinforcing (Jaeger \& Hoff, 2012; Tew, 2012). However, longitudinal studies have found that symptom improvement and relapse prevention are not necessarily linked to pursuit of education, employment, and social relationships. Such outcomes may require their own assessment (Priebe, 2007). It is safe to say that recovery is multidimensional with aspects in complex relationship (Norman, Windell, Lynch, \& Manchanda, 2013).

Social disability is defined as difficulty performing family, social, and occupational roles expected of an individual by the group or community to which he or she belongs. Problems related to social disability of individuals with mental health conditions have become a focus in the recovery field more recently than the focus on clinical recovery which involves symptom improvement (Bottlender, Straub, \& Moller, 2010). In addition to not being as well researched as symptomatic recovery, social recovery has only been identified as a target area for mental health policy within the last decade (Hodegekins, 2012). The majority of recovery-oriented research emphasizes that recovery involves development of a coherent sense of self and personal responsibility for social reintegration. This framework becomes problematic given the lack of available choices and resources afforded to some members of society. An individual approach to recovery does not take into full consideration the structural inequalities that can hamper personal and social pursuits. Individuals cannot be asked to assume responsibility for social inequities, and problems must be viewed in their political and economic context. A focus on characteristics of people with mental health issues can obscure the policy and system 
issues that are needed to best support them (Vandekinderen, Roets, Roose, \& Van Hove, 2012).

The underexplored area of social recovery concerns the effects of social expectations or demands on the individual with lived experience of mental health challenges. Social demands coupled with accommodations or supports have been said to enable recovery. Social recovery involves social interaction, communication, community participation, citizenship, economic stability, and wellness (Jaaskelainen et al., 2012). It involves leading a valued life irrespective of impairment or difference. Beresford (2002), a leading theorist, has asserted that social recovery involves renewing a sense of possibility, regaining competencies, reconnecting and finding a place in society, and reconciling illness and disability experiences with a positive sense of self. Social equality more than healing is the dominant focus. Under this framework desired outcomes of interventions include the enhanced agency that is required for genuine social participation. Recovery on the part of individuals becomes dependent on a society that supports their social inclusion. Social recovery requires the development of social environments that are both accepting and enabling (Beresford, 2002; Hopper, 2007).

Included in the social recovery framework is the concept of relational and inclusive citizenship. As opposed to normative citizenship which involves norms of individualism and self-sufficiency and is predicated on meeting normed standards of ability and productivity, relational citizenship is negotiated in social context and maintains that membership and equality of status apply to all citizens. Reintegration into society has 
been recognized as generating feelings of hope (Vandekinderen, Roets, Roose, \& Van Hove, 2012).

Hope

The commonly held view that mental illness is a life-destroying experience communicates hopelessness. As might be expected, hope has been identified as a central component of recovery. The need for hope is a prominent feature of consumer accounts of recovery and is explored at length in the grey literature produced by consumers. Ridgway (2001) analyzed recovery narratives and found that a period of despair often followed diagnoses of psychiatric disorders and that recovery was characterized by a renewed sense of hope. Hope is enhanced by development of the sense that one can control one's life and by seeing how others have moved forward. Hope has been said to contribute towards a positive identity (Perry, Taylor, \& Shaw, 2007; Stickley \& Wright, 2011). A systematic review of the international literature on recovery conceptual frameworks found that hope was defined as a vital component of the recovery process as it contributes to the development of a valued self, meaning in the illness experience, and life in general (Slade et al., 2012).

A systematic literature review of definitions and measurement tools of hope found that the concept had a number of dimensions. These dimensions were summarized as a futureorientated expectation of attaining valued goals which would provide meaning, are considered possible, and depend on personal activity or characteristics and/or external social and material resources. Hope was consistently positively associated with perceived recovery, self-efficacy, self-esteem, empowerment, spirituality, quality of life, social 
support, and service availability. There were no studies available that investigated the direction of effects. However, individuals in treatment for first-episode psychosis have reported that hope was essential for recovery. The availability of role models who were in recovery inspired hope that was essential for their own recovery efforts. Messages of hope appeared to buffer stigma and contribute to the development of a competent sense of self (Romano, McCay, Goering, Boydell, \& Zipursky, 2010; Windell \& Norman, 2012). Individuals have stressed the importance of belonging to social groups for feeling hopeful. Being alone or excluded led to hopelessness. In this formulation, hope is essentially an interpersonal phenomenon (Perry, Taylor, \& Shaw, 2007).

\section{Cultural and Diversity Issues}

The main finding from a systematic review of international differences in recovery frameworks was that current conceptualizations are primarily based on Western European and North American models. The authors concluded that the approach to recovery seemed to have taken an individualizing or personalizing direction and to have undervalued the strength that people can gain from one another and in community (Slade et al., 2012). As such, recovery concepts may not resonate with individuals from minority groups. The importance of self in the recovery process is a very Western concept (Tooth, Kalyanasundaram, Glover, \& Momenzadah, 2003). The value placed on autonomy does not have the same importance across communities and cultural groups. Cultures that value collectivity over individuality or inter-dependence over independence may conceive of recovery as matters of group or cultural processes (Morrow \& Weisser, 2012). Recovery may need to be broadened to include a focus on community and cultural 
resilience and well-being (Slade et al., 2014). Some cultures and traditions may create meaning from illness experiences and establish meaningful social roles through a collective approach (Onken, Craig, Ridgway, Ralph, \& Cook, 2007).

Studies that focus on people of color place greater emphasis on spirituality and stigma as well as on culturally specific factors and collectivist perspectives of recovery (Jaeger $\&$ Hoff, 2012). One thematic analysis of studies of recovery for people of color in the USA found that stigma associated with race, culture, and ethnicity combined with the stigma associated with mental illness. Being an individual from a minority group accentuated the stigma of mental illness as individuals viewed themselves as belonging to multiple disadvantaged groups. They needed to recover from racial discrimination and violence, not just mental illness (Leamy, Bird, Le Boutillier, Williams, \& Slade, 2011). Indigenous recovery may involve the articulation of a shared narrative and identity (Smith-Merry, Freeman, \& Sturdy, 2011). The central recovery component of hope may have different meanings in different cultures and population groups. Indeed, current definitions in the recovery literature do not consider hope at the societal level (Schrank, Bird, Rudnick, \& Slade, 2012).

Despite a diversity of views on recovery, there is agreement that recovery-focused mental health practice is dependent on the life situation and history of the service user (Smith-Merry, Freeman, \& Sturdy, 2011). However, empirical investigation of recovery often fails to take into account structural issues of poverty and other aspects of disenfranchisement. Social forces such as racism, sexism, and heterosexism can hinder recovery while material resources and access to social connections and institutions can 
enable recovery. Without social power and access to material resources, individuals lack the external capacities of valued social roles, strong interpersonal relationships, and community inclusion needed for recovery (Stickley \& Wright, 2011; Hopper, 2007). The ability to exercise individual autonomy may be devastated by the impact of racism and other forms of discrimination (Barnes, 2011). Recovery within diverse populations involves reclaiming culture and community and overcoming the double stigma of mental illness and social standing. Being able to participate fully in society refers to participation as one is, not as society prescribes. An informed recovery would entail appreciation for the effects of an environment featuring racism, sexism, classism, colonization, and homophobia (Ida, 2007).

Little research has been conducted on the perspectives on recovery of LGBTQ individuals. This matter is especially notable as LGBTQ people were once defined as mentally ill based on their sexual and gender identities. The longstanding pathologizing of identity has resulted in a complicated relationship to mental health concepts (Pilling, 2013). LGBTQ individuals also experience the ongoing trauma of discrimination from the wider society because of their sexual orientation (Ida, 2007). A study of LGBTQ women (Das, 2012) found low resonance with recovery concepts. The women reported that they did not think about recovering from experiences with trauma and mental distress as these experiences would always be part of their lives. However, they did describe a need to recover from the mental health system itself and from societal attitudes towards individuals who experience distress. Individuals had experienced that their sexual or gender identities had been labeled mental illness and continued to be concerned that they 
would be pressured to recover from marginalized ways of being in the world. Therefore, the researcher recommended that supporters use the language chosen by the individual to describe experiences such as gender outlaw or queer.

\section{Definition}

The language of recovery is widely used in mental health services and research, yet the term is used in a variety of ways. Broad and multifaceted definitions allow for individualization of support but present a disadvantage at conceptual and implementation levels (Ramon, Healy, \& Renouf, 2007). A systematic review and modified narrative synthesis of the recovery literature was undertaken in order to construct an empiricallybased conceptual framework for recovery (Leamy, Bird, Le Boutillier, Williams, \& Slade, 2011). Key recovery processes were identified and understood to be measurable dimensions of change which typically occur during recovery. The conceptual framework provides a taxonomy of recovery outcomes. These identified processes known by the acronym CHIME are:

Category 1: Connectedness: support, relationships, community involvement Category 2: Hope: belief in recovery, motivation, hope-inspiring relationships, goals Category 3: Identity: positive sense of self and identity, overcoming stigma Category 4: Meaning in life: meaning of mental illness experiences, quality of life, meaningful life and social roles, meaningful life and social goals, rebuilding a life Category 5: Empowerment: personal responsibility, control over life, focus on strengths (p. 448). 
The next section will explore the empirical evidence regarding recovery outcomes. Recovery will be placed in a social context. Attention will be given to first-episode psychosis, the impact of gender and age, and the importance of a sense of self and meaning in life.

\section{Recovery Outcomes}

The concept of recovery developed from emerging research that countered long-held assumptions about the chronic nature of psychiatric disability (Mancini, Hardiman, \& Lawson, 2005). This section will review literature that explores the recovery process and factors that both hinder and support recovery journeys. How social context appears to determine outcomes will be explored.

Schizophrenia and related psychotic disorders are viewed as the most disabling mental health conditions. Reviews of outcome studies have noted great variation of findings across treatment settings and countries. Assessment of social functioning is highly problematic given the wide variation in social norms, expectations, and beliefs. Researchers have emphasized the need for a standard framework for comparing schizophrenia outcomes across social environments. Methodological heterogeneity found in studies may account for some of the variation found in outcomes (Cohen, Patel, Thara, \& Gurje, 2008). A systematic review and meta-analysis of recovery in schizophrenia considered both clinical and social outcomes. Across countries, for every 100 individuals diagnosed with schizophrenia, 1 or 2 individuals a year would meet recovery criteria and $14 \%$ would reach a state of recovery over 10 years. Studies from low-income nations had significantly higher rates of recovery. Findings were consistent with previous systematic 
reviews. The proportion of individuals with schizophrenia and related psychosis who meet criteria for clinical recovery appears not to have increased across time (Jaaskelainen et al., 2012).

Longitudinal studies that focus on recovery as occupation of valued social roles and as community involvement have consistently found that half to two-thirds of individuals with schizophrenia significantly improve (Tooth, Kalyanasundaram, Glover, \& Momenzadah, 2003). The World Health Organization (WHO) has been conducting studies on schizophrenia since 1967 in thirty sites located in nineteen countries in the developed and developing worlds. While incidence has not varied greatly across the locations, outcome has consistently been better in developing countries such as India compared to developed countries such as the United States. The WHO researchers have speculated that the better prognosis in developing countries may be accounted for by role maintenance and community integration (de Girolamo, 1996; Eisbenberg, 1988; Hopper, 1991; Hopper \& Wanderling, 2000).

Individuals with early-onset psychiatric disorders (at or before 18 years of age) are known to have worse social outcomes as compared to those with adult onset. One large study $(N=5,839)$ in the US found that compared to individuals with adult onset, individuals with early-onset psychiatric disorder were more likely to be unemployed, to have less than a high school education, to have a lifetime arrest record, and to have received public assistance. As compared to Whites, Latinos were more likely to be unemployed, fail to complete high school, and to have been arrested, Asians were more likely to be unemployed, and Blacks were more likely to be unemployed, to have less 
than a high school education, to have a lifetime arrest record, and to have received public assistance. Differences in the rates of social disadvantage across racial/ethnic groups mirrored those found in the general US population, except rates were two to six times higher for those with early-onset psychiatric disorders (Le Cook, Carson, \& Alegria, 2010).

\section{First-Episode Psychosis}

Individuals who experience a first episode of psychosis often withdraw socially as a means of coping with overwhelming experiences that are difficult to explain to others. They express a need to develop a personal understanding of the confusion of psychosis (Judge, Estroff, Perkins, \& Penn, 2008). By their report, social support is the most positive influence on recovery. Having individuals on whom they can depend and having a sense of being valued are highly important. Friends without psychiatric disabilities who were part of the pre-illness social network provide a sense of continuity and social inclusion, while friends with psychiatric disabilities provide empathy and companionship. Valued activities provide a sense of meaning and social value. Being in roles in which they could assist others was especially helpful due to the experience of reciprocity and equality (Windell \& Norman, 2012).

The illness experience has been described as including significant stress, reduced activity levels, and life disruption. The recovery process involves understanding the contributors to distress and illness and making commitments to necessary changes in a context of social supports. Some individuals have reported that their sense of self became stronger through the process of learning to manage issues and engage in recovery 
(Romano, McCay, Goering, Boydell, \& Zipursky, 2010). Youth in recovery highly value having a sense of choice and control, and report increased feelings of empowerment under such conditions. They wish to choose which coping strategies to implement and to make their own decisions about their lifestyles. Being listened to was perceived as respectful, supported a sense of empowerment, reduced confusion, and led to feeling understood. An opportunity to talk about symptoms was also empowering as the process increased their understandings of symptoms, reduced anxiety, and facilitated the ability to seek help (Grealish, Tai, Hunter, \& Morrison, 2013).

Trajectories and predictors of social recovery were explored for a sample of 878 individuals involved in an early intervention for psychosis program (Hodgekins, 2012; Hodgekins et al., 2015). Individuals were accessed at program entry and one year later. Almost 17\% of individuals made a partial social recovery and just under a third made a full social recovery. Social disability was predicted by male gender, ethnic minority status, and poor premorbid functioning. A prospective study (Austin et al., 2013) that followed 496 individuals with first-episode psychosis and schizophrenia spectrum diagnoses collected data at baseline, one year, two years, five years, and ten years. Full recovery was defined as the stable remission of symptoms, no psychiatric hospitalizations for the past two years, and engagement in work or education with only moderate difficulty. A total of $14 \%$ met criteria for full recovery at ten year follow up and nearly a third of the entire cohort had achieved full recovery at some point over the ten year period. It appeared that individuals moved in and out of recovery. Greater involvement in education, work, and social pursuits was associated with better recovery. The 
researchers concluded that reintegration of individuals into social roles should be a focus for interventions. Another study of individuals involved in an early intervention program for psychosis focused on social support and recovery. severity (Norman, Windell, Lynch, \& Manchanda, 2013). The study found that the degree to which someone perceives that others regard the relationship as valuable, important, or close was the most consistent and important predictor of subjective recovery. The finding was independent of symptom severity.

An exploration of how ethnicity and culture relate to the self-appraisal of individuals with first-episode psychosis found that Black individuals held less negative appraisals compared to other ethnic groups (Upthegrove, Atulomah, Brunet, \& Chawla, 2012). The association was not related to level of insight or recovery style. Black participants experienced less loss as a result of experiences with psychosis and perceived greater control over their illness. The researchers offered two potential explanations for the findings: (a) Black individuals may attribute illness to external causes as they are more likely to hold social or spiritual explanations for illness, or (b), they may have had lower life expectations prior to the illness and so have experienced less loss. The researchers concluded that the pros and cons of health belief models should be further explored. The study reinforces the significance of social support and inclusion for mental well-being.

\section{Gender Differences}

While epidemiological studies do not find gender differences in the prevalence of schizophrenia, differences in age of onset by gender are the most replicated study finding. Men develop the disorder at age 18-25 while women develop the disorder between the 
ages of 25-35. Most studies find no significant clinical or symptomatic differences between the genders. However, those studies that do find differences note a higher prevalence of negative and disorganization symptoms for men and a higher prevalence of affective symptoms for women. Most studies do find that men have worse premorbid functioning. One possible explanation for this gender difference is the earlier age of onset for men. Women have higher levels of social support and adjustment before the onset of the illness and present with better social functioning and less disability throughout the life course. The higher age of onset for women may allow them to occupy social roles that facilitate ongoing community support. Apparently, the greater social support results in less disability for women (Ochoa, Usall, Cobo, Labad, \& Kulkarni, 2012). In one study of schizophrenia and social functioning, defined as capacity to adjust to personal, family, social, and professional needs and perform in socially defined roles, women had higher levels of social functioning despite having similar symptom severity. The researchers hypothesized that women have better coping strategies and/or make better use of available services (Vila-Rodriguez, Ochoa, Autonell, Usall, \& Haro, 2011).

\section{Recovery Styles}

Different strategies or coping responses for managing psychosis have been identified. Different recovery styles have been explored with some individuals integrating their illness experiences into their life stories and self-identity and others sealing over or compartmentalizing the experiences such that they are treated as a life disruption. Sealing over can be regarded as one way to reduce negative emotional states and maintain equilibrium during acute phases of illness (Perry, Taylor, \& Shaw, 2007). One study of 
first-episode psychosis patients investigated how recovery style influenced recovery outcomes. They were assessed at stabilization and again at 12 months. The most common recovery style for those with affective disorders was integrative while the most common style for individuals in the schizophrenia spectrum was sealing over. Those with sealing over styles had worse scores for Quality of Life and symptom rating scales. Females were more likely to have integrated styles than men. It was noted that some individuals changed their recovery styles over the study period which indicated an area for intervention (Thompson, McGorry, \& Harrigan, 2003). As those with sealing over recovery styles are known to engage in more denial related to their illnesses, they are thought to have worse prognoses. However, one study found that individuals with the highest levels of recovery were also more likely to engage in denial or avoidant coping. The researchers speculated that such a coping style may be adaptive under conditions that one cannot change such as stigma and discrimination (van Gestel-Timmermans, Brouwers, Bongers, van Assen, \& van Nieuwenhuizen, 2011).

\section{Social Disability}

Social disability is defined as disturbance caused by impairments in performance of specific roles that would normally be expected of individuals by their community. Social disability status was assessed for individuals with schizophrenia, schizoaffective, and mood disorders fifteen years after a first admission to a psychiatric hospital. Despite differences in severity of social disability, the profiles of the individuals in the different diagnostic groups were almost identical. That is, disability across diagnostic groups was of similar quality and pattern. What explained severity was the presence of negative 
symptoms or apathy which apparently led to social withdrawal (Bottlender, Straub, \& Moller, 2010). Another study of individuals who had been hospitalized due to episodes of mental illness had similar findings. Symptom severity and not type of diagnosis was again found to be the most significant factor for level of social disability. Social disability at both time of hospital admission and three months post discharge was lower for those who had professional careers and those who were in stable partnerships. The crosssectional nature of the study limits conclusions regarding direction of effects. Better functioning may have resulted in maintaining an occupation and engaging in a partnership rather than work and partnership resulting in better functioning (Rymaszewska et al., 2007).

It is widely recognized that individuals with serious mental health issues often lack opportunities for social interaction and meaningful activity. A study of social processes that support recovery found that individuals who perceived that their involvement in social activity meant something to others were more likely to continue with the social engagement. Having meaningful and routine activity was significant for ongoing social participation (Yilmaz, Josephsson, Danermark, \& Ivarsson, 2009). Borg and Davidson (2008) found that spending time in ordinary social settings and fulfilling common social roles such as in one's family and hobbies and vocations supported recovery as individuals took on challenges and developed coping skills. Daily occupations have been found to be sites for the formation and maintenance of social relationships. Individuals in recovery have reported that having occupational roles is important in positive self-regard and the development of social lives (Lencucha, Kinsella, \& Sumsion, 2008). A systematic review 
of the qualitative literature on work and recovery found that occupation of vocational roles supported recovery by providing a sense of living a normal life and the ability to meet social expectations, supporting the development of a worker identity as opposed to a patient identity, building a sense of social belonging, and through the earning of financial means with which to access social and cultural activities (Walsh \& Tickle, 2013). Supported employment programs that follow a social model of recovery view employment as part of the journey of recovery rather than as a goal that must await recovery (Secker, Membrey, Grove, \& Seebohm, 2010).

\section{Older Adults}

A study of older adults diagnosed with schizophrenia and schizoaffective disorder living in the community found that $49 \%$ met criteria for symptom remission. Findings converged with those of similar studies. Such remission rates are higher than those found in younger populations. In terms of community integration, the individuals were only doing half as well as their matched peers in the general community (Cohen, Pathak, Ramirez, \& Vahia, 2009). Older adults describe an illness course of early loss and life disruption, a period of adaptation and skill building, ongoing social isolation issues, and symptom improvement over time. Individuals attained greater capacity to manage psychosis over the lifespan but were often disappointed with the state of their relationships and with life achievements (Shepherd et al., 2012). An evaluation of the value of the recovery concept to older adults found that the process of ongoing recovery was connected to the extent to which a sense of identity could be maintained. Older adults in recovery perceived that they were managing well to the extent that they had a 
stable sense of self. The participants spoke of struggles with social and vocational roles, the impact of physical health issues, and the importance of constructing a coherent narrative of illness experiences (Daley, Newton, Slade, Murray, \& Banerjee, 2012).

\section{Social Supports}

Higher educational achievement, occupational or work roles, and stable relationships were associated with better social and functional levels in one large study $(N=926)$ of individuals diagnosed with schizophrenia in outpatient treatment. However, the crosssectional study design limits drawing conclusion regarding directionality of effects (Mohr et al., 2013). In an effort to explore how clinical (absence or control of symptoms) and non-clinical (positive self-identity, social engagement) dimensions of recovery impact participation in community activities, it was found that non-clinical recovery was significantly related to community activities. Further, when both psychiatric distress and non-clinical recovery were entered into a regression model predicting community activities, psychiatric distress became non-significant. The mediating model suggests that while psychiatric distress may be important initially, improved non-clinical recovery will account for more community activity. The researchers asserted that the findings support integration of symptom management and recovery-focused services (Davis, Townley, \& Kloos, 2013).

One study of individuals with schizophrenia-related diagnoses explored the relationship between (a) objective symptom severity and level of functioning, and (b) subjective personal recovery including social support and loneliness. No significant correlation was found between symptom scores and recovery scores or between 
functioning and recovery scores. Social support was significantly correlated with recovery and quality of life whereas loneliness was negatively correlated with recovery and quality of life. The researchers concluded that the study results were consistent with literature suggesting that symptomatic recovery is not synonymous with self-assessments of being in a state of recovery (Roe, Mashiach-Eizenberg, \& Lysaker, 2011).

A study of participation in a consumer-run organization focused on the relationship of perception of available social support and recovery. The shared experience of having a mental illness was the essential element involved in bonding and the development of supportive relationships. Everyone shared experiential expertise. The availability of volunteer activities and leadership opportunities on entry into such organizations allowed individuals to contribute immediately to the community as equals. The study found that both socially supportive and leadership participation experiences were related to progress with recovery (Brown, Shepherd, Merkle, Wituk, \& Meissen, 2008). Such settings have been found to be important for rebuilding a disrupted social network and forming relationships that are symmetrical in power (Schon, Denhov, \& Topor, 2009). Sense of Self

Individuals who have experienced significant mental distress have been found to have a diminished sense of a comprehensible life story and difficulty perceiving the self as socially connected and effective (Lysaker et al., 2006). One's sense of self is tied to one's life story and the meanings generated by it. Developing one's story involves making sense of life events. It is important to note that as we are social creatures, self narratives are best constructed not in isolation, but through dialogue in social relationships (Barker, 
2003; Lencucha, Kinsella, \& Sumsion, 2008). When symptoms are not viewed in social context, they come to be taken as reflective only of individual psychopathology. The next step can be equation of the individual with the illness (Borg \& Davidson, 2008). An illness-dominated identity can emerge from discrimination and stigmatized notions of mental illness that result in alienation and social exclusion. It has been found that illnessdominated identities have been resisted by reaching out for help with constructing alternative understandings of mental distress and regaining a sense of self as separate from the illness experience. Some have done so through involvement in consumer communities that resist medical conceptualizations of mental distress and that promote pride and self-determination (Mancini, 2007).

Social recovery appears to involve moving from a passive to an active sense of self. Aspects of an active sense of self include determination to recover, optimism or hope, taking responsibility for the self, and accepting and managing mental distress. Individuals in recovery have stated that illness was a transforming experience in which an old self was let go and a new more functional self was constructed. Acceptance by friends and family and social involvement supported the process (Topor, Kalyanasundaram, Glover, \& Momenzadah, 2003). Individuals who have meaningful accounts of their life experiences have been found to be more socially connected. It may be that life narratives form the bases for the ability to form social relationships (Lysaker, Ringer, Maxwell, McGuire, \& Lecomte, 2010). Individuals have described recovery as a social process that was best fueled by the communication of expectations that they could pursue goals and make a social contribution. They emphasized the importance of not being viewed through 
a disability lens and not being told to let go of plans for the future (Mancini, Hardiman, \& Lawson, 2005).

Individuals report that they need a life outside of a treatment identity. Social interactions where one is reduced to a disorder function as barriers to recovery. A positive sense of self is one that is no longer equated with the illness experience (Mancini, Hardiman, \& Lawson, 2005). Some have framed the equation of self with illness as "psychiatric colonization" (Barker, 2003). One analysis of recovery narratives found that reconstructing one's life involved externalizing the illness experience such that psychiatric issues affected, but no longer constituted, the self. Psychiatric problems became a fact of life but not the whole or core of one's life (Ridgway, 2001). Social relationships that support recovery allow individuals to redefine themselves as not just a patient but an individual with both needs and abilities. Helpful relationships are reported as involving consistent support, opportunities to make a contribution, and freedom for decision making. Familial and other enduring relationships can remind the individual of life before psychiatric disability and friendships with other individuals in recovery can allow for relationships that feature empathy and reciprocity in terms of both receiving and providing support (Schon, Denhov, \& Topor, 2009; Topor, Borg, Girolamo, \& Davidson, 2009). Individuals in recovery who have become mental health professionals have stressed the difficulty of overcoming stigma, hostile attitudes, and other societal barriers to recovery. They do not see that their recovery is primarily a function of how disabled they are, but perceive that how they have been and continue to be treated in society is a major barrier to recovery (Frese, Knight, \& Saks, 2009). 


\section{Meaning in Life}

Consumer definitions of recovery focus on processes of personal growth, selfdirection, and development of a meaningful life (Jaeger \& Hoff, 2012). That mental illness experiences are not wholly negative and can potentially enrich or add meaning to someone's life is a relatively new idea in the literature. One review of available research on meaning in life and recovery found that perceiving mental illness experiences as meaningful was correlated with hopefulness and a valued sense of self (Stickley \& Wright, 2011). Individuals report that they needed to explore the impact of the illness experience on themselves and their lives (Bradshaw, Armour, \& Roseborough, 2007). The process of recovery involves development of a purpose beyond psychiatric disability. Developing meaning from mental distress involves learning to interpret or reinterpret experience and find a way to lead a worthy life. Meaning-making helps make the distressing, disorganizing experience intelligible and places it in life context (Or et al., 2013; Schon, 2009; Ware, Hopper, Tugenberg, Dickey, \& Fisher, 2007).

The development of life narratives help individuals make meaning out of experience (Lysaker, Ringer, Maxwell, McGuire, \& Lecomte, 2010). The search for meaning has been found to be highly idiosyncratic (Perry, Taylor, \& Shaw, 2007). The act of telling one's narrative can facilitate a process in which one integrates disruptive, traumatizing, and stigmatizing experiences so that one's sense of self is broadened rather than limited by experience. Difficulty can be transformed into significant life experiences and the sense of self can include the psychiatric disability without being centered or defined by it (Onken, Craig, Ridgway, Ralph, \& Cook, 2007). The consumer perspective maintains 
that meaning develops through involvement in work, social relationships, and pursuits of goals. Participation in ordinary community arenas such as family and work settings has been found to provide a sense of meaning in life to individuals in recovery (Borg \& Davidson, 2008). Individuals have reported that they derive meaning by exploring their illness experiences and the consequences for their lives. The exploration is done by expressing their thoughts and the emotional content of their symptoms and related life events in treatment, in supportive relationships, and/or through expressive mediums (Schon, Denhov, \& Topor, 2009).

Schon (2009) interviewed men and women in recovery regarding how they found meaning and how the meaning-making influenced their recovery. Finding a cause for the illness was a core factor and could involve a combination of biological vulnerability, early losses and trauma, and relational stress. One study of 60 individuals diagnosed with serious mental illness explored the relationship between meaning in life, insight into mental illness, and internalized stigma. There was a significant negative correlation between internalized stigma and meaning in life. That is, people with higher levels of internalized stigma reported having a less meaningful life. Internalized stigma moderated the relationship between insight and meaning in life. Those who had moderate to high internalized stigma and high insight reported a less meaningful life. Those who had low internalized stigma and high insight appeared to be able to develop a sense that their lives had meaning. Internalized stigma appeared to hinder an individual from acquiring the sense of meaning in life. The researchers concluded that interventions designed to reduce 
self-stigma could support development of a sense of meaning in life and support the recovery process (Or et al., 2013).

As currently defined, insight means holding to a clinical illness model. A recoveryoriented understanding of insight would be inclusive of a wide range of beliefs and expectations of mental distress and healing journeys (Schrank, Bird, Rudnick, \& Slade, 2012). Individuals who conceptualize recovery narratives not solely as matters of personal agency, but also as social and political concerns, have been found to have strong recoveries. Such individuals have emphasized the importance of having meaningful relationships, a purpose in life, and being of service to causes greater than the self. Helping others was seen to give life meaning (Adame \& Knudson, 2007).

\section{Summary}

The outcome literature provides evidence that recovery concerns social involvement and a sense of connection. Individuals recover in social context and require meaningful activity and occupation of social roles. Recovery concerns an individual seeking affiliation and understanding, developing meaning from experiences, and constructing an effective self. Individuals need to lead purposeful lives in supportive social arenas. The next section explores social capital and social inclusion. Attention will be given to the community participation literature and the effects of involvement in peer-programming.

\section{Social Capital and Social Inclusion}

The term social capital is often used as an umbrella term to include social support, community participation, and social inclusion. It has been described as the "soft infrastructure that constitutes community capacity" (Dillard, Dujon, \& King, 2009, p.4). 
Social capital is characterized by community networks, civic identity and engagement, norms of cooperation, and trust in the community. It has also been defined as the resources and power that are embedded within social networks (Webber et al., 2013; Whitley \& McKenzie, 2004). There are two main types: bonding and bridging. Bonding concerns social networks that feature shared values, mutual trust, and reciprocity. Bridging social capital involves connections between different social groups, access to public goods and services, and the cognitive component of sense of belonging. Bonding social capital could be said to help people get by, while bridging social capital could be said to enable people to get ahead or continue to strive towards higher goals. Building social capital and increasing social inclusion have become goals of mental health policies in some countries, of the World Health Organization (WHO), and of the World Bank. An interdisciplinary review of primary studies on social capital and mental health noted that social capital is a multi-dimensional construct inclusive of social support, social cohesion, and other social determinants of health. There are complex associations between social capital and mental health. Mental well-being is related to issues of economic and social inequalities involving class, gender, and race. Almedom (2005) asserted that given the conceptual ambiguity of mental health, measurable associations between social capital and mental health can only be approximate. Others have warned against some of the apparent conflating of social capital with recovery (Duff, 2012) and advocate for treating social capital as an ecological issue as differentiated from an individual matter. Moreover, most studies have relied on aggregated data collected from 
individuals which may result in missing important dimensions of community life (Whitley \& McKenzie, 2005).

A systematic review of the literature on social capital and mental illness found strong evidence of a negative association between bonding social capital and mental illness. A higher level of bonding social capital involving social support and reciprocal relationships was associated with lower risk for mental illness. Reviewed studies were cross-sectional which limits conclusions regarding direction of effects (De Silva, McKenize, Harpham, \& Huttly, 2005). A more recent US study’s findings were convergent as bonding social capital was significantly associated with mental health. Participants found that it was more important to feel that one had access to social resources in the event of need than to have frequency of actual contact (MalmbergHeimonen, 2009). A national cross-sectional survey in Japan $(N=5,956)$ found that high levels of trust and high levels of community group membership were associated with better mental health after adjusting for age, sex, household income, and educational attainment (Hamano et al., 2010).

Social resources have been identified by individuals diagnosed with mental illness as the most important type of resource enabling recovery. Recovery takes place in the everyday world of social contacts. Participants in one study described the onset of mental illness as a period of significant social disconnection, and social contact as the most effective way of addressing the disruption. Social and family connections sustained recovery and generated hope for the future. Social connections also helped with access to needed material resources (Duff, 2012). 
There has been little focus on community development as a means of mental health promotion. Starting from an understanding that mental health problems in disadvantaged communities occur within a context of social and economic marginalization, a mental health promotion program was developed in order to focus on bringing local residents together for purposeful action. It was hoped that bringing people together would create an opportunity for exploring shared values and developing common understandings of conditions and issues. The workers proposed that their framework merited evaluation and further development although they did not directly measure mental health symptoms or functioning (Rose \& Thompson, 2012).

Social engagement appears to be a factor in the building of social capital. However, discrimination has been seen to restrict access of individuals with mental illness to social networks and capital. One large $(N=1016)$ study of mental health service users in England found that the majority reported experiencing discrimination and that they had lower access to social capital resources compared to the general population. The researchers stated that longitudinal studies were needed to establish directionality of effects (Webber et al., 2013). The most frequent experiences of stigma have been reported not from strangers but from family and friends. Individuals have reported that feeling misunderstood or defined by mental illness can lead to social retreat (Bromley et al., 2013). Some who have reviewed the literature on stigma and social exclusion of individuals with mental illness have concluded that if an individual has a social role that is understandable to others, he or she is more likely to be perceived as an acceptable member of society. It therefore becomes incumbent on society to support role occupation 
of those with mental health challenges (Baumann, 2007). There are currently no validated screening instruments for measurement of social capital. As mental illnesses often result from a culmination of injuries over a lifespan, longitudinal studies are particularly indicated (Whitley \& McKenzie, 2005).

\section{Social Inclusion/Exclusion}

The intersection of mental illness, social stigma discrimination, and poverty create significant barriers to social inclusion (Bradshaw, Armour, \& Roseborough, 2007). The World Psychiatric Association maintains that stigma and social exclusion are greater barriers to good quality of life for mental health consumers than the illnesses themselves (WPA, 2012). Similarly, Western governmental systems officially maintain that stigma and discrimination form the greatest barriers to social inclusion and recovery for individuals with mental health problems and histories (Barnes, 2011; New Freedom Commission on Mental Health, 2003). Social inclusion is a stated goal of mental health policy and services in the United States. It involves the engagement of individuals with psychiatric disabilities in social interactions in normative settings and equal access to opportunities offered to other members of society (Wong, Sands, \& Solomon, 2010). Social inclusion involves many life domains including community participation, social networks, access to education and employment, and stable housing. While there is no commonly agreed-on definition of the term, social inclusion has both objective and subjective elements. The objective element concerns the extent to which an individual participates in various life domains and may be measured by counting time spent involved in community activities and the number of available social contacts. The 
subjective element concerns the individual's preferences regarding community participation and social networks as well as his or her sense of inclusion (Coombs, Nicholas, \& Pirkis, 2013).

Social inclusion has been seen as relational and context-dependent. It involves material and social inequalities, relative deprivations, and subjective experiences. The concept has origins in French Republican thinking of the 1970s which framed the issue as one in which there had been a breach of social justice. Disconnection from mainstream society went beyond poverty and included non-participation in politics, poor health, and geographic isolation. Social exclusion is a broad understanding of deprivation that involves loss of roles and meaningful relationships and discrimination that can both precede and accompany mental illness. A widely cited definition of social inclusion for individuals with mental health challenges concerns improved rights of access to the social and economic world, new opportunities, recovery of status and life meaning, and reduced impact of disability. Becoming socially included involves being able to fulfill social roles centering on employment, voting, and social activities (Wright \& Stickley, 2012). The theoretical work on mental illness and social class features two main hypotheses: the drift hypothesis and the stress hypothesis. The drift hypothesis maintains that the onset of mental disorders leads to social disadvantage including reduction in social role occupation, and the stress hypothesis maintains that living in disadvantaged areas produces or amplifies mental distress. It may be that both hypotheses operate to varying degrees (Gould, 2006). 


\section{Citizenship}

Citizenship is a key concept within the notion of social inclusion and is defined as collective rights and responsibilities. Some have asserted that voting may be the real measure of how well individuals have integrated into society. The social role of voter may be a point from which to challenge stereotypical images of the deviant and dangerous mentally ill (Nash, 2002). A study of political engagement found that voting was seen as a powerful symbol of overcoming historical and contemporary discrimination. Helping one another to vote and engage in other political activities was viewed as building solidarity and community (Bergstresser, Brown, \& Colesante, 2013).

Social inclusion theorists regard elements of social inclusion as being both causes and consequences of mental illness (Nash, 2002). One conceptual and methodological review of social exclusion and mental health found arguments that much of the apparent social impairment of those with mental health challenges was a function of societal responses. Therefore, social inclusion could only be achieved through social change (Morgan, Burns, Fitzpatrick, Pinfold, \& Priebe, 2007).

While assessment of social inclusion outcomes is called for by different stakeholder groups, a lack of conceptual clarity poses a problem for measurement. At present, quality of life is the most frequently used concept in social outcomes in the psychiatric literature. Existing scales tend to capture mental illness symptoms and physical health indicators. Labeling these matters as quality of life has been questioned (Priebe, 2007). Social exclusion is associated with detachment of individuals and groups from the mainstream of society and is characterized by multiple deprivations such as restriction of rights, 
discrimination, and lack of material resources. It involves a multidimensional and relational process that encompasses both economic and non-economic domains of wellbeing. Given the complexity of the construct, social exclusion may warrant multiple indicators as opposed to a single index or scale (Zelenev, 2011).

A systematic review of the peer-reviewed literature on social inclusion and mental health found that social inclusion involved capacity to fulfill social roles such as employment, voting, and social activity (Wright \& Stickley, 2012). A social work literature review (Farone, 2006) emphasized that the population of individuals with mental health diagnoses was at high risk for high stress levels and for low levels of access to social coping resources. The social inclusion argument was explored as concerning exclusionary processes resulting from the social construction of position, roles, and statuses. Her review of empirical studies found that individuals required skills to cope with stigma and symptoms, and benefited greatly from developing an active sense of self that was separate from debilitating symptoms. Individuals stressed the need to selfdirect, engage in normal activities, and develop meaningful social relationships.

\section{Social Sustainability}

Social sustainability concerns policies and institutions that effect integration of diverse groups and cultural practices in a just and equitable fashion. Principles include human well-being, equity, and civil involvement (Dillard, Dujon, \& King, 2009). The social sustainability concept is used to describe a collective understanding of the need to develop and maintain communities that allow individuals to thrive through equal access to opportunities for development. The principles of social sustainability include equity of 
access to resources and key services, equity between generations, diversity, interconnectedness, democracy and shared governance, and quality of life. Valuing diversity as a principle of social sustainability refers to a community's preparedness to seek diversity and value difference rather than homogeneity. Placing value on diversity leads to challenging assumptions about population groups and can build the social imagination about what is possible (Hammond \& Churchman, 2007).

Disability issues are rarely addressed in the academic social sustainability literature. What does exist focuses on equity to access to key services such as transportation and recreation (Wolbring \& Rybchinski, 2013). However, in social sustainability, mental health is viewed as a social indicator and as foundational to social well-being. As such, its cultivation requires community as well as individual level interventions. Lack of equity is seen as a key barrier to mental health. Mental health develops in social environments that are shaped by distributions of power and resources. Positive mental health is supported by positive social institutions that increase social connection through access to material needs, valued social roles, and political voice (Lindahl, Balajee, \& Wiggins, 2013).

Sustainability in mental health is being conceived of as involving recovery capital. The conceptual framework of recovery capital concerns those elements that are important in enabling an individual to attain and reclaim both personal efficacy and social capability. Internal and external resources can interlock to make progress with recovery. The resources that enable recovery include supportive relationships that involve some degree of reciprocity such that the recovering individual can make a contribution and be 
valued, social networks, economic means, a positive self-concept, and coping skills (Tew, 2012). Related to the value social sustainability frameworks place on diversity, there is a developing discourse on madness that concerns mental diversity (Wolframe, 2013). Celebration of mad culture involves the reclamation of terms such a "mad" and "lunacy" and regards madness as a culturally meaningful and active minority identity. The experiences of people who have experienced madness are regarded as having the potential to inform the broader society (Schrader, Jones, \& Shattell, 2013).

\section{Positive Psychology and Recovery}

Positive psychology has been defined as the science of what is needed for a good life (Slade, 2010). Similar to the recovery movement's shift of focus from symptom reduction to attainment of a life of meaning and purpose, the focus of positive psychology is on personal fulfillment and well-being, not illness or pathology (Moran \& Nemec, 2013). The goals of the recovery movement that concern people re-engaging in life on the basis of their own goals and strengths and reclaiming valued identity and valued social roles appear congruent with the goals of positive psychology (Schrank, Browne, Tylee, \& Salde, 2014). The discipline studies sources of human strength and the elements needed for leading a high-quality life (Resnick \& Rosenheck, 2006). The founder of positive psychology, Martin Seligman, asserted that existing theories were powerful for predicting failure and despair but inadequate for explaining hope, compassion, and other qualities that make life worth living. He drew his theory from studies of the protective effect of social support on psychological disorders and distress (Gillham \& Seligman, 1999). In addition to attention to subjective experiences of the individual with contentment, hope, 
and capacity, positive psychology examines societal institutions that support active citizenship and involvement in vocational pursuits (Seligman \& Csikszentmihalyi, 2000). Positive psychologists have asserted that while difficulties should not be denied, sole attention to disorder leads to an incomplete view of the human condition. Problems may co-exist with assets and strengths. The perspective maintains that even if problems cannot be resolved, there are many routes to a good life (Peterson, 2009). In this respect, positive psychology is aligned with the social model of disability which views disability as a growth experience and the developing madness theory base which includes a focus on posttraumatic growth by those who experience psychosis (Dunkley \& Bates, 2015; Livneh \& Martz, 2015).

In order to infuse positive psychology into current models of mental health treatment, it would be necessary to include strengths and positive traits in the study of mental health issues, assess the extent of positive factors in the environment, resources, and opportunities, and measure subjective well-being and increases in positive coping in addition to reduction of symptoms and negative behavior (Lampropoulos, 2001; Slade, 2010). Research could benefit from using positive psychology indicators to examine recovery factors that may be independent of symptom reduction (Moran \& Nemac, 2013). Positive mental health and mental illness may be complementary dimensions, not merely opposites. A longitudinal panel study with a large adult sample $(N=1932)$ measured both psychopathological symptoms and positive mental health at four points over nine months found that changes in psychopathology were predictive for levels of positive mental health and vice versa. The presence of psychopathology appeared to be a 
risk factor for low well-being and those with low well-being appeared to be at risk to develop psychopathological symptoms (Lamers, Westerhof, Glas, \& Bohlmeijer, 2015). A study that compared levels of happiness reported by individuals diagnosed with schizophrenia with those reported by healthy controls found that while the schizophrenia group reported lower happiness, the overall pattern of happiness correlations was very similar in the two groups. Happiness was associated with lower perceived stress, higher resilience and optimism, and higher personal mastery, but not with age, education, physical health, cognitive functioning, or, among the schizophrenia group, duration of illness or positive or negative symptoms. Additionally, 37\% of individuals with schizophrenia scored in the high range on the happiness measure used (Palmer, Martin, Depp, Glorioso, \& Jeste, 2014). However, individuals who experience persecutory delusions have been found to report well-being scores that are significantly lower than those of health controls (Freeman et al., 2014). Such findings support continued investigation of the role of positive emotions and recovery from mental health challenges.

A number of techniques have been developed to encourage people to identify and further develop positive emotions, experiences, and character traits. A meta-analysis of 39 studies found that positive psychology interventions significantly enhanced subjective and psychological well-being and reduced depressive symptoms (Bolier et al., 2013). A positive psychotherapy group for individuals with schizophrenia was found to result in increased hope and well-being and decreased paranoid, psychotic, and depressive symptoms at post-treatment and at three-month follow-up (Meyer, Johnson, Parks, Iwanski, \& Penn, 2012). A qualitative study of another 11-week group positive 
psychology intervention for psychosis found that participants reported that it was helpful to focus on positive things rather than ruminating on the negative and that identifying and developing strengths helped with overcoming obstacles and made them feel more able to cope with symptoms (Brownell, Schrank, Jakaite, Larkin, \& Slade, 2014). A loving kindness meditation designed to increase feelings of warmth and caring for self and others delivered to individuals with schizophrenia was found to lead to large improvements in frequency and intensity of positive emotions and large decreases in negative symptoms at post-treatment and three-month follow-up (Johnson et al., 2011).

\section{Peer Programs}

Community involvement for individuals who are in early recovery from mental illness involves a number of experiences. Communities are defined as places where they can receive help, have the opportunity to identify with others in a social group, and secure shelter from stigma and discrimination. Individuals in recovery have been known to describe individuals in their support community as members of their family. Involvement with peers in community provided opportunities to both give and receive support and was a means of valuing illness experiences by using them to help others. The reciprocity of the relationships was highly valued in and of itself. Peer relationships appeared to facilitate involvement in the mainstream community (Bromley et al., 2013; Wong, Sands, \& Solomon, 2010).

Peer support programs involve the employment of individuals with lived experience for provision of experience-based services. A central intention of the peer support role is to model recovery and thereby communicate hope that recovery is possible. Employment 
of individuals in recovery communicates that the experience-based knowledge possessed by the service user is valuable (Smith-Merry, Freeman, \& Sturdy, 2011). Individuals are recognized as more than the illness and there is no pressure to become symptom-free before participating in the peer community (Farone, 2006). Peers provide individuals with both acceptance and advice about how to reach recovery goals and offer a road map for recovery journeys. In contrast to treatment relationships in which they only receive support, peer support programs and communities feature relationships in which they both give and receive. Consumers identify this aspect of relationship as key to the benefit they receive from involvement in peer programs. Being able to help others promotes a sense of purpose and well-being (Borg \& Davidson, 2008; Lencucha, Kinsella, \& Sumsion, 2008). Individuals gain a sense that they can be something more than a patient, can engage in activities they did not think were possible, and can access opportunities to develop further as a person (Bradshaw, Armour, \& Roseborough, 2007; Mancini, Hardiman, \& Lawson, 2005). Making use of their experiences through helping others has been described as a key turning point in recovery by some individuals involved in peer programs and as a means of sustaining one's recovery (Mancini, 2007; Onken, Craig, Ridgway, Ralph, \& Cook, 2007).

The elements of peer-run programming that have been found to be effective include sharing of experiential knowledge, availability of role models of recovery and healing, and social support and valued organizational roles. Such programs have been found to increase self-perceptions of empowerment (Rogers et al., 2007). Conceptually, empowerment is a process by which individuals who have lesser power in a society gain 
control over their lives and the ability to influence their lives (Swarbrick, 2009).

Empowerment is a relatively new recovery concept in the literature (Resnick, Rosenheck, \& Lehman, 2004) and is the conceptual opposite of self-stigma (Rusch et al., 2014). Some have described stigma as the "social embodiment of disempowerment" (Bradshaw, Armour, \& Roseborough, 2007, p.28) while others have described recovery as a manifestation of empowerment (Onken, Craig, Ridgway, Ralph, \& Cook, 2007). Despite its recent arrival in the peer-reviewed literature, empowerment has been identified as a key theme in subjective accounts of recovery by consumers (Pitt, Kilbride, Nothard, Welford, \& Morrison, 2007). Elements of empowerment include decision-making power, access to information and resources, hope, emotional regulation and communication skills, the unlearning of social conditioning regarding chronic illness, self-efficacy, and a positive self-concept (Cohen, 2005). Peer support programs are known for broadening the concept of recovery from a personal endeavor to a relational and political one (Adame \& Knudson, 2007).

\section{Community Participation}

Individuals diagnosed with psychotic illnesses are known to experience greater social exclusion than individuals with other mental illnesses. A survey of individuals diagnosed with schizophrenia or schizoaffective disorder found that in addition to statistically significant reductions in social integration and productivity after the onset of illness, barriers to involvement cited by participants more often concerned self-confidence and self-esteem than direct discrimination or pragmatic access issues. The researchers concluded that findings indicated the need for interventions to address self-stigma 
(Killaspy et al., 2013). Young adults with psychiatric disabilities may lead confined lives and have few opportunities to meet friends and participate in communities. This may complicate the process of individual development towards adulthood. One study explored where young people with psychiatric disabilities spent their time and what relationships were associated with different places. Having access to many places in different social arenas provide opportunities to establish a range of social relationships. The range of social relationships allowed for individuals to exercise independence and autonomy, become part of communities, and develop a sense of belonging. Involvement in social relationships promoted adult development (Olin, Nordstrom, \& Hijk, 2011). Both emerging and mature adults with mental illness have been found to have stronger meaning of life, quality of life, and recovery outcomes with greater community participation. The impact of participation is the same for both groups. Such study results support the development of policy that directly promotes social inclusion (Kaplan, Salzer, \& Brusilovskiy, 2012).

The concept of social inclusion has been criticized as being so focused on where individuals are physically located that it fails to capture the social dimensions of a sense of connection. Social inclusion is becoming defined as not only concerning access to housing, education, and employment but also as involving a sense of belonging and a sense of self beyond disability. What it means to be socially included is subjective, relative, and fluid as opposed to a fixed state of being (Le Boutillier \& Croucher, 2010). Those who are members of community groups besides mental health treatment and support communities report improved self-concept and greater sense of social inclusion 
(Wong, Sands, \& Solomon, 2010). Such community involvement helps individuals feel that they are leading normal lives while contending with disability issues (Bradshaw, Armour, \& Roseborough, 2007).

One qualitative study of individuals who had recovered from psychiatric disability aimed to develop a new definition of social integration. Social integration was defined as a process that unfolded over time through which those who had been psychiatrically disabled developed and exercised capacities for connectedness and citizenship. Connectedness involved reciprocal interpersonal relationships and citizenship referred to the rights and responsibilities shared by members of a democratic society. Connectedness and citizenship involved not only emotional aspects but access to social and material resources as well. Implied in the definition is the possibility for growth and development rather than just stabilization in a community (Ware, Hopper, Tugenberg, Dickey, \& Fisher, 2007). Individuals in recovery have reported that they experience contingencies on their citizenship. When others are aware of their diagnoses, they are often excluded due to being viewed as lacking competency and/or intelligence. They report that others appear to regard them as feared outsiders. The ongoing rejection and exclusion are reported as taking a toll on mental health and leaving individuals feeling exhausted. In order to be included as citizens, many hide their diagnoses even though doing so can result in emotional conflict and feelings of self-betrayal (Hamer, Finlayson, \& Warren, 2013).

By synthesizing themes that emerged from the research on recovery, Mezzina, Davidson, Borg, Marin, Topor, and Sells (2006) posited a framework or map of recovery 
as an ongoing social process. Components of recovery included personal, interpersonal, and social domains, a sense of belonging, and material resources. The personal domain involved regaining a sense of control and exercising self-direction. Creating new habits and routines that allowed an individual to engage in normal activity was also part of this domain. Barriers included symptoms that were difficult to manage and both internal and external stigma. Recovery involved making sense of past experiences, redefining the self as having an identity beyond that of a mental patient, and exercising one's rights as a citizen. Essential elements included hope, a sense of purpose, and social interactions that enabled an individual to recognize capacities and strengths. Relationships could facilitate recovery through qualities of presence, consistency, and support. Community areas could support recovery through providing welcoming environments in which individuals could engage in activity they found personally relevant and meaningful. Social participation created a sense of commonality and belonging and enabled recovery by allowing individuals the opportunity to have a social identity. Material resources allowed access to meaningful activities that could help individuals break away from the illness experience. Work and volunteer opportunities provided material resources and a sense of accomplishment. The reconstruction of a sense of self took place in social contexts. They concluded that services needed to pay more attention to issues of social inclusion and civil rights and asserted that recovery is not a precondition of citizenship, but citizenship is a precondition for recovery.

People and their well-being cannot be separated from the social environment. One review of the literature on the impact of place on recovery emphasized that identity is 
associated with group membership formed within a place and around activity. Places regarded as "normal" are associated with positive identity and recovery. Place, activity, and identity elements can combine to support a sense of self distinct from an illness identity (Yates, Holmes, \& Priest, 2011). Social work concerns the individual in the environment and its tradition of anti-discriminatory practice is consistent with a focus on social inclusion. Such a focus can be seen as an opportunity to assert the relevancy of mental health social work and pursue a social justice agenda (Gould, 2006). Social inclusion is both an individual experience and a political issue (Topor, Borg, Girolamo, \& Davidson, 2009).

\section{Summary}

The literature reviewed in this section demonstrated that recovery is not just an individual phenomenon. Social resources and barriers to social inclusion such as discrimination act to either enable individuals or deprive them of their rights and responsibilities. The emerging concept of recovery capital places emphasis on the elements needed to attain personal efficacy and social capability. Peer-delivered services have been found to be one means of developing recovery capital. Involvement in peer services builds relationships that feature trust, authenticity, and reciprocity. Lived experience is valued as meaningful and individuals gain a sense of empowerment such that they can pursue their goals. The next section will explore the importance of consumer-involvement in research. 
Involvement of Individuals with Lived Experience in Research

The mad theoretical perspective and the recovery paradigm call for the involvement of individuals with lived experience in advocacy, services, and research. Development of all areas of the mental health field must be directed by individuals who have firsthand experiences of the phenomena in question. An understanding of mental distress and recovery will only be possible through involvement of those with lived knowledge. This section reviews literature on consumer involvement in research including the views of individuals with lived experience of recovery. Benefits, barriers, and risks of consumer involvement are reviewed. Issues addressed include the complexity of experience, standpoint, identity, the effect of power differentials, and the importance of relationship building and reflexive practice. Consistent with the disability and mad theory base, the consumer writers locate difficulties in the context of social oppression.

Research approaches differ in terms of greater or lesser distance and/or power shared between researchers and the participants. Emancipatory research involves changing and equalizing relationships between the researcher and participants and developing knowledge collectively (Telford \& Faulkner, 2004). The emancipatory research paradigm developed primarily in the disability field. However, emancipatory research and user-controlled research differ. While emancipatory research emphasizes equalization of research relationships, the focus of user-controlled research is on consumer ownership of all aspects of research including how it originates, who makes the decisions and conducts the research, how findings are disseminated, and what actions are taken based on findings. (Phillips, 2006). Some have termed research on mental health 
issues that is not determined by consumers themselves as one form of colonization of the consumer experience (Barker, 2003).

Beresford (2013) is a social work researcher with lived experience of recovery. $\mathrm{He}$ writes that what distinguishes the research approach of individuals with lived experience from more traditional approaches is the value placed on experiential knowledge. Experiential knowledge has long been devalued in the dominant quantitative paradigm which maintains that subjectivity compromises the credibility of findings. Involvement of individuals with lived experience of issues becomes framed as a threat to the legitimacy of research findings. Exclusion of such individuals from research is an "othering" process that results in further marginalization. Psychiatric disability approaches view research as inherently political rather than as neutral, objective activity. Research becomes focused on the improvement of the welfare of individuals rather than on simple generation of knowledge. Investigation of questions consumers themselves have and building their own knowledge are the primary concerns of consumer-directed research. These concerns are valued as consumers can inform themselves and build power based on knowledge. The central purpose of research becomes supporting the empowerment of the consumer and effecting social change.

Consumer involvement in mental health research has been framed as both a right and as a means to increase the relevancy and integrity of research (Happell \& Roper, 2007). The central argument for consumers' involvement in research is that their absence can lead to continued oppression through a reinterpretation of their lived experience (Phillis, 2006). Personal stories continue to be viewed as a powerful means to develop and 
validate key ideas and practices. The emerging literature emphasizes the importance of approaching people as individuals with unique journeys that hold much meaning (Ramon, Healy, \& Renouf, 2007). Involving consumers in research has become more of a practice standard and is included in institutional and governmental policies. Policy in the United Kingdom requires consumer involvement for much of public research funding and for governance bodies (Patterson, Trite, \& Weaver, 2014). However, a systematic review of both the peer reviewed and grey literature on consumer engagement in research concluded that the extent to which existing approaches actually ensured inclusion and whether there were consistent benefits to doing so remained unclear due to a lack of standard framework or language (Shippee et al., 2013). However, there is evidence that research conducted with consumer involvement is significantly associated with successful participant recruitment. The specific mechanisms have not been identified (Ennis \& Wykes, 2013).

Consumer involvement in research ranges from participation at the advisory level, to consultation and collaboration, and finally to consumer-led projects in which research is initiated and fully directed by individuals with lived experience (Happell \& Roper, 2007). Arguments for the inclusion of consumers in research include an ethical or moral argument, efficacy or quality enhancement, and assertions that participation in research has a positive impact on those involved. Involvement of consumers in pursuits that directly concern them is an ethical or moral issue. Inclusion is a matter of social justice. Further, involvement of consumers on research teams is argued to improve research quality by enhancing relevancy, methodological sensitivity, data collection, and validity 
of the findings. Consumers have reported positive feelings, skill development, and increased hope from participation in research (Hancock, Bundy, Tansett, \& McMahon, 2012). Barriers to consumer inclusion include the pace of the work, funding and manuscript deadlines, organizational policy, lack of training for researchers, and lack of ability to be responsive to consumer mental health needs. Therefore, even highly motivated researchers may face significant structural and knowledge barriers to collaboration with consumers (Staley, Kabir, \& Szmukler, 2012). Consumer-led or controlled mental health research remains rare in the peer-reviewed literature. More such research can be found in the alternative or grey literature (Telford \& Faulkner, 2004).

Some critical stances on consumer involvement in research acknowledge that while the manifest intention of participatory research approaches is to investigate reality in order to transform it, there is the potential that such approaches will be used in the maintenance of the status quo. Carey (2011) reviews how policy that requires consumer involvement in research does not address power differentials inherent in research roles and can lead to tokenism. Consumers involved in research may not be representative of other subgroups. Those who do become involved may be from groups that are valued more highly in society. Research itself holds limited power in effecting structural change. Certain research approaches may raise consciousness while not offering solutions. In the end, disempowered individuals may become more active in their subordination.

One exploration of how consumers involved as co-researchers experienced and valued their participation had a number of findings. The co-researchers held that one of their important functions was to examine the relevance and practicality of research topics and 
projects. Another responsibility they assumed was to widely communicate findings to people outside the research field who might benefit from the knowledge. They felt they could raise the quality of research by slowing the pace in order to take time to explore the potential implications of the direction research was taking. They viewed their task as one of building bridges between researcher and participant in order to improve communication. Researchers were asked to clarify their thought processes and intentions. The co-researchers reported that they felt empowered through engagement in the main project due to sharing their lived experience of distress and recovery (Moltu, Stefansen, Svisdahl, \& Veseth, 2013).

Researchers who identify as consumers can facilitate the involvement of lay consumers in the research process. They can highly value a range of consumer perspectives in framing relevant research questions and in designing acceptable research designs and protocols. Academics who are willing to openly identify as consumers can contribute to the reduction of stigma. However, consumer-identified researchers may offer primarily a mainstream or middle-class perspective and their stances on issues may be overly influenced by the dominant academic culture. Further, consumer researchers may be devalued by non-consumer researchers due to their experiences with mental distress while at the same time being viewed with suspicion and as no longer true consumers by individuals with lived experience who are outside academia (Griffiths, Jorm, \& Christensen, 2003). A survey of individuals with lived experience employed as researchers in the United Kingdom found that the majority believed that consumer involvement in research was necessary for building evidence for service intervention, 
improving the relevancy of mental health research, and empowering consumers. Survey participants expressed that involvement in mental health research had provided a sense of purpose and belonging, gave meaning to their illness-related experiences, and enhanced their self-respect. However, they also reported challenges due to being involved in a research field that included the devaluing of their lived experiences and related expertise. Further, they reported discrimination and exclusion from supports provided by consumers due to beliefs that someone could not be a member of both the academic and consumer communities (Patterson, Trite, \& Weaver, 2014). Some academics have critiqued consumer involved research as lacking objectivity and rigor. The ability of consumers to conduct research has been challenged based simply on their psychiatric diagnoses (Happell \& Roper, 2007). Some maintain that there is an inherent contradiction between being a researcher and having a mental health diagnosis as the former implies rationality while the latter implies irrationality (Telford \& Faulkner, 2004).

Researchers can never be fully removed from an investigation process that involves complexities of identity, social standing, and power. Knowledge is co-constructed. What becomes important in knowledge created among groups of individuals is what is shared. The process of bringing contrasting perspectives together leads to an emphasis on how parties relate to one another. Developing new ways of thinking about something allows for conversations about commonalities as well as differences. Such conversations can help disrupt the binary of us/them or the mentally healthy and the insane (Frankham \& Tracy, 2012). One review of the academic and grey literature found little exploration of non-consumer researchers' experiences of working on projects with consumer 
researchers. Further, the review noted that the fact that individuals involved in research may occupy more than one role has received minimal recognition. As power differentials are situated between group identities, those who claim more than one role or identity may challenge the distribution of power and thereby produce resistance in the academic system. Due to the challenging experience of holding multiple roles, some may understandably choose to keep personal and professional identities separate (Kara, 2013). There exists almost no empirical research that has assessed the level of challenges faced by researchers with psychiatric disabilities (Jones \& Brown, 2013).

An exploration of consumer involvement as advisors in a study found that it was important to take time in the course of research to build trusting relationships and commitment to the project. Taking time to explore the work and its potential impact also led to mutual learning for the consumers and the researchers. Consumer contributions increased the accessibility of research materials and enhanced the dissemination of findings. Recommendations included involving consumers in all stages of research. The study supported exploration of the subjectivity of all parties and the importance of reflecting on experience (Barber, Beresford, Boote, Cooper, \& Faulkner, 2011). Reflexive Practice

Reflexivity has been described as involving complex relationships between how we know, what we know, and who we are. It requires that investigators have multiple levels of awareness including of those factors that influence our internal and external reactions as well as our relationships. Such awareness allows us to appreciate how we co-create meaning. Reflexive knowledge involves not only knowledge about issues at hand but 
awareness of how the knowledge was acquired (Etherington, 2004). Reflexive practice also requires self-awareness. To be in relationship with another necessitates that one be in relationship with one's self. Being in relationship with one's self in turn involves the ability to be aware and tolerate thoughts and emotions as well as the ability to share one's genuine self with others. Relational work requires openness about one's vulnerabilities and limitations (Wang, 2012).

Reflexive practice can be used to recognize one's subjectivity as attached to such identities as gender, race, and sexuality. Such practice helps one to recognize and respond to differences in amount and kind of social power. Power relations can shift as individuals move in and out of various subjectivities. The relation can widen or narrow depending on the amount and kind of power operating at any one time and place. Instead of trying to eliminate subjectivity, reflexive practice can help with valuing and exploring subjectivity for how it shapes the interpretive and knowledge-building processes. Researchers who draw on transformative approaches such as feminism and disability theory recommend recording thoughts, ideas, and emotions that arise during the work. However, it is important to keep in mind that engaging in reflexive practice does not directly change power dynamics (Daley, 2010).

The consumer movement and consumer researchers advocate valuing the consumer voice as authoritative and building a knowledge base from people's subjective experiences. However, consumer researcher Adame (2012) has explored how her experiences and views made it difficult for her to hear the narrative of an individual who had had similar experiences but derived different meaning and dissimilar personal and 
professional identities. Her own thought processes and identity interfered with her understanding of how another consumer defined herself differently in relation to similar experiences. She concludes that it is important to learn how to tolerate ambiguity and complexity when exploring complicated issues.

\section{Complexity of Experience and Identity}

The heterogeneity of psychiatric disability experiences and intersecting ethnic/racial, religious, sexual, gender, class, and physical disability identities complicate consumer involvement in research. Impairments and symptom experiences carry differential interpersonal consequences. Further, the participation of persons of color in U.S. consumer research is quite low (Jones \& Kelly, 2014). Grounded in the social model of disability theory, Brown and Boardman (2011) who live with disabilities explored how the disability category involves a diversity of other identities and subjectivities. Multiple subjectivities are possible during interactions and identities can shift and be ambiguous. The personal identities of researchers influence power dynamics and can have profound impacts on the research process. Disability can be a marker of shared identity and can help bridge the distance between researcher and participant. Further, not only participants but researchers with devalued identities can experience vulnerability and even exploitation during the research process. Relationships of power can be played out through identity categories that contain relative social power. Power serves to privilege some positions while marginalizing others which makes for complicated relations between disabled researcher and research participant. It can be difficult to determine what identities will be prioritized in a research context. 
There is little in the literature on participant views of consumer-led research. One study explored how consumers experienced being interviewed by other consumers trained in conducting research. The study framed consumer involvement in mental health research as an emancipatory approach and as one avenue for amplifying the voice of a socially vulnerable group. Participants reported that being interviewed by another consumer involved a sense of equality or mutuality as both parties had had similar experiences with distress and social exclusion. There was a sense of comradeship and a perception that engagement in such research was supportive of the recovery of individuals in the roles of both participant and researcher. However, some participants reported that they did not trust other consumers based on past negative experiences with individuals who were highly distressed and had behaved in odd ways. Some reported feeling overwhelmed or dominated by the researcher's feelings, opinions, and narratives. Others did not want to share their experiences for fear of causing some sort of harm to the researcher. The authors concluded that findings pointed to an imbalance of power in some of the dyads and the existence of potential threats to validity of consumer-generated research (Bengtsson-Tops \& Svensson, 2010). The intriguing findings call for more investigation of this understudied area and the strengths and weaknesses of this approach to research.

\section{Summary}

Consumer involvement in research is a developing practice and is mandated in some policies and organizations. There exists theoretical support for the practice as well as significant practitioner and structural barriers to implementation. Further, empirical 
support is limited. Consumer involvement in research highlights both the sensitive nature of interpretation of experience and the need to manage power issues. The matter is further complicated by the diversity of experiences, subjectivities, and identities within the population of individuals with psychiatric disabilities.

Sharing experiences and providing testimony have been central to the history of organizing for resistance and change by individuals with lived experience of psychiatric disability. The consumer movement has long valued the potential of our narratives to challenge power if used collectively as political knowledge (Costa et al., 2012). In keeping with the tradition, consumer- directed research places value on experiential knowledge and certainly concerns generation of knowledge. The emphasis is placed on using knowledge as a basis for building voice and power. Research can be viewed as a political act. Claiming ownership of knowledge and discourse is a means by which to challenge the overarching narrative of the individual with lived experience of mental health challenges as lacking the ability to control his or her life and to exercise full citizenship. The value of consumer participation in research can be seen as the potential empowerment of a marginalized population through determination of knowledge and how that knowledge frames identity (Phillips, 2006). Recovery research and knowledge building provide a challenge to the traditional boundary set between the rational and irrational and the individual with lived experience of madness as a reasoning actor. The involvement of individuals with lived experience in research is an inherently radical effort (Topor, Borg, Girolamo, \& Davidson, 2009). The degree to which individuals with lived experience appear to have been involved in the development of recovery measures 
will be noted in the next section that reviews a selection of representative measurement instruments currently in wide use.

\section{Review of Measurement Instruments}

There is no gold standard by which to evaluate definitions of recovery and there is no consensus on which measures should be used to assess the different parameters of recovery (Jaeger \& Hoff, 2012). However, from the beginning of the consumer/survivor/ex-patient movement, individuals with lived experience of recovery have asserted that their views should guide the development of instruments used to evaluate interventions and measure outcomes (Tomes, 2006). In accordance, social dimensions in addition to psychological, symptomatic, and functional ones must be considered. A definition of recovery must include occupation of social roles and community engagement. A limitation of the literature on the consumer model of recovery is the lack of clarity regarding the extent that recovery is mediated or moderated by social outcome domains. The development of psychometrically-sound measures is needed to further the empirical study of consumer-defined recovery (Bellack, 2006).

Consumer descriptions of recovery generally include a focus on both process and outcome with outcomes inclusive of occupation of social roles and community engagement (Gordon, 2013). No social recovery instrument was found. However, instruments used with adults to measure recovery, functioning, and social inclusion will be reviewed given the relevancy to the instrument under development. Fifteen instruments in wide use were selected as representative of available measures. They were developed by respected and influential scholars. One of the main criticisms of traditional 
measures issued from consumers is that many have been developed by researchers and/or clinicians without genuine consumer input (Gordon, Ellis, Siegert, \& Walkey, 2013). The examination criteria utilized by Law, Morrison, Byrne, and Hodson (2012) will be followed as the criteria were developed in partnership with individuals with lived experience of recovery. The criteria are as follows:

1. factors/dimensions

2. psychometric robustness

3. level of consumer/mad input during development of the measure

4. inclusion of items relevant to consumer/mad concepts of recovery

5. positive framing of questions so as to speak to the presence of supports and capacities

6. user friendliness in terms of self-administration and ease of completion including positively worded items that lend clarity and ease of use, length of time to complete the measure, and ease of scoring.

The Stages of Recovery Instrument (STORI; Andresen, Caputi, \& Oades, 2006) was developed in Australia and is based on a definition of recovery as healing from the psychological trauma of illness rather than as a cure or the absence of symptoms. The developers looked to a stage of recovery model that includes loss and social withdrawal, developing awareness of the possibility of recovery, preparation for recovery, working towards a positive identity and goals, and growth or leading a full and meaningful life. The measure was created in order to empirically validate the model. Items were generated from the concepts represented by previous studies on the stages of recovery. The draft 
version of the STORI was piloted with six male and four female mental health consumerresearchers. Items were re-worded based on results of the pilot. The measure consists of 50 items presented in 10 groups of five. Response options range from $0=$ "not at all true now" to $5=$ "completely true now." The full investigation included a sample size of 94 individuals. Positive correlations were found between the STORI and the Recovery Assessment Scale (RAS; Giffort, Schmook, Vollendorf, \& Gervain, 1997) and the Mental Health Recovery Measure (MHRM; Armstrong, Cohen, Hellemann, Reist, \& Young, 2014) with a range of $r=.52$ to $r=.62$ and individual stage subscales were found to be internally consistent. The Cronbach's alpha coefficient test of internal consistency of the measure was .88. A cluster analysis of the STORI items found only a three-stage model instead of the expected five. The developers discuss the issue and assert that as the fivestage model has a sound basis in qualitative research, next efforts should be directed towards enhancement of the power of the instrument to discriminate between recovery stages.

The researchers did not claim to have lived experience and did not claim to have consumer advisors or consultants. Level of input for the development of the STORI appeared to be low as it was based largely on a review of the literature. However, the content of the scale appears to tap into aspects of recovery that are relevant to those with lived experience. That is, items address such issues as identity, personal growth, relationships, and self-determination of life purpose and goals. The STORI is a complicated instrument that requires close attention and some time to complete given the grouping of items into 10 different blocks. Items representing each stage are distributed 
though the 10 blocks which disrupts the flow of the instrument and complicates scoring. For example, block one includes the following items: I don't think people with a mental illness can get better; I've only recently found that people with a mental illness can get better; I am starting to learn how I can help myself get better; I am working hard at staying well, and it will be worth it in the long run; I have a sense of "inner peace" about life with the illness now. Response options are consistent throughout the instrument which does assist with completion. Many items are negatively framed. For example, one item reads, "I don't think people with mental illness can get better," and another reads, "I feel as though I don't know who I am anymore." Main criticisms of this measure concern its development to validate a stage of recovery model, the apparently low level of consumer involvement, the negative frames of items, and that it is a complicated instrument given how items are grouped into different blocks.

The Psychosis Recovery Inventory (PRI; Chen, Tam, Wong, Law, \& Chiu, 2005) is another measure developed in Australia. It is based on a model of recovery as involving ongoing processes, and concerns the early period of recovering from experiences with psychosis. The researchers aimed to capture a range of interrelated attitudes and perceptions of individuals in recovery from a first psychotic episode. The first step in generation of items was conducting 20 qualitative interviews with clients of a single clinic who were recovering from a first episode of psychosis. Questions posed were openended and were designed to encourage reflection on aspects of the illness experience individuals regarded as significant. Transcriptions of interviews were analyzed for themes. Potential items identified from the themes were formed into statements. The 
pool of items was then screened by two clinicians and a draft instrument was piloted to clients whose feedback was elicited. Both positively and negatively worded items were included on the first draft of the instrument. As respondents expressed confusion regarding the negative wording, only positive wording was used on the subsequent draft of the measure.

The version of the PRI that was subjected to a validation study consisted of 25 core items phrased as declarative sentences. A 6-point Likert scale was used that ranged from $1=$ "strongly disagree" to $6=$ "strongly agree." The study included 48 outpatient clients. A factor analysis found that the "attitude to illness" domain had three factors, the "attitude to treatment" domain had five factors, and the "perception of recovery and relapse" domain had two factors. The validation study included two concurrent measures to assess convergence, the Scale to Assess Unawareness of Mental Disorder (SUMD; Amador \& Strauss, 1993) and the Drug Attitude Inventory (DAI; Hogan \& Awad, 2000). The PRI and SUMD were modestly correlated $(r=0.28)$ as were the PRI treatment domain subscale and the DAI. The Cronbach's alpha coefficient test for internal consistency over the entire scale was 0.79 .

The researchers did not claim to have lived experience with psychosis and did not claim to have consumer advisors or consultants. However, the content of the instrument was drawn from qualitative interviews with individuals with lived experience. The instrument was developed as a self-administered questionnaire. As all items are positively worded and the instrument has standard response options, the instrument appears have a high ease of use. Some items feature a negative frame on experiences. 
For example, items read: "My illness has had a major adverse impact on my life" ; "I would have to fully return to how I was before I was ill before I would consider myself to have recovered from my illness" ; and "If I relapse, it would be disastrous for me." Main criticisms of this measure are the inclusion of only participants in early recovery from first-episode psychosis, low level of consumer involvement, and the negative frame of some items.

The Schizophrenia Hope Scale (SHS-9; Choe, 2013) was developed in South Korea and designed to measure hope in individuals with schizophrenia. Scale items were selected from literature reviews and were evaluated by an expert panel. The study included a total of 347 individuals with diagnoses of schizophrenia, schizoaffective, or schizophrenia spectrum disorders in care with three psychiatric hospitals and two community mental health centers. The 40-item instrument with a 3-point Likert scale which ranged from $0=$ "disagree" to $2=$ "strongly agree," was subjected to a validation study that included two concurrent measures to test for convergence, divergence, and both exploratory and confirmatory factor analyses. Convergent validity was established by a positive correlation with the State-Trait Hope Inventory (Farran, Herth, \& Popovich, $1995 ; r=0.61$ ) and divergent validity was established by a negative correlation with the Beck Hopelessness Scale (Beck, Weissman, Lester, \& Trexler, 1974; $r=-0.55$ ). Cronbach's alpha for the preliminary scale was 0.97 and for the five factors ranged from 0.84 to 0.96 . The second version of the instrument consisted of the 17 items that had loaded on the first factor, the essential meaning of hope. It was subjected to an exploratory factor analysis and had a Cronbach's alpha of 0.94. A 14-item instrument 
was then constructed and subjected to a confirmatory factor analysis. The resulting instrument with a Cronbach's alpha of 0.92 consists of 9 items that address the emotional and spiritual meanings of hope. The instrument has the original 3-point Likert scale. The range of possible scores is 1-18 with higher scores indicating higher levels of hope. The scale reflects the meaning of hope as consisting of positive expectations for the future, confidence in life and the future, and meaning in life.

The researcher does not claim lived experience and did not claim to have consumer advisors or consultants. Level of consumer input was low as the instrument was developed from review of the literature and from guidance of an expert panel that did not include individuals with lived experience. As the content of the scale does appear to speak to meaning in life and a self-direction, it appears to be consistent with recovery values. All items are positively worded and have a positive frame. The instrument is designed to be self-administered and both the brevity of the scale and the consistency of response options lend to ease of completion. An English translation of the scale is available but has not been psychometrically tested. Main criticisms of this instrument as a recovery measure include the sole focus on hope, the lack of consumer involvement, and the lack of psychometric testing in English-speaking countries.

The Maryland Assessment of Recovery in People with Serious Mental Illness (MARS; (Drapalski et al., 012) was developed in the U.S.A. based on the SAMHSA definition of recovery. Mental health recovery is defined as a journey of healing and transformation that enables a person with a mental health disability to live a meaningful life in communities of his or her choice while striving to achieve full human potential or 
personhood. SAMHSA identifies ten characteristics of recovery and recovery-oriented services: self-direction, individualized or person-centered empowerment, holistic, nonlinear, strength-based, peer support, respect, responsibility, and hope. The researchers critique the SAMHSA definition as providing dimensions of recovery rather than an operational definition. They used it as a guide for measure development and removed the domains that focused on service system, community, peer support, empowerment, and respect in order to focus on the internal factors of a consumer's recovery. Items were developed in consultation with a panel of experts that included consumers and with a consumer advisory panel. The resulting instrument consisted of 67 statements rated on a 5-point Likert scale that ranged from $1=$ "not at all" to $5=$ "very much." The scale was written at a fourth grade reading level. The instrument was evaluated using a total of 166 participants in two samples. Participants had diagnoses of schizophrenia, schizoaffective disorder, bipolar I, or major depression with psychotic features. After validation procedures, the resulting instrument consisted of 25 items. Scores range from 25 to 125 with higher scores indicative of higher levels of recovery. The MARS had good internal consistency with a Cronbach alpha of 0.95 . Test-retest reliability was good $(r=.898)$. A confirmatory factor analysis resulted in a 25 -item unidimensional scale. No validity information was made available. No information on criterion or construct validity was made available.

Level of consumer input for the development of the MARS was moderate. There were individuals with lived experience on the expert panel and the study included a consumer advisory panel. The self-administered scale is relatively brief with 25 items 
and the consistency of response options lends itself to ease of completion. All items are positively worded and reflect a positive frame on recovery. Three sample items are "I can influence important issues in my life," "I am hopeful about the future," and "Overcoming challenges helps me to learn and grow." The main criticisms of this measure include the only moderate level of consumer involvement and the lack of criterion and construct validity testing.

The Socially Valued Role Classification Scale (SRCS; Lloyd, Waghorn, Best, \& Gemmell, 2008) was developed in Australia as an outcome measure for psychosocial rehabilitation programs. The researchers defined recovery as a personal post-illness journey where active participation in the community is an accepted indicator. They expressed dissatisfaction with available social functioning measures that provided global ratings rather than addressing specific domains of role functioning. Social inclusion was conceptualized as multidimensional and represented by socially-valued role functioning, social support, stigma experiences, integration in the immediate psychosocial rehabilitation community, and integration in the wider neighborhood community. Items were generated through 26 interviews with members of a psychiatric psychosocial rehabilitation service known as a clubhouse. Participants self-reported diagnoses of bipolar disorder, schizophrenia, schizoaffective disorder, major depression with psychosis, delusional disorder, dissociative identity disorder, major depression, generalized anxiety disorder, and panic disorder. Participants were also administered the Community Integration Questionnaire (CIQ; Dijkers, 2000) to assess the extent of community integration within both the immediate psychosocial rehabilitation community 
and the wider neighborhood. All items are stated as declarative statements and are rated on a 5-point Likert scale with response options ranging from "always agree" to "always disagree." After testing for internal consistency, the measure consisted of 20 items.

A reliability and validity test of the SCRC (Harris et al., 2011) involved administration of the test by telephone to 60 community residents with schizophrenia or schizoaffective disorder. Participants were also administered the Work-related Self-efficacy Scale (Waghorn, Chant, \& King, 2005a), the Education-related Self-efficacy Scale (Harris et al., 2011), the Activity and Participation Questionnaire (Stewart et al., 2010), and the Work-related Subjective Experiences scale (Waghorn, Chant, \& King, 2005b). Concurrent validity was supported by moderate to very good associations between the SCRC and other measures. Test-retest reliability was good to very good.

The researchers did not claim to have lived experience and did not claim to have consumer advisors or consultants. As the measure was developed through interviews with individuals in recovery, the level of input for the development of the SCRC was moderate. The content of the scale items appears to reflect experiences that are important to individuals in recovery. Sample items include, "I feel like a part of this neighborhood, like I belong here," and "I feel that I am accepted by people in this Clubhouse or Social Group.” All items are positively worded and reflect a positive frame on recovery. The instrument has consistent response options and appears to have ease of administration. It is not designed for self-administration, however. The SCRC appears to be under development and to be unaligned with consumer recommended instrument development 
procedures. Main criticisms of this measure include the lack of consumer involvement, that it is not designed for self-administration, and the need for more psychometric testing.

The Mental Health Statistics Improvement Program (MHSIP) Adult Consumer Survey (Jerrell, 2006) was developed in the USA to evaluate the performance of a mental health system in the domains of access, quality, and appropriateness of services and outcomes from the perspective of the consumer. Use of the MHSIP is mandated for Block Grant funding from the federal government to the states. The Likert response formatted 56item instrument had previously been found to have five factors: participation in treatment planning, treatment leads to recovery, staff helpful and trustworthy, perceived limitations caused by illness, and relationship with doctor. (Ganju, 1999). This examination of the measure used a sample of 850 clients of 17 mental health centers in South Carolina. The instrument was administered by seven consumers of mental health services who had previous involvement in conducting research. The Consumer to Consumer Evaluation Team (CCET) Questionnaire was also administered to evaluate convergent validity. The instrument was subjected to a factor analysis using principal components and varimax rotation. Three factors were identified representing 16 items: access, quality and appropriateness, and perceived outcomes. Cronbach's alpha coefficient was good for all subscales: .83 for access; .88 for quality and appropriateness; and .77 for perceived outcomes Test-retest reliability was moderate, ranging from 0.45 to 0.61 . Convergent validity correlations were good.

This examination of the MHSIP involved consumers trained to conduct research and so appears consistent with recommended research practices developed in partnership with 
individuals with lived experience. The items appear to reflect issues identified as significant by consumers such as accessible service, self-direction, and social supports. Items are positively worded and reflect a positive frame on recovery. However, the MHSIP is limited as a recovery measure as it focuses largely on quality of services and is not designed for self-administration.

A Measure of Social Health (Carlson et al., 2011) was developed in the USA to focus on social health as no validated, published measures could be found. The concept of social health focuses on social activities, well-being, social network and supports, interpersonal communication, and satisfaction with social role participation. Social functioning is an important aspect of one's overall social health and refers to an individual's ability to function in community, social, and occupational domains. Measures of social functioning have been used in social health assessment. The researchers evaluated a set of social health scales derived from items on two widely used mental health recovery instruments: the California Quality of Life (CA-QOL; California Department of Mental Health, 1999) and the Mental Health Statistics Improvement Program Consumer Survey (MHSIP; Jerrell, 2006). Neither the CA-QOL nor the MHSIP were developed with a sole focus on social health. They compared the psychometric properties of the identified CA-QOL Social Health Scale and MHSIP Social Health Scale with the established Social Functioning Questionnaire (SFQ); (Tyrer et al., 2005). The researchers identified a total of 8 items from the CA-QOL and MHSIP based on conceptual correspondence with the social health construct. Psychometric evaluation found that Pearson correlations with the SFQ and the CA-QOL ranged from 0.48 to 0.62 
and with the MHSIP ranged from 0.42 to 0.50 . The researchers concluded that the identified social health scales demonstrated moderate to strong relationships with the validated SFQ but did not provide a statistical measure of internal reliability. They conclude that the CA-QOL and/or MHSIP items can be used to assess social health for research and assessment purposes when use of a separate social health measure is not feasible given the time and financial constraints found in the field.

The researchers did not claim to have lived experience with mental health challenges and did not claim to have involved consumer consultants or a consumer advisory council in the study. However, this was a secondary data analysis study that consisted only of statistical procedures. The items of the social health scale are declarative statements that are positively worded and reflect a positive frame on recovery. The proposed scale would use consistent Likert response options and would appear to feature both brevity and ease of self-administration. Main criticisms of the Measure of Social Health as a recovery measure include the lack of consumer involvement. Further, the developers emphasized that the measure was intended to be used in conjunction with other measures of recovery.

A Social Inclusion Questionnaire (Marino-Francis \& Worrall-Davies, 2010) was developed in the United Kingdom to be used by community mental health services as an outcome tool. The developers of the instrument aimed to design and validate a measure that both reflected the local definition of social inclusion and was relevant to the views of service users. A literature search on social inclusion found no commonly accepted definition. The researchers brought the number of definitions of social inclusion to mental health administrators and service users for review. The following definition was 
derived: Social inclusion is about each person taking part in society and having control over their own resources. It is also about a community that cares for its members, makes them feel welcome, and is willing to adjust to fit their various needs. The following measures were selected for review in order to identify key areas of social inclusion: Evaluating Social Inclusion for People Who Use Mental Health Services (Stickley \& Shaw, 2006); the Support Needs Questionnaire (Davis \& Burns, 2015); the Inclusion Web (Bates, 2006); Measure for Social Inclusion for Arts and Mental Health Project Participants (Secker et al., 2009); Camberwell Assessment of Need Short Appraisal Schedule (Slade et al., 1999); Social Capital/Social Exclusion Condensed Module (Bajekal \& Purdon, 2002); and the Poverty and Social Exclusion Survey of Britain Questionnaire (Gordon et al., 1999). The researchers created 23 items with a 5-point Likert response format that ranged from $1=$ "not at all" to $5=$ "all the time." Lower scores were indicative of lower social inclusion. One item was reverse scored. Five focus groups were held in consultation with mental health staff and service users and revisions were made to the wording of items. A validation test was conducted with a sample of 69 mental health day service users in one metro area of England. Cronbach's alpha was .80 and reliability testing resulted in Spearman's Rho coefficient values between .312 and .820. A factor analysis using principal component analysis and varimax rotation led to the removal of three items that cross loaded and resulted in a 7 factor model.

The researchers did not claim to have lived experience with recovery but did claim to make use of consumer advisors. The level of input for the development of the instrument could be said to be moderate. The content of the scale appears reflective of aspects of 
recovery important to consumers. Sample items include, "I have felt accepted by my friends," and "I have felt part of my community." Only one item had negative wording and the instrument as a whole reflected a positive frame on recovery. The selfadministered instrument would appear to feature ease of use as it is brief and response options are consistent. Main criticisms of the Social Inclusion Questionnaire include the only moderate level of consumer involvement and the stated aim to develop a measure that reflected a local definition of social inclusion.

The Social Inclusion Questionnaire User Experience (SlnQUE; Mezey et al., 2012) was developed in England to measure the social inclusion of individuals with schizophrenia and schizoaffective disorders. The researchers stated that they could find no generally accepted or validated measure of social inclusion for such a population. They based the measure on the Poverty and Social Exclusion Survey that had been administered to the general population in Britain (Gordon et al., 1999). The researchers acknowledged that the relevance of the survey to individuals with severe mental illness was unknown. The survey identified four domains pertaining to social inclusion: productivity, consumption, access to services, and social relations. Items were drawn from the domains with some additional questions that focused on political engagement. Items were designed to address the extent to which such individuals are socially included, their desire for social inclusion, and changes in social inclusion. The instrument had two parts, the first pertaining to the year prior to hospitalization and the second to their current situation. The SInQUE was designed as a structured interview rather than a selfreport measure and a dichotomous yes/no response format was utilized. A higher score 
indicates greater social inclusion. A validation test was conducted with a sample of 66 individuals diagnosed with schizophrenia or schizoaffective disorder who were clients of community mental health agencies in London. Convergent and discriminant validity was assessed by administrating the Manchester Short Assessment of Quality of Life (MANSA); (Priebe et al., 1999), the Camberwell Assessment of Needs Short Appraisal Schedule (CANSAS); (Slade et al., 1999), and the Brief Psychiatric Rating Scale (BPRS); (Ventura et al., 1993). Concurrent validity was assessed by calculating social outcome index (SIX) scores from the SInQUE items that correlated to the domains of the SIX. Concurrent validity was established but convergent and divergent validity testing found that only the domains of current social integration, productivity, and social integration demonstrated expected correlations. Reliability testing was not conducted.

The researchers did not claim lived experience with recovery and did not claim to involve consumer consultants or an advisory council. Items were developed from a social inclusion survey administered to a general population. The applicability of the evidence to the population of individuals with diagnoses of serious mental illness is debatable. The content of the instrument did not appear to address such recovery values as growth through adversity or self-determination. Items were not positively worded and did not reflect a positive frame on recovery. The instrument was not designed to be selfadministered and required 45 minutes for completion. Main criticisms of the SInQUE include the apparent lack of consumer involvement, hat it was based on a survey designed for the general population, and that items did not reflect consumer identified recovery values. 
The Individual Recovery Outcomes Counter (IROC; Monger, Hardie, Ion, Cumming, \& Henderson, 2013) was developed in Scotland to measure recovery. The researchers conceptualized recovery as both an outcome and a process that is inherently subjective. They view recovery concepts as fluid and as inclusive of both resolution of symptoms and of valuing the illness experience and reclaiming social roles and citizenship. A draft measure was developed by managers of mental health agencies by drawing on experience and examining existing measures. Wording was changed based on feedback from a group of 40 service users. Focus groups with service users and staff were then held and more wording changes were made. A 12 -item scale using three-option response indicators was subjected to validity testing. The 171 participants were recipients of community mental health services in one city. Convergent validity was examined by administering the Recovery Scale (RAS; Giffort, Schmook, Vollendorf, \& Gervain, 1999) and the Behavior and Symptom Identification Scale (BASIS-32; Eisen, 1995) and expected correlations were found. The IROC had good internal consistency of 0.86 . An exploratory factor analysis using a varimax rotation indicated two underlying factors, interpersonal such as skills and self-worth and intrapersonal such as social network and hope.

The researchers did not claim to have lived experience with recovery. The initial draft of the measure was constructed by mental health system managers based on professional experience and a review of existing measures. However, service user input led to refinement of the instrument. Level of consumer input could be said to be moderate. The content of the scale appeared to reflect aspects of recovery identified as relevant by 
consumers such as social supports and hope. All items were positively worded and reflective of a positive frame on recovery. The self-administered scale is brief at 12 items and the response categories are consistent. Main criticisms of the measure include that it may best pertain to Scottish recovery values and that the level of consumer involvement was only moderate.

Functioning Assessment Short Test (FAST; Rosa et al., 2007) was developed in Spain in order to assess specific levels of psychosocial functioning for individuals with serious mental illness, particularly individuals with bipolar disorder. The researchers noted that while many individuals with bipolar disorder experienced symptomatic recovery, only a minority achieved functional recovery as conceived as ability to work, pursue education, live independently, and engage in partnership and other significant relationships. They found that existing measures focused on only one or two elements of functioning and that none of the measures were developed to assess individuals with bipolar disorder. The initial version of the instrument included 56 items divided into the 10 areas of autonomy, work functioning, cognitive functioning, finances, insight, social/marital life, acceptance/knowledge of disorder, strategies to cope with symptoms, use of medication, and self-fulfillment. After conducting a pilot study and consultation with experts, they created a 24-item measure that was divided into the 6 specific areas: autonomy, occupational functioning, cognitive functioning, financial issues, interpersonal relationships, and leisure time. Items are rated on a 4-point Likert scale with responses that range from $0=$ "no difficulty" to $3=$ "severe difficulty." Higher scores indicate more serious difficulties. Validity testing was conducted with a sample of 100 individuals 
diagnosed with bipolar disorder who were clients of a single Spanish mental health clinic. The internal consistency coefficient of the total scale was high as measured by a Cronbach's alpha of 0.909. There was high internal consistency on each of the items as well. Concurrent validity with the GAF (APA, 2013) was highly significant $(r=0.903)$. Higher scores on the GAF represent better psychosocial functioning, whereas higher scores on the FAST represent higher disability. Test-retest reliability was high. Examination of the internal structure of the instrument using varimax rotation found a five factor structure.

The researchers did not claim to have lived experience and did not claim to have consumer advisors or consultants. The instrument was developed from a review of the literature and from consultation with experts. A notable strength is that content of the scale does appear to reflect aspects of recovery consumers have identified as important such as social and work roles and autonomy. Some items were negatively worded and the frame of the instrument was negative as it assesses for disability. The self-administered measure appeared to have ease of completion as it was brief and response categories were consistent. The FAST may be limited as a recovery measure as it was designed with a particular focus on individuals with bipolar disorder and as it was developed without consumer involvement.

The Social Functioning Questionnaire (SFQ; Tyrer et al., 2005) was developed in England as a test of social functioning for individuals with primarily non-psychotic mental health challenges. The researchers conceive of psychiatric disability as caused by poor social functioning at least as much as by symptoms. They explore how some 
individuals with significant impairments can have excellent social functioning and good quality of life. The 8-item instrument was developed as a self-rated equivalent of the Social Functioning Schedule (SFS; Birchwood, Cochrane, Wetton, \& Copestake, 1990), a semi-structured interview covering 14 domains of function rated on visual analogue scales. The SFQ items are phrased as declarative statements and are rated with a 4-point Likert scale. Response options range from 0 to 3 . The options differ from question to question. Some options are oriented to frequency and some to severity of difficulties. Possible total scores range from 0-24 with higher scores indicative of worse functioning. The instrument has been used in a number of clinical studies, including one with individuals with psychosis, and a national epidemiological study. Over 4000 individuals participated in the studies as subjects. The researchers do not provide the specific results of psychometric tests but claim: high internal consistency; strong test-retest reliability; and strong convergent validity with the Comprehensive Psychopathological Rating Scale (Asberg \& Schalling, 1979).

The developers of the SFQ did not claim to have lived experience with recovery and no study claimed to have made use of consumer consultants or advisors. The content of items does appear to reflect aspects of recovery identified as important to consumers such as occupation of work and social roles. Some items are negatively worded, e.g., "I have difficulty in getting and keeping close relationships." The SFQ takes four minutes to complete. However, response options differ from question to question which may make the instrument challenging for some respondents. The SFQ may be limited as a measure 
of recovery as it was developed primarily for individuals who have not experienced psychosis and as no consumers were involved in its development.

The Integrative Hope Scale (IHS; Schrank et al., 2011) was developed in Austria to measure hope for individuals diagnosed with severe mental illnesses. The researchers conceive of recovery as a process and hope as a central factor associated with well-being, resilience, adaptation, and therapeutic change. They review the literature on the large number of overlapping but non-equivalent definitions of hope with their corresponding scales. Further, they explore the lack of understanding of the specific mechanisms by which hope operates in recovery. They did not find any scales of hope that were developed for individuals with severe mental illness, only subscales of existing measures such as the Recovery Assessment Scale (RAS; Giffort, Schmook, Vollendorf, \& Gervain, 1999). The Integrative Hope Scale (IHS) was developed from this scale and literature review. Items are worded as declarative statements and are rated with a 6-point Likert scale. Response options range from $1=$ "strongly disagree" to $6=$ "strongly agree." Possible total scores range from 22 to 132 with higher scores indicative of greater hope.

The validation study used a sample of 200 individuals diagnosed with schizophrenia and schizoaffective disorder who were clients of community mental health services in the city of Vienna, Austria. Concurrent validity was measured by the use of the Center for Epidemiological Studies Depression Scale (CES-D; Eaton, Muntaner, Smith, Tien, \& Ybarra, 2004), the Positive and Negative Syndrome Scale (PANSS; Kay, Opler, \& Fiszbein, 1987), and the Client Socio-demographic and Service Receipt Inventory (CSSRI-EU; Knapp, Martin, \& Chisholm, 2006). A confirmatory factor analysis found a 
four factor structure: trust and confidence, positive future orientation, social relations and personal value, and lack of perspective. The lack of perspective factor included items reflective of the cognitive distortion of polarized or black and white thinking. One item was removed resulting in a final instrument of 22 items. Internal consistency as measured by Cronbach's alpha was 0.92 for the total scale and consisted of a range of 0.74 to 0.94 for the different factors. Test-retest reliability was 0.84 . Discriminant validity was demonstrated by a significant negative correlation $(r=-.58)$ with the depression measure and with the negative symptoms of the PANSS.

The researchers did not claim to have lived experience with recovery and did not claim to make use of consumer consultants or advisors. The content of the items appears to largely reflect aspects of recovery identified as important by consumers such as social supports, growth through adversity, and a goal orientation. However, the lack of perspective factor contained items that reflected a negative frame on recovery. Two examples are, "I feel trapped, pinned down," and "I am bothered by troubles that prevent my planning for the future.” The self-administered instrument appears to have ease of use as response options are consistent and the scale is brief. The HIS may be limited as a recovery measure given the sole focus on hope and due to the lack of consumer involvement in its development.

My Voice, My Life (MVML; Gordon, Ellis, Siegert, \& Walkey, 2013) was developed in New Zealand as a consumer recovery outcome measure. The consumer-led research team worked with a 15-member consumer reference group throughout the instrument development process. They conceptualized recovery as involving social and other life 
skills, involvement in one's culture, physical health, the ability to cope with emotions and mental distress, quality of life, hope, empowerment, spirituality, access to materials to meet basic needs, and satisfaction with services. After a review of the literature and consultation with consumers, they hypothesized that that 12 domains underpinned the construct of consumer recovery and drafted a preliminary measure that consisted of 127 items made up of 5-20 items for each domain. Items were phrased as declarative statements that referenced the past week. Items were rated with a 5-point Likert scale with response options that ranged from 1 = "a little of the time" to $4=$ "all of the time" and $5=$ "not applicable." The preliminary measure was administered to 511 individuals who self-identified as having experience with mental illness. The individuals were recruited through mental health services, consumer organizations and networks, and mental health conferences. An exploratory factor analysis was conducted with half the sample data and the refined measure was reviewed by the consumer group. A confirmatory factor analysis was then conducted with the remaining half of the sample data. The analysis supported an 11 factor model. Hope and empowerment were combined into a single domain. All items with factor loadings below 0.70 were dropped which resulted in a 65-item scale. Internal consistency was high with a Cronbach's alpha of 0.94 for the total scale and a range of 0.80 to 0.96 for each subscale. Convergent/divergent validity and test-retest reliability testing were not conducted.

The measure was developed by a research team led by an individual who identified as having lived experience with recovery and in partnership with a consumer reference group. The level of consumer involvement is therefore high. Items appear to reflect 
aspects of recovery considered important by consumers such as trusting relationships and self-determination. All items are phrased in a positive manner and the overall instrument reflects a positive frame on recovery. Response options are consistent throughout the measure. The self-administered instrument is long at 65 items which may pose a challenge to some individuals for whom the instrument was apparently designed. Main criticisms of the measure include its great length and the lack of psychometric testing outside of New Zealand.

The Multidimensional Scale of Independent Functioning (MSIF; Jaeger, Berns, \& Czobor, 2003) was developed in the USA to rate functional disability in psychiatric outpatients, in particular individuals with diagnoses of schizophrenia, schizoaffective disorder, and major affective disorders. The researchers framed recovery as involving independent functioning more than just symptom levels alone. They reviewed the only modest correlations that have been found between symptom levels and functional disability as well as how some instruments confound symptom severity and disability. An effective instrument would measure independent functioning free from psychopathology. The MSIF was designed to discriminate between the dimensions of role responsibility, support, and performance within work, education, and residential domains. It is administered through a semi-structured interview by a clinician. Each dimension is rated along a 7-point Likert scale with options that range from $1=$ "normal functioning" to $7=$ "total disability." A validation study used a sample of 114 individuals in outpatient care. Convergent validity was tested by administration of the Social Adjustment Scale II (SAS II; Weissman, 1999). The two measures demonstrated a significant positive association. 
Internal consistency was moderate with a Cronbach's alpha of 0.70 and inter-rater reliability ranged from 0.74 to 1.00 for global and subscale scores.

The developers of the MSIF did not claim to have lived experience with recovery and did not claim to make use of consumer consultants or advisors. The focus on independent functioning as opposed to symptoms appears consistent with consumer views on recovery as does the focus on the context of functioning. In general, the content of the instrument reflects a positive frame on recovery. The instrument is not self-administered but has a consistent format which lends itself to ease of completion. Main criticisms of the measure include the apparent lack of consumer involvement and the focus on functioning as opposed to self-determination and involvement in meaningful activity.

Table 1. Summary of Instrument Quality Ratings

\begin{tabular}{|l|c|c|c|c|c|}
\hline & $\begin{array}{l}\text { Psychometric } \\
\text { Robustness }\end{array}$ & $\begin{array}{l}\text { Consumer } \\
\text { Involvement }\end{array}$ & $\begin{array}{l}\text { Relevancy } \\
\text { to } \\
\text { Recovery }\end{array}$ & $\begin{array}{l}\text { Positive } \\
\text { Framing }\end{array}$ & $\begin{array}{l}\text { Ease of } \\
\text { Administration }\end{array}$ \\
\hline $\begin{array}{l}\text { The Stages of } \\
\text { Recovery } \\
\text { Instrument } \\
\text { (STORI) }\end{array}$ & + & - & + & - & - \\
\hline $\begin{array}{l}\text { The Psychosis } \\
\text { Recovery } \\
\text { Inventory } \\
\text { (PRI) }\end{array}$ & + & - & + & + & + \\
\hline $\begin{array}{l}\text { The } \\
\text { Schizophrenia }\end{array}$ & + & - & + & ++ & + \\
$\begin{array}{l}\text { Hope Scale } \\
\text { (SHS-9) }\end{array}$ & & & & & \\
\hline $\begin{array}{l}\text { The Maryland } \\
\text { Assessment of } \\
\text { Recovery in } \\
\text { People with } \\
\text { Serious } \\
\text { Mental Illness } \\
\text { (MARS) }\end{array}$ & + & + & + & + & ++ \\
\hline
\end{tabular}




\begin{tabular}{|c|c|c|c|c|c|}
\hline & $\begin{array}{c}\text { Psychometric } \\
\text { Robustness }\end{array}$ & $\begin{array}{c}\text { Consumer } \\
\text { Involvement }\end{array}$ & $\begin{array}{c}\text { Relevancy } \\
\text { to } \\
\text { Recovery }\end{array}$ & $\begin{array}{l}\text { Positive } \\
\text { Framing }\end{array}$ & $\begin{array}{c}\text { Ease of } \\
\text { Administration }\end{array}$ \\
\hline $\begin{array}{l}\text { Socially } \\
\text { Valued Role } \\
\text { Classification }\end{array}$ & + & + & + & ++ & - \\
\hline $\begin{array}{l}\text { The Mental } \\
\text { Health } \\
\text { Statistics } \\
\text { Improvement } \\
\text { Program } \\
\text { (MHSIP) }\end{array}$ & + & + & + & ++ & + \\
\hline $\begin{array}{l}\text { A Measure of } \\
\text { Social Health }\end{array}$ & + & - & + & ++ & + \\
\hline $\begin{array}{l}\text { A Social } \\
\text { Inclusion } \\
\text { Questionnaire }\end{array}$ & + & + & + & + & + \\
\hline $\begin{array}{l}\text { The } \\
\text { Individual } \\
\text { Recovery } \\
\text { Outcomes } \\
\text { Counter } \\
\text { (IROC) }\end{array}$ & + & + & + & ++ & ++ \\
\hline $\begin{array}{l}\text { Functioning } \\
\text { Assessment } \\
\text { Short Test } \\
\text { (FAST) }\end{array}$ & ++ & - & + & - & + \\
\hline $\begin{array}{l}\text { The Social } \\
\text { Functioning } \\
\text { Questionnaire } \\
\text { (SFQ) }\end{array}$ & ++ & - & + & + & - \\
\hline $\begin{array}{l}\text { The } \\
\text { Integrative } \\
\text { Hope Scale }\end{array}$ & + & - & + & + & + \\
\hline $\begin{array}{l}\text { My Voice, } \\
\text { My Life } \\
\text { (MVML) }\end{array}$ & + & ++ & ++ & ++ & + \\
\hline
\end{tabular}




\begin{tabular}{|l|c|c|c|c|c|}
\hline & $\begin{array}{c}\text { Psychometric } \\
\text { Robustness }\end{array}$ & $\begin{array}{c}\text { Consumer } \\
\text { Involvement }\end{array}$ & $\begin{array}{c}\text { Relevancy } \\
\text { to } \\
\text { Recovery }\end{array}$ & $\begin{array}{c}\text { Positive } \\
\text { Framing }\end{array}$ & $\begin{array}{c}\text { Ease of } \\
\text { Administration }\end{array}$ \\
\hline $\begin{array}{l}\text { The } \\
\text { Multidimensi } \\
\text { onal Scale of } \\
\text { Independent }\end{array}$ & + & - & + & + & + \\
$\begin{array}{l}\text { Functioning } \\
\text { (MSIF) }\end{array}$ & & - & - & - & \\
\hline $\begin{array}{l}\text { The Social } \\
\text { Inclusion } \\
\text { Questionnaire }\end{array}$ & - & & & & \\
$\begin{array}{l}\text { User } \\
\text { Experience } \\
\text { (SInQUE) }\end{array}$ & & & & & \\
\hline
\end{tabular}

Note: $-=$ does not meet evaluation criteria $;+=$ meets some evaluation criteria $;++=$ meets all evaluation criteria

\section{Discussion of Instruments}

Recovery challenges measurement due to the multitude of definitions of the concept. It has been conceptualized as an iterative process, a stage phenomenon, and an outcome. Different weights are given to symptomatology, functioning, identity issues, personal growth, and social inclusion. Hope appears central to the recovery process and at times appears to be confounded with recovery. The determinants of recovery appear to be an ongoing subject of dispute. There is little agreement to be found regarding the particular mediators and moderators of recovery.

This brief review of relevant measures presented snapshots of a landscape containing a variety of theory bases and overlapping constructs. The review found a continuum of apparent consumer input that ranged from nonexistent to consumer control of the entire process. Additionally, recovery instrument development clearly varied in terms of psychometric rigor. Even the best of the instruments reviewed could be improved in 
terms of rigor and/or ease of use. It may be that recovery consists of a number of relatively independent domains. As a multifaceted concept, multiple measures may be required.

No measure had been found to date that was grounded in the social model of disability or mad theory bases and no measure was specifically addressed to social recovery. Social recovery involves the experience of meaningful relationships, a sense of being able to make a social contribution, and a sense of belonging (Slade, 2010; Whitley \& Drake, 2010). The recovery process involves introduction of an individual into an accepting environment (Secker, Membrey, Grove, \& Seebohm, 2010). While The Maryland Assessment of Recovery in People with Serious Mental Illness (MARS) had certain strengths as an instrument, it was developed with only a moderate level of consumer involvement and had a lack of construct validity testing. The Social Inclusion Questionnaire (SIQ) also had notable strengths but focused on control over resources while living in a caring community instead of focusing more on individuals having a strong self-concept and a sense that they could contribute. Development of the Social Recovery Measure was intended to address this gap in the recovery measurement field. The measure was developed in partnership with individuals with lived experience and with great attention to psychometric properties.

Recovery science will only advance with reliable measurement (Bellack \& Drapalski, 2012; Liberman \& Kopelowicz, 2005). It would seem that the multidimensional nature of recovery calls for the use of multidimensional measurement (Jaaskelainen et al., 2012). Given the unique characteristics and experiences of individuals in recovery, a single ideal 
instrument may not be necessary and may even run counter to the diversity of journeys of recovery (Scheyett, DeLuca, \& Morgan, 2013). The development of a Social Recovery Measure was needed to specifically address the social processes of recovery. 


\section{Chapter 3}

\section{METHODOLOGY}

The purpose of this study was to develop a measure of social recovery and establish the reliability and validity of the measure. Both psychiatric disability and recovery are beginning to be explored as inherently social processes. Such exploration requires the measurement of the social aspects of recovery. While there has been a call for improvement of existing recovery instruments by the addition of functional and social domains (Hancock, Bundy, Honey, Helich, \& Tamsett, 2013), there was no measure specific to social recovery to be found in the peer reviewed literature (Carlson et al., 2011). The Social Recovery Measure is grounded in disability and mad theory and specifically targets recovery as a social process. This chapter reviews the instrument development process, methodological rationale, procedures, statistical analyses used to develop and test the measure, and general guidelines of implementation.

The process of developing the Social Recovery Measure was primarily guided by the work of Robert F. DeVellis (2012) and by Neill Abell, David Springer, and Akihito Kamata (2009) as they are widely recognized as experts in the field who provide systematic approaches to instrument development. Additionally, Drs. Abell and Springer are both social work academics. DeVellis provides an eight-step framework for developing and validating a measurement instrument that Abell, Springer, and Kamata endorse. The guidelines are as follows:

1. Determine clearly what you want to measure

2. Generate an item pool. 
3. Determine the format for measurement.

4. Have the initial item pool reviewed by experts.

5. Consider inclusion of validation items

6. Administer items to a development sample

7. Evaluate the items

8. Optimize scale length.

Chapter 2 of this dissertation established step 1, what is to be measured. This chapter continues with work on step two. The drafts of the Social Recovery Measure will be presented along with an explanation of how they were developed. The procedures for further refinement and for item evaluation and psychometric testing will be reviewed. Before exploring these matters, general guidance on instrument development will be reviewed.

\section{Instrument Development}

Instruments are developed when we want to measure phenomena that we theorize exist but that we cannot assess directly. DeVellis (2012) has emphasized that clarity of thought regarding the construct being measured is required for clarity of thought about the content of a scale. In measurement theory, the construct in scale development is understood as a latent variable that is not directly observable. A latent variable can be represented by information from more than one relevant observed variable. Observable indicators such as quantified responses to individual scale items can express the latent variable. Items are created in order to translate abstract ideas into specific representations 
of the construct. The latent variable is considered to be the cause of responses to individual scale items (Abell, Springer, \& Kamata, 2009).

It is generally understood that being well-grounded in a theory base will aid clarity of measurement. Unfortunately, a widely recognized challenge to the measurement of recovery is the lack of a common definition due to the number of theoretical perspectives held by different stakeholder groups. Not having a common definition of recovery is seen as interfering with the progress of research (Campbell-Orde, Chamberlin, Carpenter, \& Leff, 2005). As discussed in Chapter 2, the consumer/mad movements view recovery as a complex process that, while different for everyone, involves growth through adversity and community membership (Adame \& Knudson, 2007). Consumer/mad accounts of recovery contain common themes such as the importance of hope, empowerment, selfdetermination, and social support (Law, Morrison, Byrne, \& Hodson, 2012). While differences and experiences with impairment are not denied, there is a strong focus on the social aspects of recovery. The view is reflective of the disability and mad theory base which locate disability at the intersection of the person and the environment. Psychiatric disability is seen as involving both personal and political experiences and as inherently relational (Hopper, 2007; Loja, 2013; Mulvany, 2000).

As Chapter 2 notes, a significant criticism of many existing recovery instruments is the lack of input during development from individuals with lived experience of mental health challenges and recovery. Developing instruments in strong partnership with recovered individuals and by researchers with lived experience is becoming the new standard of practice (Campbell-Orde, Chamberlin, Carpenter, \& Leff, 2005). The Social 
Recovery instrument under development is aligned with such practice as I identify as in recovery from bipolar disorder and will create the measure with other individuals with lived experience of mental illness.

Drawing on the social model of disability and mad theory bases, I designed a qualitative study on the elements and processes of social reengagement in recovery. Approval was gained from the Portland State University Institutional Review Board Office of Research Integrity. Leaders of peer-delivered service and/or consumer advocacy programs in a metro area of the northwestern United States were contacted about the research project. Those who agreed to become involved recruited research participants from their respective programs. Five of the six leaders also engaged as research participants. Six focus groups were held with a total of 41 individuals with lived experience of mental illness and recovery. All of the participants were engaged in community mental health treatment and/or consumer advocacy at the time of the study. Groups were held at sites where participants engaged in services or leadership activities. Twenty participants identified as male, 20 as female, and one self-described as a spiritual being. They ranged in age from 21 to 69 years old and included 30 Caucasians and 11 individuals who were people of color. Reported diagnoses included schizophrenia, schizoaffective disorder, bipolar disorder, anxiety disorders, major depression, alcohol dependency in remission, and borderline personality disorder. Five individuals declined to provide a diagnosis. The groups took place between August 2013 and March 2014 and were recorded and transcribed by the researcher. The focus groups were semi-structured and the questions asked were: 
(1) Have you heard of the concept of social recovery? What does it mean to you?

(2) Would you tell me about your social recovery experiences and process?

(3) Do you know of someone who has a very good social recovery? What has made that person's social recovery so good?

The inductive processes of the grounded theory approach and constant comparative method were utilized (Glaser \& Strauss, 2009; Lincoln \& Guba, 1985). Each focus group was analyzed for units of meaning followed by analysis across focus groups. Material was grouped into meta-thematic and thematic categories. Participants spoke to the importance of a felt sense of belonging to a community in which they were valued. They required acceptance, safety, encouragement to grow, and relationships that featured authenticity and support. Social recovery involved a sense of inherent self-worth and environments in which people could be both vulnerable and explore their competency. No longer equating oneself with a mental health history and diagnosis was regarded as essential by many. Individuals needed to externalize the illness experience so as to build an identity beyond the illness and related labels. Participants also emphasized that social recovery involved a broad skills set inclusive of letting go of past difficulties and living in the present, practicing acceptance, maintaining a connection to their inner life and living authentically, and expressing oneself. They reported that they needed skills to manage distress and social situations. They emphasized the importance of having hope (Marino, 2015). Consistent with recommendations on recovery measure development offered by researchers with lived experience of madness (Law, Morrison, Byrne, \& Hodson, 2012), the scale items that were derived from the qualitative data were positively 
framed so as to speak to the presence of supports and capacities. The resulting first draft of a measure included the following items:

\section{Community}

1. I have a sense of belonging to a community that values me

2. I have a sense of safety in community

3. I have a community that encourages me to grow

4. I know people who accept me for who I really am

5. I have authentic relationships

6. I have people I can trust

7. I have relationships that are mutually supportive

8. I know people who can understand my experiences

9. I can make use of my abilities and make a contribution

10. I have meaningful things to do

11. I have access to the material resources I need

\section{Self-concept}

12. I am acceptable

13. I am not defined by my mental health challenges

14. I value my past experiences

15. I have a connection to my true self

16. I know what is true and right for me

17. I can be vulnerable

18. I am a worthy person 
19. I have something to offer others

20. I have inner motivation and can direct myself

21. I am responsible for myself

\section{Capacities}

22. I have let go of the past

23. I can ground to the present moment

24. I have skills to interact in society

25. I can express my inner experiences

26. I can be my authentic self

27. I can pursue my goals

28. I can connect with others

29. I can accept experiences even if they are difficult

30. I choose which relationships are right for me

31. I can cope with mental distress

32. I have hope for the future

As mentioned in Chapter 2, key recovery processes identified and understood to be measurable dimensions of change have become known by the acronym CHIME (Leamy, Bird, Le Boutillier, Williams, \& Slade, 2011). CHIME stands for: connectedness, hope, identity, meaning in life, and empowerment. As evaluated by the researcher, the items of the first draft of the Social Recovery measure as originally numbered can be placed in the CHIME categories as:

Category 1: Connectedness: support, relationships, community involvement 
1. I have a sense of belonging to a community that values me.

2. I have a sense of safety in community.

4. I know people who accept me for who I really am.

5. I have authentic relationships.

6. I have people I can trust.

8. I know people who can understand my experiences.

Category 2: Hope: belief in recovery, motivation, hope-inspiring relationships, goals

3. I have a community that encourages me to grow.

7. I have relationships that are mutually supportive.

13. I am not defined by my mental health challenges.

20. I have inner motivation and can direct myself.

22. I have let go of the past.

32. I have hope for the future.

Category 3: Identity: positive sense of self and identity, overcoming stigma

9. I can make use of my abilities and make a contribution.

12. I am acceptable.

15. I have a connection to my true self.

17. I can be vulnerable

18. I am a worthy person.

26. I can be my authentic self. 
Category 4: Meaning in life: meaning of mental illness experiences, quality of life, meaningful life and social roles, meaningful life and social goals, rebuilding a life

10. I have meaningful things to do.

11. I have access to the material resources I need.

14. I value my past experiences.

19. I have something to offer others.

27. I can pursue my goals.

28. I can connect with others.

30. I choose which relationships are right for me.

Category 5: Empowerment: personal responsibility, control over life, focus on strengths.

16. I know what is true and right for me.

21. I am responsible for myself.

23. I can ground to the present moment.

24. I have skills to interact in society.

25. I can express my inner experiences.

29. I can accept experiences even if they are difficult.

31. I can cope with mental distress.

\section{Expert Panel}

In an effort to determine if the proposed measure accurately represented social recovery, an expert panel was established of four individuals with experience with recovery and measurement. The panel members were: 
Larry Davidson, $\mathrm{PhD}$, is Professor of Psychology and director of the Program for Recovery and Community Health and the School of Medicine and Institution for Social and Policy Studies of Yale University. He directs the Recovery to Practice Initiative for SAMHSA and has researched recovery processes, peer-delivered services and program evaluation, and recovery supportive policy development. He has lived experience with psychiatric disability.

Nev Jones, $\mathrm{PhD}$, is Director of Research and Evaluation at the Felton Institute in San Francisco, California. Her research foci include psychosis, early intervention, the Hearing Voices movement, and the sociocultural determinants of illness and recovery. She has lived experience with psychiatric disability.

Stephanie Hayes, OTR, PhD candidate at University of California Berkeley School of Social Welfare, has a research focus on peer services and peer-driven organizations. She has lived experience with psychiatric disability.

Candice Morgan, $\mathrm{PhD}$ candidate at University of South Carolina, has a research focus on homelessness and mental health challenges. She was a co-investigator with Drs. Anna Scheyett and Joseph DeLuca in a recent review of recovery measures published in the journal Social Work Research (Scheyett, DeLuca, \& Morgan, 2013).

Each member of the expert panel received a draft of the measure and information on the qualitative work that had been conducted to generate the pool of items. Individuals were asked to rate individual items as high, moderate, or low with respect to social recovery, comment on items in terms of fit and clarity, and provide suggestions of other ways to tap into the construct. Feedback of the expert panel was used to further refine the 
draft measure. The second draft follows. Bolded items indicate those that were re-worded for clarity. Unnumbered items were those suggested by the expert panel members.

\section{Community}

1. I have a sense of belonging to a community.

2. I have a sense of safety in community.

3. I have a community that encourages me to grow.

4. I know people who accept me for who I really am.

5. I have real relationships.

6. I have people I can trust.

7. I have relationships that are mutually supportive.

8. I know people who can understand my experiences with mental health challenges.

9. I have a community that recognizes my abilities.

10. I have meaningful things to do.

\section{I have access to the material resources} I need.

I have a community to which I can contribute.

I have a community that values me.

I have opportunities to talk about how mental health challenges have affected my life. I know others who can understand how my mental health challenges connect to my life experiences.

I have relationships that inspire hope.

I have relationships in which I am seen as important.

I have others I can depend on.

I have opportunities to grow.

I am needed by others.

I have relationships in which I am an equal.

I have opportunities to make a contribution.

I am valued for who I am.

I have access to the information I need.

I am able to get my basic needs met.

Self-concept

12. I am an acceptable person.

13. I am not defined by my mental health challenges. 
14. I value my past experiences.

15. I know what I really believe.

16. I know what is true and right for me.

17. I can allow myself to be vulnerable.

18. I am a worthy person.

19. I have something to offer others.

20. I have inner motivation.

21. I am responsible for myself.

I know what I truly value.

I know what it is I really want.

I am acceptable to the people who matter to me.

I am more than my experiences with mental health challenges.

I grew through my experiences with mental health challenges.

I can be effective.

I am a valuable person.

I am capable.

I can both give and receive help.

I can direct myself.

I am responsible for making changes in my life.

I have a purpose in life.

I have a contribution to make.

I have strengths.

\section{Capacities}

22. I have let go of the past.

23 . I can ground to the present moment.

24. I have skills to interact in society.

25 . I can express my inner experiences.

26. I can be my true self.

27. I can pursue my goals.

28 . I can connect with others.

29. I can accept experiences even if they are difficult.

30. I choose which relationships are right for me.

31. I can cope with mental distress.

32. I have hope for the future.

I can make my voice heard.

I can exercise my rights.

I can make sense of my experiences with mental health challenges.

I find meaning in life.

I can express myself.

I can lead a full life. 
I can make my own decisions.

I can be optimistic.

I can make a contribution.

I can set my own goals.

I have abilities to meet goals.

I look forward to the future.

I plan for my future.

Another focus group was held with individuals who identify as having lived experience but who are not involved in academia. The input from the focus group members was further explored in a series of five interviews with individuals with lived experience who are not involved in academia. The focus group and interviews took place in March 2015. Participants were recruited from Folk Time socialization programs in the Portland, Oregon metro area and were compensated with $\$ 20$ stipends. Five participants identified as male and five identified as female. Ages ranged from 20 to 68. Seven participants identified as Caucasian, one as Asian-American, and two as AfricanAmerican. Eight participants stated that they were diagnosed with schizophrenia and two stated that they were diagnosed with depression. The focus group and interview participants further refined the measure through examination of both the content the items were constructed to assess and the feasibility of the resulting measure. Items should address areas of recovery and should be worded in such a way as to be accessible to those who may take the measure. The next draft of the measure follows. Bolded items indicate those that were reworded or added:

\section{Community}

1. I have a sense of belonging to a community. I feel I belong to a community.

2. I have a sense of safety in community.

3. I have a community that encourages me to grow.

4. I know people who accept me for who I really am. 
5. I have real genuine relationships.

6. I have people I can trust.

7. I have relationships that are mutually supportive.

8. I know people who can understand my experiences with mental health challenges.

9. I know people who can understand my challenges.

10. I have a eommunity supportive group that recognizes my abilities.

11. I have meaningful things to do.

12. I have access to the material resources I need.

13. I have a community to which I can contribute.

14. I have a community that values me.

15. I have opportunities to talk about how mental health challenges have affected my life.

16. I know others who can understand how my mental health challenges connect to my life experiences.

17. I have relationships that inspire hope.

18. I have relationships in which I am seen as important.

19. I have others I can depend on.

20. I have opportunities to grow.

21. I am needed by others.

22. I have relationships in which I am an equal.

23. I have opportunities to make a contribution.

24. I am valued for who I am.

25. I have access to the information I need.

26. I am able to get my basic needs met.

27. I have a supportive group that I do enjoyable things with.

Self-concept

28. I am an acceptable person.

29. I am not defined by my mental health challenges.

30 . I value my past experiences.

31 . I know what I really truly believe.

32. I know what is true and right for me.

33. I can allow myself to be vulnerable. I can be vulnerable.

34. I can share myself with others.

35. I am a worthy person.

36. I have something to offer others.

37. I have inner motivation.

38. I am responsible for myself.

39. I know what I truly value.

40. I know what it is I really want.

41. I am acceptable to the people who matter to me.

42. I am more than my experiences with mental health challenges.

43. I grew through my experiences with mental health challenges. 
44. I can be effective.

45. I am a valuable person.

46. I am capable. I am a capable person.

47. I can both give and receive help.

48. I can direct myself. my life.

49. I am responsible for making changes in my life.

50. I have a purpose in life.

51. I can grow.

52. I have a contribution to make.

53. I have strengths.

54. I can enjoy things I do.

Capacities

55. I have let go of the past.

56. I can live in the present instead of being focused on the past.

57. I have skills to interact in society.

58. I can express my inner experiences.

59. I can express my thoughts and emotions.

60. I can be my true self.

61. I can be aware of my true self.

62. I can pursue my goals.

63. I can connect with others.

64. I can accept experiences even if they are difficult.

65. I choose which relationships are right for me.

66 . I can cope with mental distress.

67. I have hope for the future.

68 . I can make my voice heard.-I can voice my opinions.

69. I can express myself.

70. I can exercise my rights.

71. I can exercise my personal freedoms.

72. I ean make sense of my experiences with mental health challenges.

73. I find meaning in life. My life is meaningful.

74. I can lead a full life.

75. I can make my own decisions.

76. I can be optimistic.

77. I can make a contribution.

78. I can set my own goals.

79. I have abilities to meet goals.

80. I look forward to the future.

81. I plan for my future.

82. I have a future. 


\section{I know what I want from my future.}

\section{I think about my future.}

85. I can be present instead of anxious.

\section{I can live in the present.}

\section{I have things I enjoy doing.}

\section{Reading Level}

Items were written at a $6^{\text {th }}$ grade reading level as evaluated by the Flesch-Kincaid Grade Level Test and rated on a 5-point Likert scale. Response options ranged from $1=$ "strongly disagree" to 5 = "strongly agree." A 5-point Likert scale was selected for two reasons. First, Likert scaling provides gradations of responses and offers respondents choices in "equal appearing intervals." Each text label is associated with a number on the continuum. The numbers are equated to text labels that are perceived as having equal distance between each response choice. The intention is that association of labels with numbers will allow respondents to express their true thoughts. Second, an odd number of response categories offers an option of a neutral selection choice and allows the respondent to indicate equal attraction to both sides of the response choices. Should a respondent have a neutral feeling towards an item, the lack of such an option disallows an opportunity for expression and leads to inflated measurement error (Abel, Springer, \& Kamata, 2009).

The next sections will explore the conceptual framework of measurement instruments and reliability and validity concepts. Following the exploration, measures to be used for convergent construct validity will be reviewed. The chapter will conclude with a presentation of the procedures used for working with research participants, managing data, and testing the psychometrics of the Social Recovery Measure. The technical 
information in the following sections was drawn primarily from the work of DeVellis (2012) and Abel, Springer, and Kamata (2009) except where otherwise noted.

\section{Conceptual Framework}

Theory has a central role in the development of measurement instruments. Measurement instruments consisting of items that combine for a composite score may be constructed so as to reveal levels of theoretical variables that are not directly observable. Such measures consist of what may be termed "effect indicators" or items whose values are caused by an underlying construct or latent variable. The latent variable is the actual phenomenon of interest and is most typically a characteristic of an individual who reports the data. Use of a number of items is necessary as the latent variable cannot be directly observed. The classical measurement model assumes that individual items are comparable indicators of the latent variable. The latent variable has two main features. It is latent rather than manifest and it is variable rather than constant. This latent variable is regarded as the cause of items and their values. The score of an item is caused by the strength of the latent variable for an individual at a particular time.

As each item is theoretically caused by the latent variable, the items should correlate with one another. Examining how items correlate with one another allows us to see how highly correlated each item is with the latent variable. The classical measurement model assumes that: (a) the amount of error associated with individual items varies randomly. Item means tend to be unaffected by error given a large number of respondents; (b) one item's error term is not correlated with the error term of another item. Items are linked 
only through the latent variable; and (c) error terms are not correlated with the true score of the latent variable.

\section{Reliability}

Reliability is defined as the internal consistency of an instrument or its ability to measure the same matter each time it is used. A reliable scale yields scores that represent the state of the latent variable being assessed. The score should not change unless there is an actual change in the variable being measured. A perfect scale would reflect a true score and nothing else. In the most formal terms, scale reliability is the proportion of variance in actual scores that is attributable to the true score on the latent variable.

Reliability is conceptually understood as the composite of two unobserved components: the true score and an error score. Each person's observed score $(\mathrm{O})$, consists of a "true" score, T, and a random error score, E. The relationship is depicted in the equation $\mathrm{O}=\mathrm{T}+\mathrm{E}$. Random errors affect a score due to pure chance and reduce both consistency and the usefulness of the scores. The true score can be thought of as the score under perfect conditions or as the average of the observed scores obtained over an infinite number of repeated administrations of the instrument. The true score can also be said to be what the rater actually experiences and the error score can be said to be the gap between the experience and what is observed of the experience.

There are several types of reliability. Each rests on a conceptual foundation of reliability consisting of the proportion of variance in an observed score that can be attributed to the true score of the variable being assessed. Test-retest reliability is consistency over time. An individual should give the same responses at time one as two 
weeks later at time two as long as the latent variable is unchanged. The correlation coefficient between the two sets of scores, or the coefficient of stability, is used to measure this type of reliability. It is generally reported as the correlation of time one scores with time two scores. The parallel-form method also involves two testing situations with the same people but uses different forms of a test that purports to measure the same thing. Two tests are defined as parallel when each respondent has the same true score on both forms of the test and the error variances for the two forms are equal. The reliability coefficient can be defined as the correlation between scores on parallel test forms. The split-halves method involves administering one form of the instrument to a group of subjects. Prior to scoring, the items are divided to create two halves of the test. The correlation between the two halves provides the estimate of reliability.

Internal consistency reliability involves the homogeneity of scale items. The relationships among items should be logically connected to the relationships of items to the latent variable. If the items are strongly related to the latent variable, they will have strong relationships to one another. Internal consistency is evaluated by the extent that items are highly intercorrelated. Each dimension of a measure should consist of a set of items that correlate well with one another. The estimation of internal consistency is commonly calculated using Cronbach's coefficient alpha which provides the proportion of variance in a scale that is attributable to the true score of the latent variable.

Reliability in this study will be measured using the Cronbach's coefficient alpha statistic and the test-retest method. Cronbach's alpha utilizes inter-item correlations to determine if test items are correlated with one another. It is based on the average 
correlations among the items. When respondents perform consistently across items within an instrument, item homogeneity is indicated. If items demonstrate good internal consistency, the Cronbach alpha exceeds 0.70 for a developing instrument and 0.80 for an established instrument. Test-retest reliability was conducted using a subsample of 30 individuals who were administered the test once and then again two weeks later. A testretest statistic of .75 was considered acceptable (Bowling, 2009; Pallant, 2010).

\section{Standard Error of Measurement (SEM)}

Approaches to reliability are designed to assess the error in the measure. The standard error of measurement (SEM) describes the expected variation of the true scores. It is a method of examining reliability by directly estimating the amount of error in the instrument. Each subject's test score has a standard deviation. SEM is a result of the average of these individual standard deviations. It estimates the standard deviation of error. As an error index, the SEM can be used to determine what change in scores may be due to error.

SEM provides an interval estimate of how far the true score may lie from an observed score. It can be used to construct a confidence interval. A scale with low SEM will have a narrow confidence level and good measurement error characteristics. The smaller the SEM, the less measurement error and the greater instrument reliability. A SEM that is 5\% or less of the range of possible scores is recommended. A standard error of measurement was computed for each of the final domains. 


\section{Item Pool}

As reviewed previously, all items of a scale should reflect the underlying latent variable. Multiple items will make for a more reliable test than individual items. Some redundancy is necessary for an effective instrument. Redundancy is actually the foundation of internal-consistency reliability. Early in the scale development process, it is better to have more item variants than later proves necessary, than too few items with which to begin. Item generation should be focused on revealing the phenomenon of focus in different ways. The use of multiple and apparently redundant items can express relevant ideas and result in capturing the construct.

Useful redundancy is concerned with the underlying variable and is not created through grammar or vocabulary changes. It is best to explore the nuances of the meaning of a construct by examining different ways of expressing the varied aspects. Varied representation of a common theme can enhance both content validity and internal consistency. As long as items are not literally identical, repetition of item content can be a means of viewing an issue from all angles. Subsequent psychometric analyses will separate the good items from the bad.

Items that perform well are clear and unambiguous, brief, written at an eighth grade or lower reading level, and express only one idea. Positively worded items are those that represent the presence of the construct. Negatively worded items are defined as those that indicate low levels or the absence of the construct. Negatively worded items are sometimes included with the intention to avoid acquiescence, affirmation, or agreement bias or the tendency of respondents to answer items less on the basis of content than on a 
pattern of responding. The response bias refers to a respondent's tendency to agree with items regardless of their content. However, inclusion of both positively and negatively worded items can be confusing for the respondent and may not lead to improvement in the instrument. In the interest of clarity and ease of instrument use for the respondents, the Social Recovery Measure will feature only positively worded items. However, items from the different meta-themes or proposed domains were randomly distributed on the version of the instrument administered to respondents.

\section{Validity}

Validity is associated with the underlying construct the instrument is intended to measure. Therefore, it is related to theoretical issues. An instrument has utility to the extent that it is reflective of the underlying theoretical concept. Scores on an instrument should be representative of the construct in question. Validity concerns whether or not the latent variable is the underlying cause of the covariance of items. DeVellis (2012) stresses that validity of a scale is not firmly established during development, but is a cumulative, ongoing process. There are several types of validity. Content validity is achieved when all items are clearly related to the construct. The set of items should reflect a domain of content. Construct validity is concerned with the theoretical relationship of a variable to other variables and is a measure of how well the instrument fits the ideas of a construct. It is evaluated by discovering how a scale that purports to measure a construct displays a similar relationship to other measures of the construct. Factorial validity is a form of construct validity. It is established through a factor analysis or procedure for analyzing the interrelationships among a set of variables and for 
explaining the interrelationships in terms of a reduced number of variables or factors. Concurrent validity is evaluated by how highly positively correlated a measure is with another known measure of a similar type and how negatively correlated a measure is with an opposite construct. Predictive validity status is achieved when a measure predicts a practical result or important outcome (Campbell-Orde, Chamberlin, Carpenter, \& Leff, 2005).

\section{Content Validity}

Content validity is concerned with how well the content of items reflects the target construct. Content validity depends on both the original construction of items and the expertise and judgment of individuals selected to review them. Expert reviewers should be selected based on knowledge of the substantive area and/or capacity to understand the psychometric task at hand. Recommendations for expert panelists include a balance of involvement of those who have lived experience of the substantive area and those who have studied it. The goal of expert review is to cross-check, revise, and potentially eliminate items before collecting sample data.

\section{Factorial Validity}

Factorial validity is a form of construct validity that is established through a factor analysis. The two types of factorial validity are exploratory and confirmatory factorial analysis. A factor analysis is purely exploratory in the absence of a hypothesis about the number or nature of the factors or latent variables measured by the test. A factor analysis is confirmatory in cases in which there is a strong theory and/or empirical base and when the number of factors or domains has been established. A confirmatory factor analysis 
(CFA) was used in this study given the extensive work that has gone into establishing measurable dimensions of key recovery processes. A CFA was conducted to establish the degree to which specific items on the Social Recovery Measure are correlated with the three domains derived from the focus group data or with the five domains of the CHIME framework. Factorial validity indicates only that a test is properly classified, not necessarily that it is good or useful for any purpose.

\section{Construct Validity}

Construct validity is known to be the most elusive of the validity types and is closely tied to theoretical considerations. It concerns the theoretical relationships of a variable to other variables. It requires integration of multiple lines of evidence. Construct validity is typically tied to two subtypes: convergent and discriminant. Convergent construct validity tests whether a construct correlates with a theoretically relevant variable with which is expected to correlate. A new scale should correlate significantly with established instruments that measure related constructs and should strongly negatively correlate with established instruments that measure concepts that are the opposite of the concept being measured in the new scale. Use of established measures of relevant constructs can provide support for validity when developing a measure. The performance of the measure compared to other measures can serve as the recommended validation step for measure development.

\section{Determination of Convergent Validity}

Convergent validity was determined with the Recovery Assessment Scale (RAS; Giffort, Schmook, Vollendorf, \& Gervain, 1999), the Questionnaire about the Process of 
Recovery (QPR; Neil, Kilbride, Pitt, Nothard, Welford, Sellwood, \& Morrison, 2009) and the Beck Hopelessness Scale (BHS; Beck, Weissman, Lester, \& Trexler, 1974). It was hypothesized that scores on the RAS, particularly the "Personal Confidence and Hope," "Reliance on Others," and the "Goal and Success Orientation" subscales, scores on the QPR would be positively correlated, and scores on the BHS would be negatively correlated with the Social Recovery Measure. Measures were selected based on similarity and difference respectively in terms of the construct of social recovery as well as on their established psychometric properties.

\section{Measures}

The following three instruments were administered to participants in this study in order to establish convergent construct validity. A brief description and available psychometric properties of each instrument is presented.

The Recovery Assessment Scale (RAS). The Recovery Assessment Scale (RAS; see Appendix B) was developed in the United States to measure personal recovery in adults. The RAS is based on a process model of recovery as opposed to a stage model and has a particular focus on hope and self-determination. The measure was developed through narrative analysis of four consumer recovery stories and was originally a 39-item scale. An independent group of twelve consumers subsequently reviewed the scale items and revisions led to the current 41 -item instrument. The subscales of the RAS measure five domains: personal confidence and hope, willingness to ask for help, goal and success orientation, reliance on others, and not being dominated by symptoms. Items are scored on a 5-point Likert scale that ranges from $1=$ "strongly disagree" to $5=$ "strongly agree." 
A higher score indicates a stronger state of recovery. A confirmatory factor analysis validated the five factor structure. The alphas for the five factors ranged from .74 to .87 : personal confidence and hope (alpha $=.87)$; willingness to ask for help (alpha $=.84)$; goal and success orientation $($ alpha $=.82)$; reliance on others $($ alpha $=.74)$; no domination by symptoms (alpha $=.74)$. Internal consistency has been evaluated as a Cronbach's alpha $=.93$. Test-Retest Reliability (14 day interval) has been evaluated as a Pearson's Product Moment Correlation $r=.88$ (Campbell-Orde, Chamberlin, Carpenter, \& Leff, 2005)

The RAS appears to be the most widely published and studied measure of recovery currently available. As of August 2012, a total of 222 articles published around the world were found to utilize the RAS although only 77 of the articles included psychometric data on the instrument (Salzer \& Brusilovskiy, 2014). In a systemic review of measures of personal recovery, the RAS was evaluated as demonstrating an adequate demonstration of the property for content and construct validity and for internal consistency $(.89, .93$ and range .73-91) and test-retest reliability (range .81, .88, and range .39-.83). No information was available to evaluate criterion validity, responsiveness, time to complete, reading age, or feasibility (Shanks, Williams, Leamy, Bird, Boutillier, \& Slade, 2014). The RAS is not copyrighted, can be downloaded free of charge, and may be used freely. I hypothesized that the Social Recovery Measure will be most strongly correlated with the RAS "Personal Confidence and Hope," "Goal and Success Orientation," and "Reliance on Others" subscales. The Social Recovery Measure will be less focused than the RAS on items reflected on the "Willingness to ask for Help" and "Not Dominated by 
Symptoms" subscales. Compared to the RAS, the Social Recovery Measure focused less on seeking assistance and managing symptoms and focus more on building mutually supportive relationships, constructing a positive identity, and pursuing personal goals.

The Questionnaire about the Process of Recovery (QPR). The Questionnaire about the Process of Recovery (QPR; see Appendix B) was developed in the United Kingdom as a measure of recovery specific to psychosis. The 22 -item measure was designed to be used as a tool for setting and measuring goals. Two service user researchers were part of the study team which worked with a steering committee of 10 service users. The QPR was grounded in a theme of recovery as a process and was developed from interviews with 126 individuals with lived experience of psychosis. Of the 126 participants initially interviewed, $111(88 \%)$ engaged in the next phase of measure generation. The subscales of the QPR measure two domains: intrapersonal (17 items) relating to tasks an individual is responsible for carrying out in order to rebuild a life and interpersonal (5 items) relating to external processes and relationships with others. Items are scored on a 5-point Likert scale that ranges from $0=$ "disagree strongly" to $4=$ "agree strongly." Higher scores are indicative of recovery. Cronbach's alpha coefficients (intrapersonal subscale 1 $=.94$ and interpersonal subscale $2=.77$ ) indicated good internal consistency. Test-retest reliability compared the scores of 43 participants from one week to the scores two weeks later. Results (intrapersonal subscale $r=.874, p=.001$; interpersonal subscale $r=.769, p$ $=.001)$ indicated good test-retest reliability. The developers of the measure discussed that the finding of two subscales, intrapersonal and interpersonal, was consistent with previous research that recovery is supported by both internal factors such as hope, 
empowerment, confidence, and purpose, and external factors such as connectedness and caring and support from others (Neil et al., 2009).

A systematic review of measures of personal recovery evaluated measures based on fit with the CHIME recovery processes and for demonstration of psychometric properties. The QPR was the only measure of thirteen identified to have all items match to the CHIME conceptual framework. It was evaluated as demonstrating an adequate demonstration of the property for content and construct validity and for internal consistency (subscale $1=.94$; subscale $2=.77$ ) and test-retest reliability (intra subscale $r$ $=.874$; inter subscale $r=.769$ ). No information was available for criterion validity, responsiveness, time to complete, reading age, or feasibility (Shanks et al., 2014). Another evaluation of the psychometric properties of the QPR found that internal consistency could be improved by removing seven of the 22 items. Five of the seven items targeted for removal constitute the entire interpersonal subscale. The resulting QPR would fit a one factor model that tested for an intrapersonal domain (Law, Neil, Dunn, \& Morrison, 2014).

I hypothesized that the Social Recovery Measure would be most closely correlated with the intrapersonal subscale of the existing QPR. The items of the intrapersonal subscale focus on positive self-identity, meaning, empowerment, sense of connectedness, self-direction, hope, and relationship and community development. However, the intrapersonal subscale also includes items focused on symptom issues which distinguishes the subscale from the SRM. The items of the interpersonal subscale focus on seeking care and attitudes regarding engagement in advocacy. As the Social Recovery 
Measure does not address seeking care or engaging in advocacy, it was not expected to correlate strongly with the particular QPR items.

The Beck Hopelessness Scale (BHS). The Beck Hopelessness Scale (BHS, see Appendix B) is the most widely used measure of hopelessness in the psychiatric, medical, and general populations (Kao, Liu, \& Lu, 2012). Further, it has been used in the testing of construct validity of the QPR and other measures of recovery (Law, Neil, Dunn, \& Morrison, 2014). The BHS is a 20-item self-report measure designed by clinicians to measure negative expectancies. As originally constructed and currently available, the measure covers three aspects of hopelessness: feelings of the future, loss of motivation, and expectations. Statements are rated by participants as true or false for their attitudes over the last week. The possible range of scores is from 0 to 20 .

The psychometric characteristics of the BHS were first examined with a sample of 294 patients who had attempted suicide. The BHS was found to be reliable (.93) with itemtotal correlation coefficients ranging from .39-.76. The three factors emerged from a principal components factor analysis with varimax rotation (Beck, Weissman, Lester, \& Trexler, 1974). The measure was subsequently subjected to an item analysis with general and forensic psychiatric populations and initial psychometric findings were replicated (Durham, 1982). An evaluation of the scale using item-response theory with two separate clinical samples found the scale to be unidimensional. In the one-factor model, all items demonstrated an acceptably strong relationship to the latent variable of hopelessness. Coefficients were over 90 (Young, Halper, Clark, Scheftner, \& Fawcett, 1992). In a general clinical population, Dyce (1996) found the measure to be internally 
consistent $(r=.92)$ and the three-factor solution supported using principal-component analysis with both varimax and oblique rotations. Steed (2001), employing a non-clinical sample, found the measure to be internally consistent $(r=.88)$ with item-total correlations ranging from .29 to .66 . Steed proposed a two-factor solution after carrying out varimax and oblique rotations and conducting confirmatory factor analyses. The psychometric properties of the measure were further evaluated on an outpatient sample of individuals diagnosed with schizophrenia. A factor analysis with a principal components analysis after oblimin rotation yielded two factors: negative expectation for the future and loss of motivation. The measure was found to be internally consistent $(r=.85)$ with moderate to moderately high item-total correlations. A test-retest at two weeks reliability procedure demonstrated highly significant relationships for all rating scores $(r=.80, N=$ 30, $p<.001 ;$ Kao, Liu, \& Lu, 2012).

Procedure

The Portland State University Institutional Review Board Office of Research Integrity granted approval to the study (Appendix A) The Informed Consent form was written at an eighth-grade reading level. A Qualtrics electronic survey was compiled and distributed. The survey included demographic questions, the draft Social Recovery Measure, and the RAS, QPR, and BHS (Appendix B).

\section{Participants}

A nonprobability, purposive sample of 228 individuals with lived experience of mental health challenges took the survey between April 2015 and June 2015. Data were gathered onsite and online from: Folktime; Comfort Zone; NorthStar; Lifeworks NW; 
Columbia County Mental Health; Recovery Outreach Recovery Center in the greater Portland, Oregon metro area. Data were gathered online from consumer operated programs: VOCAL Virginia; New York Hearing Voices; Seattle Hearing Voices; Western Massachusetts Recovery Learning Centers; Montana’s Peer Network; Georgia Mental Health Consumer Network, Inc; the Pennsylvania Mental Health Consumer's Association; and Project Return Peer Support Network in California.

\section{Risk and Informed Consent}

It was anticipated that participation in the study would present no more than minimal risk to the participants. Research participants may have experienced some psychological discomfort as completing the measures may have brought up some emotions. All participants were receiving peer support or mental health services and I also offered my assistance in processing emotional responses.

Participation was entirely voluntary. Participants were not asked to sign informed consent forms and were informed that they did not have to participate in the study and would experience no negative effects from declining. The researcher's name was given as the contact person in the event that a participant or gatekeeper had questions or concerns related to the study. Participants were also given the contact information for the PSU IRB in the event of perceptions of rights violations or harms sustained from study participation. Participants who completed the measurement packets were offered the opportunity to be entered into a drawing for one of two $\$ 100$ Visa gift cards. The subsample of participants who engaged in a test-retest procedure were offered the opportunity to enter a second drawing for a $\$ 50$ gift card. Participants who chose to enter 
the drawings were sent to a non-linked Qualtrics page. They were asked for their contact information but could chose to use an alias as long as they could be contacted in the event they won the drawing.

\section{Data Collection and Entry}

The electronic data will be kept on a secure drive of Portland State University. Confidentiality was maintained by assigning each respondent a non-identifiable number associated with the corresponding electronic packet. The researcher imported data from the Qualtrics files to SPSS 21 and then from SPSS 21 to SPSS Analysis of Moment Software (AMOS). AMOS is used for structural equation modeling (SEM).

\section{Missing Data}

Missing data are a result of intentional or unintentional unanswered items. As missing data reduce the sample size and degrees of freedom when conducting statistical analysis, the power of a study to detect statistically significant relationships or differences in the data can be diminished. The prevalence of unanswered items is determined after data collection and entry. Screening for missing data involves (a) determining how much data are missing and identifying whether there is a random or systematic pattern to the missing data; (b) determining why data are missing; and (c) deciding on the best approach to managing missing data (Duffy, 2006). Random error refers to error that lacks a pattern across respondent groups, while systematic error refers to errors with a statistically observable pattern. Systematic error affects the generalizability of study findings (Munro, 2005). Several approaches exist to adjust for missing data. The most appropriate approach is based on the degree of randomness of missing response items. 
Generally, if less than $5 \%$ of the data are absent without a definite pattern, most procedures for missing data may be used (Munro, 2005; Tabachnick \& Fidell, 2007). In the case of systematic error in the data, the number of procedures is reduced. Missing Value Analysis (MVA) is a procedure available in SPSS for addressing missing data. MVA involves three functions: (a) description of the pattern of missing data; (b) estimation of means, standard deviation, correlations and co-variances for several missing value methods (listwise, pairwise, regression and expectation-maximization (EM); and (c) filling in missing data (Pallant, 2010). Neither listwise nor pairwise missing value methods require the third step of filling missing data since they involve removing cases in their entirety. Regression and EM are processes used to impute missing data. Both involve the imputation of one complete data set. Multiple Imputation yields more than one complete data set and the standard errors are estimated in order to establish parameters (Little \& Rubin, 1987). Generation of complete data sets reduces the chance of error related to replacement of missing values. If data are found to be missing in a non-random manner, neither regression nor EM may be used. Many recommend repeating statistical analysis with and without missing data replacement as a check against distortion (Duffy, 2006). As the amount and type of missing data cannot be predicted, the appropriate response to the issue is to be made after assessing the amount and type of missing data. Only six of 228 participants did not provide demographic data and no other data were missing. No missing data procedures were required. 


\section{Skewness and Kurtosis}

Normal distribution of data is one of the major assumptions of structural equation modeling. Skewness and kurtosis provide information on the distribution of scores on a continuous variable and are measures of departures from normality. Departures from normality can affect tests and confidence levels. The shape of a data distribution is relevant to the issue of the assumptions and robustness of analysis procedures. Skewness provides an indication of the symmetry of the distribution and affects the test of means. Kurtosis provides information about the peakedness of the distribution and impacts tests of significance, standard errors of parameters, variance, and covariance. A perfectly normal distribution would have skewness and kurtosis values of 0 (DeCarlo, 1997; Pallant, 2010). Skewness and kurtosis was calculated for each instrument item. While there are differences of opinion regarding acceptable values, an absolute kurtosis value greater than 10 and an absolute skewness value greater than 3 were to be considered as indicative of departure from normality (Kline, 2011).

\section{Item Evaluation}

The performance of each item requires evaluation so that the most appropriate ones will constitute the measure. An ideal item is highly correlated with the latent variable. Relationships to the latent variable can be discerned through correlations among items. The higher the correlations among items, the higher the individual item reliability. The more reliable the individual items are, the more reliable the measure will be as they will share a common latent variable. High intercorrelation of items is highly desirable. In order to realize high intercorrelation of items, each item should correlate substantially 
with the collection of remaining items. This is examined by computing item-scale correlation. The corrected item-scale correlation procedure correlates the item being evaluated with all the scale items excluding itself. An item with a high value for this correlation is desirable. A desirable item will also have relatively high variance and a mean close to the center of the range of possible scores. An item that does not vary cannot covary, so an item with low variance or a skewed mean will have low correlation with other items. Inspection of variances and means can serve as check to item correlations.

\section{Coefficient Alpha}

Coefficient alpha is an indicator of a scale's reliability. Conceptually, it is an estimate of how much the covariances between items account for the variance of the total test scores. It is indicative of the proportion of variance in the scale score that is attributable to the true score of the latent variable. A non-central mean, poor variability, low itemscale correlation, and weak inter-item correlations will reduce alpha. A scale's alpha is influenced by the extent of covariation among items and the number of items on the scale. Inclusion of more items with good item-scale correlations will increase alpha and removal of such items will lower alpha. Alpha can theoretically range from 0.0 to 1.0. An alpha between .7 and .8 is generally considered respectable and an alpha between .8 and .9 is generally considered very good. For this study, alphas of .7 and above were sought (Nunnally, 1978). While longer scales tend to be more reliable, shorter scales place less burden on respondents. Therefore, attention was placed on an acceptable tradeoff between burden and reliability. 


\section{Factor Analysis}

In factor analysis, observed variables are modeled as a function of underlying unobserved variables. Item responses on a scale are observed variables and are modeled as a function of latent variables. Observed item responses are predicted by the latent variable. Factor analysis allows for the determination of the number of factors underlying a set of items and provides insight into the nature of the latent variables. Factor analysis can also provide a means of explaining variation among the original variables. It defines the substantive content or meaning of the factors that account for the variation among items. It also assists with identification of item performance. Individual items that do not fit into any of the factorial-derived categories of items or that fit into more than one of the categories can be identified and considered for removal.

The first task with factor analysis is to determine the number of categories sufficient for capturing the majority of information contained in the original set of items. The analysis assesses how much of the association among individual items can be explained by a single concept. If one concept or category cannot adequately account for the covariation among items, the analysis identifies one or more additional categories until the amount of unaccounted for covariation is acceptably small. Conducting a factor analysis can result in examining a large set of items or variables and determining a smaller set of factors that capture or condense the original information.

An eigenvalue represents the amount of information captured by a factor. For example, the total amount of information in a set of items is equal to the number of items. Given 25 items, there would be 25 units of information. Each factor's eigenvalue 
corresponds with some portion of those units. An eigenvalue of 1.0 corresponds to $1 / \mathrm{k}$ of the total variance among a set of items. A factor with eigenvalue 1.0 contains the same proportion of total information as a typical single item. According to the eigenvalue rule, factors with eigenvalues less than 1.0 should not be retained. The scree test is based on a plot of the relative, as opposed to the absolute, eigenvalues.

\section{Rotating Factors}

Factor rotation is a necessary step in interpretation. It acts to identify clusters of variables that can be characterized in terms of a single latent variable. It performs this task by selecting vantage points from which to describe items and their relationships. In the patterns of intercorrelation among a set of items, the stronger the association of items, the closer to one another they could be said to be. If factors or the underlying latent variables are thought to be strongly correlated, an oblique rotation should be specified.

\section{Confirmatory Factor Analysis}

Confirmatory factor analysis (CFA) is a factor analysis method that that is applied to a set of items to confirm a particular pattern of relationship that is predicted by theory or previous analysis. The method is based on structural equation modeling (SEM). SEM is a model that contains structural relationships between variables and latent factors. A CFA is special case of SEM. SEM-based approaches provide a statistical criterion for evaluating how well the real data fit the specified model. The analyst specifies the anticipated relationship among variables and the computer program performs calculations allowing the analysis to determine if the model can be reconciled with the data. Structural equation modeling produces plausible solutions. Judgment comes into play in making the 
best choice among available solutions. The initial step of a CFA is to specify the number of factors or the number of subscales. The next step is to specify which items are commonly predicted by the same latent variable or, in other words, which items load on which factor or subscale. The three-factor rule requires a minimum of three items per factor (Nunnally, 1978).

\section{Model Fit}

If the model fits the data, there is evidence to confirm the theoretical factor structure. If the model does not fit the data well, responses to the instrument are not consistent with the theoretical factor structure of scale items and revision of the instrument is indicated. Goodness of fit estimates are used to determine how well the hypothesized measurement model fits the observed correlation. There are a number of model fit indices and statistics.

A chi-square goodness of fit statistic tests a null hypothesis that the data covariance and the reproduced covariance are the same. Rejecting the null hypothesis indicates that there is enough evidence that the model does not fit the data. The conclusion would be that there is a poor fit of the model to the data (Nunnally, 1978). As the null hypothesis is that the postulated model holds in the population, in contrast to traditional statistical procedures, the researcher in this case hopes not to reject the null hypothesis. The chisquare is sensitive to sample size. With small samples, there may not be enough power to detect differences between models. The relative chi-square is the chi-square divided by degrees of freedom and is abbreviated as CMIN/DF. It is an attempt to make the test less dependent on sample size. The smaller the chi-square, the better the fit of the model. A CMIN/DF ratio below 3 is generally considered acceptable. The comparative fit index 
(CFI) is related to the difference between the chi-square statistic and its degrees of freedom for the proposed model and for the null model. In the null model, all parameters are constrained to be zero and are analogous to the observed covariance. CFI measures the improvement of fit by the proposed model. The range of the index is restricted to between 0 and 1 with higher values indicating better fit. The standard is that 0.90 is an acceptable fit and above 0.95 is an excellent fit. The Tucker Lewis Index, also known as the Non-Normed Fit Index, evaluates the ratio of the chi-square statistic to its degrees of freedom in the proposed model relative to the same quantity of the null model. A higher value indicates more improvement from the null model. The range is restricted from 0 to 1. A value of 0.90 is considered acceptable and a value above 0.95 is considered an excellent fit. The Root Mean Square Error of Approximation (RMSEA) is based on the degree of noncentrality of the chi-square statistic. The noncentrality quantity indicates the degree of deviation from the null hypothesis. In this context, the null hypothesis states that the data covariance and the reproduced covariance are the same. A perfectly fit model will have a noncentrality parameter of the chi-square statistic of 0 and the RMSEA will also be zero. If the model is not perfect, the noncentrality parameter will have a positive value and a non-zero positive RMSEA. Smaller values indicate a better fit. A good fit will obtain a 0.50 or smaller RMSEA and an acceptable fit is 0.80 or lower. A Standardized Root Mean Square Residual (SRMR) is a measure of discrepancy between the data covariance and the reproduced covariance based on estimated parameter values. A good fit for SRMR is 0.10 or smaller (Hu \& Bentler, 1999). 
The majority of goodness of fit estimates are conducted using the chi-square statistic. Using additional model fit indices complements the chi-square statistic and enhances the integrity of a fit model. The combinational strategy results in lower sums of Type I and Type II error rates and so are preferred criteria for model evaluation. There is a trade-off between Type I and Type II error rates when $\mathrm{N} \leq 250$ as in this study. When sample size is small, most combinational rules have a tendency to over-reject true-population models. RMSEA is one fit index that is particularly known to over-reject true-population models at small sample size (Hu \& Bentler, 1999). This study used the relative chi-square statistic (CMIN/DF), the root mean square error of approximation (RMSEA), the comparative fit index (CFI), and the standardized root mean square residual (SRMR).

Scale: Burden, Length, and Item Sequencing

In general, shorter scales are preferable to longer ones given the burden on participants who complete instruments and providers and researchers who administer them. However, if too much is sacrificed for brevity, the psychometrics of the instrument will be weakened. Shrinking an item pool can leave out important content and lead to a scale that is too simplistic. Inadequate reliability is another risk of inclusion of too few items. One of the first steps in examining psychometric evidence is to estimate the consistency of scale responses. Interpreting coefficient alpha and alpha-of-item-deleted statistics can guide retention of strong items and identification of weak ones for possible deletion. The ideal length of a scale can be determined by the anticipated burden of using it, the complexity of the construct, and the set standards for psychometric strengths. There are three scale development goals that need to be balanced: strengthening internal 
consistency by removal of any weak items; lowering instrument burden by reducing scale length; and easing scale scoring. Factor structure and internal consistency are best tested by randomizing the placement of items on an instrument. Random placement enhances confidence that patterns found are due to a shared construct rather than by close placement on an instrument. Responses should cluster not by response patterns but due to reflection of the common latent construct.

Outcome of Dissertation

The study was designed to produce an instrument by the name of the Social Recovery Measure (SRM). The instrument was grounded in disability and mad theories and is specific to the social aspects of recovery. Development was guided by those with lived experience of mental illness who are in recovery and by key recovery processes as identified in a systematic review of the recovery literature. The process of instrument development followed the systematic approach recommended by experts in the measurement field. The next chapters present the findings of the study and discuss recommendations for future research and implications. 


\section{Chapter 4}

\section{RESULTS}

This chapter will present the results of the measurement development and validation study. Basic demographic information on the study participants will be presented first. Next, the exploratory and confirmatory factor analyses will be presented. The final version of the Social Recovery Measure (SRM) will be introduced as the intended product of the dissertation. Next, results of reliability analyses will be reviewed. Lastly, convergent construct validity will be reviewed in terms of correlations with the Recovery Assessment Scale (RAS), The Questionnaire about the Process of Recovery Questionnaire (QPR), the Beck's Hopelessness Scale (BHS), and with specific demographic indicators.

\section{Participant Characteristics}

The total sample consisted of 228 participants. Of the 228 participants, 123 (54\%) participated on-line and $105(46 \%)$ participated in person. As six individuals completed the measures but did not respond to demographic items, the following information is on 222 individuals. As can be seen in Table 2, 155 (70\%) participants lived in Oregon and $67(30 \%)$ were drawn from consumer operated programs in California, Georgia, Illinois, Massachusetts, Montana, New York, Pennsylvania, Virginia, Washington State, and Wisconsin. Participants ranged from age 18 to over 65 years of age. Young adults between the ages of $18-34$ were $18 \%$ of the participants, $48 \%$ were between the ages of $35-54$, and $34 \%$ were 55 or older. The majority of participants were female (59\%). Males were $36 \%$ of the sample and Transgender/Two-Spirit/Gender queer individuals were 5\% 
of the sample. The majority of participants were Caucasian (77\%) and heterosexual (77\%). Most participants were single (47\%); $37 \%$ were in a relationship, a domestic partnership, or marriage; $13 \%$ were separated or divorced; and $3 \%$ were widowed. The majority of participants lived in urban settings (46\%). Suburban dwellers were $25 \%$ of the participants. Those living in small towns were $23 \%$, and those living in rural or frontier settings were $5 \%$.

Table 2. Demographic Characteristics: Residency, Age, Gender, Race/Ethnicity, Sexual Orientation, Relationship Status, Living Situation

\begin{tabular}{|l|l|l|}
\hline Characteristics & Frequency & Percentage \\
\hline State of Residency & 155 & $70 \%$ \\
Oregon & 67 & $30 \%$ \\
Out of State & 9 & \\
\hline Age & 31 & $4 \%$ \\
$18-24$ & 48 & $14 \%$ \\
$25-34$ & 59 & $22 \%$ \\
$35-44$ & 60 & $26 \%$ \\
$45-54$ & 15 & $27 \%$ \\
$55-64$ & & $7 \%$ \\
$65+$ & 81 & \\
\hline Gender & 130 & $36 \%$ \\
Male & 11 & $59 \%$ \\
Female & & $5 \%$ \\
Transgender/Gender Queer & 171 & \\
\hline Race/Ethnicity & 14 & $77 \%$ \\
White & 8 & $6 \%$ \\
African American & 3 & $4 \%$ \\
Asian/Pacific Islander & 5 & $1 \%$ \\
First Nation/Alaskan Native & 21 & $2 \%$ \\
Latino & 171 & $10 \%$ \\
Mixed Race & 24 & $77 \%$ \\
\hline Sexual Orientation & 2 & $15 \%$ \\
Straight/Heterosexual & 15 & $1 \%$ \\
LGBTQP & & $7 \%$ \\
Asexual & & \\
Prefer not to answer & & \\
\hline
\end{tabular}




\begin{tabular}{|l|l|l|}
\hline Characteristics & Frequency & Percentage \\
\hline Relationship Status & & \\
Single & 104 & $47 \%$ \\
In a relationship & 44 & $20 \%$ \\
Married/Domestic Partner & 38 & $17 \%$ \\
Separated or Divorced & 29 & $13 \%$ \\
Widowed & 6 & $3 \%$ \\
Prefer not to answer & 1 & $.5 \%$ \\
\hline Living Situation & & \\
Urban & 103 & $46 \%$ \\
Small town & 51 & $23 \%$ \\
Suburb & 56 & $25 \%$ \\
Rural/Frontier & 12 & $5 \%$ \\
\hline
\end{tabular}

In terms of education, a small minority had less than a high school education (6\%), $19 \%$ had completed high school, earned a GED, or attended trade school, 25\% attended college, $30 \%$ had earned an Associates or Bachelor's degree, and 20\% held a graduate degree. Individuals receiving disability payments were $38 \%$ of the sample. The rest of the sample participants were working full time (22\%), working part time (17\%), temporarily unemployed (10\%), retired (7\%), or students (6\%). The majority of study participants were receiving both traditional community mental health and peer support services $(65 \%)$ which is reflective of recruitment strategies in this study. For the rest of the sample, 15\% received only peer services or supports, $14 \%$ received traditional community mental health treatment, $3 \%$ received private mental health care, $1 \%$ were in primary care only, and $1 \%$ were mental health providers who were not receiving services. The majority of study participants carried a primary diagnosis of Schizophrenia/Schizoaffective/ Psychotic Disorder (24\%) or Bipolar Disorder (24\%). Depression was the primary diagnosis for $21 \%$ of participants and co-occurring depression and anxiety was carried by $10 \%$. PTSD was the diagnosis of $7 \%$ of the sample, an anxiety disorder was carried by 
$5 \%$, Dissociative Identity Disorder by 5\%, Substance Use Disorder by $1 \%$, and Autism Spectrum or brain injury by $1 \%$. A small percentage (2\%) did not identify a mental health diagnosis but chose to identify as in recovery from a mental health challenge or madness (see Table 3).

Table 3. Demographic Characteristics: Education, Employment, Services Type, Diagnosis

\begin{tabular}{|l|l|l|}
\hline Characteristics & Frequency & Percentage \\
\hline Education & & \\
Less than high school & 13 & $6 \%$ \\
High School/GED & 36 & $16 \%$ \\
Trade School & 6 & $3 \%$ \\
Attended college & 57 & $25 \%$ \\
AA/AS & 13 & $6 \%$ \\
BA/BS & 53 & $24 \%$ \\
Graduate Degree & 44 & $20 \%$ \\
\hline Employment & & \\
Working full time & 50 & $22 \%$ \\
Working part time & 37 & $17 \%$ \\
Student & 14 & $6 \%$ \\
Temporarily unemployed & 21 & $10 \%$ \\
On disability & 55 & $38 \%$ \\
Retired & 15 & $7 \%$ \\
\hline Type of Services & \\
Traditional community & 31 & $14 \%$ \\
Peer services or supports & 34 & $15 \%$ \\
Both traditional and peer & 145 & $65 \%$ \\
Primary care & 3 & $1 \%$ \\
Private mental health & 7 & $3 \%$ \\
Mental health provider & 2 & $1 \%$ \\
\hline
\end{tabular}




\begin{tabular}{|l|l|l|}
\hline Characteristics & Frequency & Percentage \\
\hline Primary Diagnosis & & \\
Schizophrenia/Schizoaffective/Psychosis & 54 & $24 \%$ \\
Bipolar disorder & 54 & $24 \%$ \\
Depression & 47 & $21 \%$ \\
Depression and anxiety & 21 & $10 \%$ \\
PTSD & 15 & $7 \%$ \\
Anxiety disorder & 10 & $5 \%$ \\
Dissociative identity disorder & 10 & $5 \%$ \\
Substance use disorder & 3 & $1 \%$ \\
Autism spectrum & 2 & $.5 \%$ \\
Brain injury & 1 & $.5 \%$ \\
In mental health recovery/madness & 5 & $2 \%$ \\
\hline
\end{tabular}

Skewness and Kurtosis

Normal distribution of data is one of the major assumptions of structural equation modeling. Assessment of skewness and kurtosis is one way to assess normality of a sample distribution. Skewness affects the test of means with a positive skew indicating that most of the scores are below the mean and a negative skew indicating the opposite. Kurtosis impacts tests of variance and covariance. A positive kurtosis indicates heavier tails and a higher peak and negative kurtosis indicates the opposite relative to a normal distribution. As with many statistical analyses, there are differing opinions as to the appropriate threshold of non-normality for both skewness and kurtosis. An absolute skewness value greater than 3 and an absolute kurtosis value greater than 10 were considered indicative of departure from normality for this project following conservative guidelines as set by Kline (2011). For the draft measure as a whole, the skew statistic was -.741 (SE .161) and the kurtosis statistic was .864 (SE .321). Skewness and kurtosis were calculated for each item on the draft measure. The largest absolute skew was 1.549 and the largest absolute kurtosis was 3.550 (see Appendix C). As skewness and kurtosis 
indices were well within an acceptable range, no transformations were conducted. See Table 4 for information on how data were distributed.

Table 4. Draft SRM Distribution Data

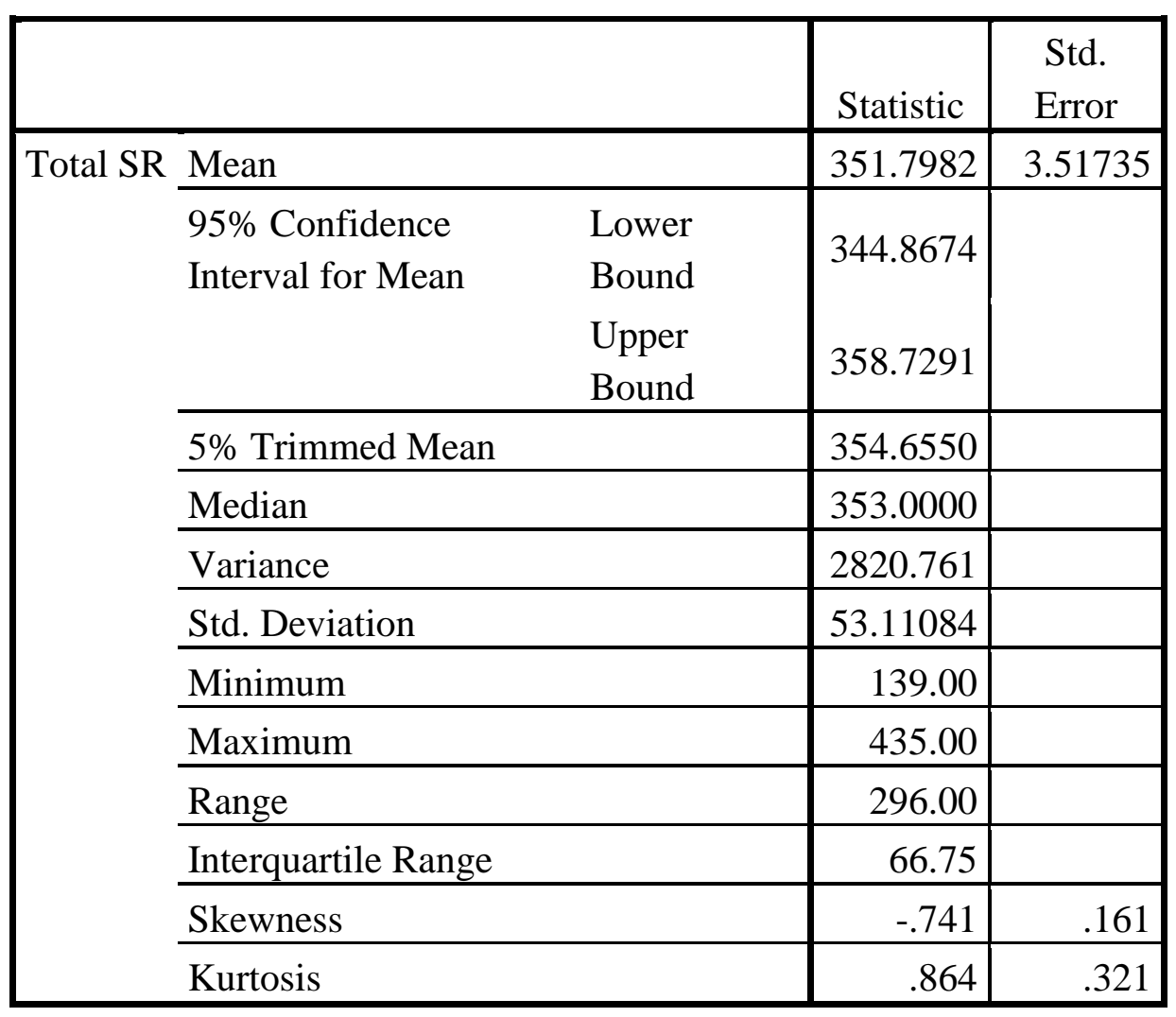

Exploratory Factor Analysis: Determining the Structure of the SRM

Factorability of the data was assessed by the Bartlett's test of sphericity and the Kaiser-Meyer-Olkin (KMO) measure of sampling adequacy. The Bartlett's test was significant $(p \leq .000)$ which indicated that a factor analysis was appropriate. A KMO index of .6 is considered minimum and values closer to 1 are better. The KMO for this data set was .944 which is well above the minimum required. A principal components analysis (PCA) with an oblique rotation and direct oblimin technique using SPSS version 
22 was conducted for the initial factor analysis. Oblique rotations allow components to correlate. A scree test was used to plot each of the eigenvalues of the factors. The scree plot (see Figure 1) was inspected and the change in the shape of the curve indicated that there were two factors that contributed the most to an explanation of the variance in the data. Component 1 accounted for $44.770 \%$ of the variance and Component 2 accounted for $4.031 \%$ with a cumulative of $48.8 \%$ of the variance explained. Component 1 had an eigenvalue of 38.95 and Component 2 had an eigenvalue of 3.507 (see Appendix D, I.).

The oblimin rotation provided a pattern matrix which showed the factor loadings of each of the variables (see Appendix D, II). Of the 87 items, 8 loaded weakly onto both components and 32 loaded below .6 on a single component. These 40 items were excluded from further analysis. Component one included 36 items relating to self-concept and component two had 11 items relating to a sense of community membership that loaded at .6 or higher. There was a strong positive correlation between the two factors ( $r$ $=.675$ ). A total of 47 items (shown in bold in Appendix D, II) were retained for use in a Confirmatory Factor Analysis.

\section{Confirmatory Factor Analysis}

The structure of the measure was assessed through Confirmatory Factor Analysis (CFA) using maximum likelihood estimation as implemented in SPSS AMOS version 19. A total of 47 items were included in the first CFA step and the number of factors was fixed at two. On the two respective factors, 21 items demonstrated low standardized estimates (below .65) at this point and were removed. These items were: 


\section{Component 1: Self}

I can be effective.

I can be aware of my true self.

I can live in the present.

I can make a contribution.

I can be optimistic.

I can make my own decisions.

I look forward to the future.

I can be present instead of anxious.

I know what I want from my future.

I can accept experiences even if they are difficult.

I am responsible for making decisions in my life.

I can express myself.

I am responsible for myself.

I choose which relationships are right for me.

I am more than my experiences with mental health challenges.

I can grow.

I have a future.

I have opportunities to grow.

I have hope for the future.

Component 2: Community

I can be vulnerable. 
I have a supportive group that I do enjoyable things with.

Modification indices were then examined and revealed that the measurement errors for six items were highly correlated with other items. Extreme collinearity can occur when what appear to be separate variables actually measure the same thing (Kline, 2011). Based on the modification indices, the following items were removed:

Component 1: Self

My life is meaningful.

I can exercise my rights.

I can direct my life.

I can set my own goals.

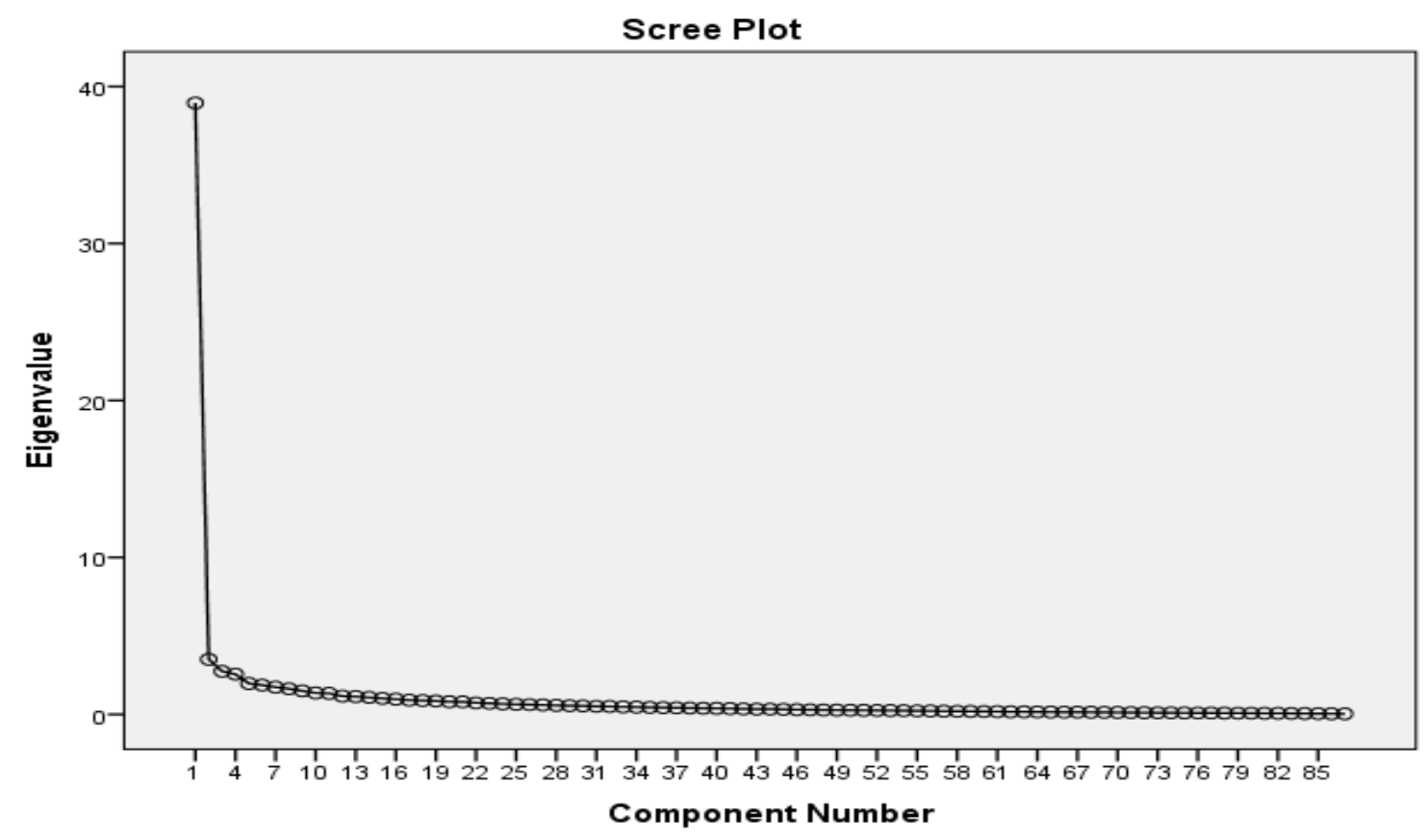

Figure 1: Scree Plot of Eigenvalues of Factors for Exploratory Factor Analysis 


\section{Component 2: Community}

I know others who can understand how my mental health challenges connect to my life experiences.

I have opportunities to talk about how mental health challenges have affected my life.

Modification indices were further examined and revealed some remaining correlation

of error terms between four pairs of items. Co-variances were drawn between the following items:

\section{Component 1: Self}

I plan for my future \& I have inner motivation

I can exercise my personal freedoms \& I am a valuable person

I have strengths \& I have a purpose in life

\section{Component 2: Community}

I have a community that values me \& I have a community that recognizes my abilities

Standardized residual co-variances were then examined for discrepancies between the proposed model and the estimated model. In normal linear models, the standardized residuals have approximately standardized normal distributions: $95 \%$ will be found between -2 and +2 and almost all will fall between -3 and +3 . Large residuals, defined as above an absolute 2.58, indicate bad model fit (Kline, 2011). The item "I have a contribution to make" was found to have a standardized residual co-variance of 2.967 and so was removed to improve model fit. See Table 5 for distribution information on the 
final measure. The factor loadings for the 19 items included in final instrument can be found in Table 6.

Table 5. Final SRM Distribution Data

\begin{tabular}{|c|c|c|c|c|}
\hline & & & Statistic & $\begin{array}{l}\text { Std. } \\
\text { Error }\end{array}$ \\
\hline \multirow[t]{15}{*}{ Total SRM } & \multicolumn{2}{|l|}{ Mean } & 76.9518 & .85026 \\
\hline & \multirow{4}{*}{$\begin{array}{l}95 \% \text { Confidence } \\
\text { Interval for Mean }\end{array}$} & Lower & 752763 & \\
\hline & & Bound & & \\
\hline & & Upper & 78.6272 & \\
\hline & & Bound & & \\
\hline & \multicolumn{2}{|l|}{$5 \%$ Trimmed Mean } & 77.7446 & \\
\hline & \multicolumn{2}{|l|}{ Median } & 78.0000 & \\
\hline & \multicolumn{2}{|l|}{ Variance } & 164.830 & \\
\hline & \multicolumn{2}{|l|}{ Std. Deviation } & 12.83862 & \\
\hline & \multicolumn{2}{|l|}{ Minimum } & 25.00 & \\
\hline & \multicolumn{2}{|l|}{ Maximum } & 95.00 & \\
\hline & \multicolumn{2}{|l|}{ Range } & 70.00 & \\
\hline & \multicolumn{2}{|l|}{ Interquartile Range } & 16.00 & \\
\hline & \multicolumn{2}{|l|}{ Skewness } & -.840 & .161 \\
\hline & \multicolumn{2}{|l|}{ Kurtosis } & 1.013 & .321 \\
\hline
\end{tabular}

The re-specified model of a 19-item instrument (see Figure 2) resulted in a good model fit: $\mathrm{P}$ CMIN/DF $=2.371 ; \mathrm{RMSEA}=.078 ; \mathrm{CFI}=.933 ;$ and $\mathrm{SRMR}=.051$. Attempts to create a shortened instrument resulted in worsened fit indices. The best attempt involved a process in which items were removed due to apparent redundancy and yielded a 16-item instrument with fit indices of: $\mathrm{P}$ CMIN/DF $=2.511$; $\mathrm{RMSEA}=.082$; $\mathrm{CFI}=.932$; and SRMR $=.0522$. Further, an attempt to construct a single factor model also resulted in worsened fit indices: $\mathrm{P}$ CMIN/DF $=3.300 ; \mathrm{RMSEA}=.101 ; \mathrm{CFI}=.900$; 
and SRMR $=.0442$. The two latter fit indices results lend support for accepting the 19item two factor instrument. See Appendix E for the final version of the Social Recovery Measure (SRM). Possible scores range from a low of 19 to a high of 95 with higher scores reflective of better states of social recovery.

Table 6. Factor Loadings for Final SRM Items

\begin{tabular}{|l|l|}
\hline \multicolumn{1}{|c|}{ Self Factor } & \multicolumn{1}{c|}{ Community Factor } \\
\hline I have something to offer others. & I have relationships that are mutually \\
.815 & supportive. \\
I am a capable person. & .848 \\
.808 & I have a community that values me. \\
I plan for my future. & .791 \\
.802 & I feel I belong to a community. \\
I have inner motivation. & .765 \\
.686 & I have a community that recognizes my \\
I can exercise my personal freedoms. & abilities. \\
.780 & .732 \\
I am a valuable person. & I have relationships that inspire hope. \\
.753 & .611 \\
I have strengths. & I have people I can trust. \\
.734 & .662 \\
I have a purpose in life. & I have a supportive group that encourages \\
.645 & me to grow. \\
I can enjoy things I do. & .756 \\
.727 & \\
I have abilities to meet goals. & \\
.714 & \\
I can lead a full life. & Note: 19 item measure with a 5-point \\
.712 & Likert: Strongly Disagree; Disagree; \\
I am valued for who I am. & Neither Disagree of Agree; Agree; \\
.646 & Strongly Agree \\
\hline
\end{tabular}




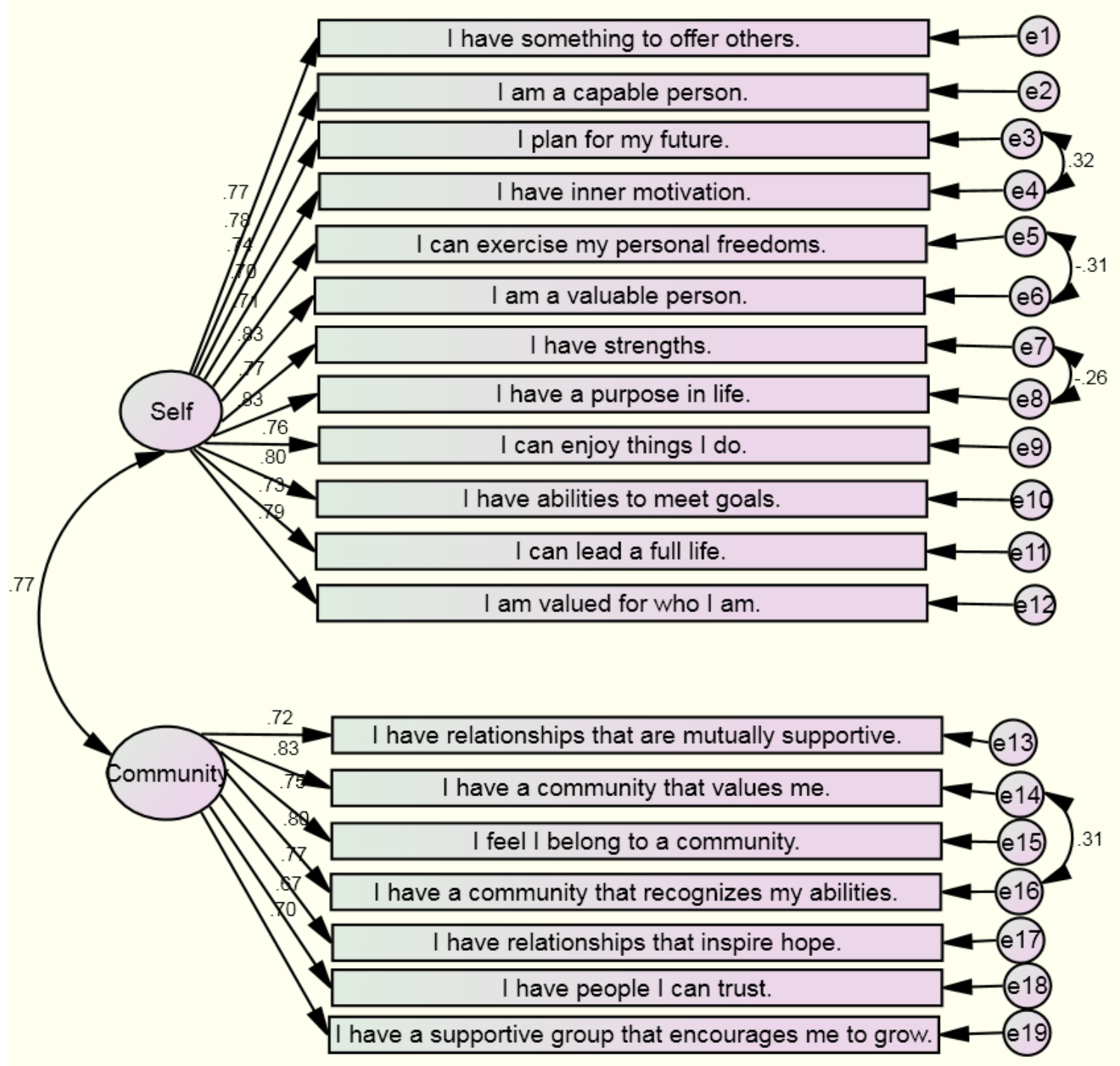

Figure 2: 19-item Two Factor Model of the Social Recovery Measure (SRM) 


\section{Reliability}

Reliability was assessed through Cronbach's alpha coefficient and test-retest procedures. Cronbach's alpha for the Social Recovery Measure as a whole and for the two factors was found to be high. The Social Recovery Measure had Cronbach's alpha coefficient of .951 , the Self factor had $\alpha=.943$, and the Community factor had $\alpha=.890$. Test-retest was conducted with 30 participants who took the measure for a second time two to three weeks after the first administration in order to assess the stability of the instrument. The obtained correlation coefficient was very high, $r=.945, N=30, p \leq .01$ (2-tailed). The standard error of measurement directly estimates the amount of error in the instrument. It is an estimate of the standard deviation of error and can be used to determine what change in scores may be due to error. A standard error of measurement that is $5 \%$ or less of the range of possible scores is recommended. The Self subscale had a SEM of 1.94 (4.04\% of the subscale range) and the Community subscale had a SEM of 1.67 (5.76 of the subscale range) which are considered low (4.75\% of the range of possible scores) and therefore acceptable.

\section{Validity}

Pearson Product Moment Correlations were conducted to assess convergent validity for the Social Recovery Measure (SRM) and the Recovery Assessment Scale (RAS), the 
Questionnaire about the Process of Recovery (QPR), and the Beck Hopelessness Scale (BHS). All correlations were found to be consistent with those hypothesized which provided evidence for the establishment of construct validity. Validity was further assessed through examining differences on the scores on the SRM by participant groups. Recovery Assessment Scale (RAS)

The Social Recovery Measure and the Recovery Assessment Scale were very strongly and positively correlated $(r=.863, p \leq .01)$. The SRM was very strongly correlated with the RAS domains of Confidence and Hope $(r=.820, p \leq .01)$ and Goal and Success Orientation $(r=.811, p \leq .01)$; strongly correlated with the domains of Reliance on Others $(r=.653, p \leq .01)$ and No Domination by Symptoms $(r=.651, p \leq .01)$; and moderately correlated with the domain of Willingness to Ask for Help $(r=.58, p \leq .01)$. The SRM Self domain was very strongly and positively correlated with the RAS domains of Confidence and Hope $(r=.833, p \leq .01)$ and Goal and Success Orientation $(r=.820$, $p \leq .01)$; strongly correlated with the domain of No Domination by Symptoms $(r=.638$, $p \leq .01)$; and moderately correlated with the domains of Willingness to Ask for Help ( $r=$ $.560, p \leq .01)$ and Reliance on Others $(r=.541, p \leq .01)$. The SRM Community domain was strongly and positively correlated with the RAS domains of Confidence and Hope ( $r$ $=.638, p \leq .01)$, Goal and Success Orientation $(r=.638, p \leq .01)$; and Reliance on Others $(r=.706, p \leq .01)$; and moderately correlated with the RAS domains of Willingness to Ask for Help $(r=.506, p \leq .01)$ and No Domination by Symptoms $(r=.545, p \leq .01)$. The Questionnaire about the Process of Recovery $(Q P R)$ 
The Social Recovery Measure and The Questionnaire about the Process of Recovery (QPR) were very strongly and positively correlated $(r=.836, p \leq .01)$. The SRM was very strongly correlated with the QPR Intrapersonal domain $(r=.85, p \leq .01)$ and moderately correlated with the Interpersonal domain $(r=.547, p \leq .01)$. The SRM Self domain was very strongly correlated with the QPR Intrapersonal domain $(r=.833, p \leq$ $.01)$ and moderately correlated with the Interpersonal domain $(r=.539, p \leq .01)$. The SRM Community domain was strongly correlated with the QPR Intrapersonal domain ( $r$ $=.711, p \leq .01)$ and moderately correlated with the Interpersonal domain $(r=.453, p \leq$ $.01)$.

\section{The Beck Hopelessness Scale (BHS)}

The Social Recovery Measure and The Beck Hopelessness Scale (BHS) were strongly negatively correlated $(r=-.643, p \leq .010$. The SRM Self domain and BHS were strongly negatively correlated $(r=-.634, p \leq .01)$ and the Community domain and BHS were moderately negatively correlated $(r=-.531, p \leq .01)$.

\section{Demographic Groups}

A series of one-way between-group analyses of variance were conducted to explore the impact of sexual orientation, relationship status, living situation, and type of treatment engagement on SRM scores. No statistically significant differences were found. A Pearson product-moment correlation coefficient was calculated to explore the impact of education on SRM scores. No statistically significant difference was found. A one-way between-group analysis of variance was conducted to explore the impact of race on SRM scores. No statistically significant differences were found by racial categories as 
originally designated: White, African American, Asian/Pacific Islander, First Nation/Alaskan Native, Latino, and Mixed Race. Race was then recoded into two categories: White and People of color. An independent-samples t-test was conducted and again no statistically significant difference was found (see Table 7).

Pearson product-moment correlation coefficients were calculated to explore the impact of age on SRM scores. There were weak, positive correlation between age and the full SRM, $r=.136, p \leq .043$, and for the Self subscale, $r=.135, p \leq .044$. No statistically significant correlation was found for the Community subscale. A one-way between-group analysis of variance was conducted to explore the impact of gender on SRM scores. No statistically significant differences were found for the full SRM or for the Self subscale. There was a statistically significant difference for the Community subscale: $F(2,219)=$ 3.988, $\mathrm{p} \leq .020$. Post hoc comparisons using the Tukey HSD test indicated that the mean score of females $(M=28.89, S D=5.04)$ was significantly different from the mean score of men $(M=26.91, S D=5.49)$. The effect size as calculated by eta squared was .035 , which is small.

The primary diagnosis groups were changed slightly in the interest of data analysis. $\operatorname{PTSD}(N=15)$ and Anxiety disorder $(N=10)$ were collapsed into one category. Dissociative identity disorder $(N=10)$, Substance use disorder $(N=2)$, Autism spectrum $(N=2)$, Brain injury $(N=1)$, and in mental health recovery/madness $(N=5)$ were collapsed into another single category. A one-way between-groups analysis of variance was conducted to explore the impact of diagnosis on SRM scores. There was a statistically significant difference for the full SRM: F $(5.216)=2.696, p \leq .022$. Post hoc 
comparisons using the Tukey HSD test indicated that the mean score for individuals with a bipolar disorder $(\mathrm{M}=81.05, \mathrm{SD}=10.32)$ was statistically significant different from the mean score for individuals with a diagnosis of depression $(\mathrm{M}=73.34, \mathrm{SD}=14.89)$. The effect size as calculated by eta squared was .058, which is medium. No statistically significant differences were found for either subscales.

One-way between-group analysis of variance tests were conducted to explore the impact of employment on SRM scores. Participant groups included: working full-time, working part-time, student, temporarily unemployed, on disability, retired. There was a statically significant difference for the full SRM: $F(5,216)=4.24, p \leq .001$. The actual difference in mean scores between the groups was medium. The effect size as calculated by eta squared was .089. Post hoc comparisons using the Tukey HSD test indicated that the mean score for the temporarily unemployed group $(M=69.04, S D=14.60)$ was significantly different from the working part time group $(M=81.27, S D=10.69)$ and from the working full time group $(M=81.18, S D=11.23)$. The other groups did not differ significantly from one another. There was a statistically significant differences for the Self subscale scores: $F(5,216)=3.246, p \leq 008$. The effect size as calculated by the eta squared was .069, which is medium. Post hoc comparisons using the Tukey HSD test indicated that the mean score for the working full time group $(M=51.88, S D=6.78)$ was significantly different from the temporarily unemployed group $(M=45.04, S D=10.53)$ and the on disability group $(M=47.58, S D=9.41)$. The effect size as calculated by eta squared was .069 , which is medium. There were statistically significant differences for the Community subscale scores: $F=5,216)=4.764, p \leq .000$. The effect size as 
calculated by eta squared was .09 , which is medium. Post hoc comparisons using the Tukey HSD test indicated that the mean score for the temporarily unemployed group ( $M$ $=24, S D=6.41)$ differed from the working full time group $(M=29.03, S D=4.8)$, the working part time group $(M=30.18, S D=4.08)$, and the on disability group $(M=27.70$, $S D=5.14)$.

Independent-samples t-tests were conducted to compare the SRM scores for participants who took the measure on-line and those who took the measure on paper. There was a statistically significant difference in scores on the full SRM for the on-line group $(M=78.82, S D=12.24)$ and the paper group $(M=74.76, S D=13.22) ; t(226)=$ $2.40, p \leq . .017$. The mean difference in scores was 4.06 with a $95 \%$ confidence interval ranging from .73 and 7.38 . The effect size as calculated by eta squared was .025 , which is small. There was a statistically significant difference in scores on the Self subscale for the on-line group $(M=50.29, S D=8)$ and the paper group $(M=47.25, S D=8.91) ; t(226)=$ $2.717, p \leq .007$. The mean difference in self subscale scores was 3.04 with a confidence interval ranging from .836 and 5.25. The effect size as calculated by eta squared was .032, which is small. No statistically significant difference in Community subscale scores was found.

One-way between-group analysis of variance tests were conducted to explore the impact of participant state of residency on SRM scores. Participant groups were: Oregon, Out of State, and Unknown. There was a statistically significant difference in SRM scores for the three groups: $F(2,225)=4.07, p \leq .018$. Despite reaching statistical significance, the actual difference in mean scores between the groups was small. The 
effect size, calculated using eta squared, was .035. Post-hoc comparisons using Tukey HSD indicated that the mean score for the Out of State group $(M=80.64, S D=10.88)$ was significantly different from the Oregon group $(M=75.35, S D=1.07)$. The Unknown group did not differ significantly from Oregon or Out of State groups. There was a statistically significant difference in Self subscale scores for the three groups: $F(2,225)$ $=3.984, p \leq .020$. The effect size as calculated by eta squared was .020 , which is small. Post-hoc comparisons using Tukey HSD indicated that scores for the Out of State group $(M=51.32, S D=6.89)$ was statistically different from the Oregon group $(M=47.84, S D$ =9.01). The Unknown group did not differ significantly from the Oregon or Out of State groups. No statistically significant difference in the Community subscale scores was found.

Table 7. Correlation, One-way ANOVAs, and T-Test of Non-significant Findings

\begin{tabular}{|l|l|l|l|}
\hline Demographic & $\mathbf{N = 2 2 2}$ & $\mathbf{M}$ & SD \\
\hline $\begin{array}{l}\text { Education } \\
r=.008, p=.238\end{array}$ & & 76.95 & 12.84 \\
\hline $\begin{array}{l}\text { Sexual Orientation } \\
F(2,219)=.154, p= \\
.858\end{array}$ & & & \\
\hline Straight/Heterosexual & 171 & & \\
\hline LGBTQP, Asexual & 34 & 76.83 & 12.42 \\
\hline Prefer not to answer & 15 & 76.75 & 13.23 \\
\hline $\begin{array}{l}\text { Relationship Status } \\
F(4,217)=1.56, p= \\
.170\end{array}$ & & 78.73 & 17.24 \\
\hline Single & & & \\
\hline In a relationship(s) & 44 & & 12.34 \\
\hline Married/Domestic & 38 & 75.12 & 12.98 \\
\hline Separated & 29 & 79.34 & 11.83 \\
\hline Widowed & 6 & 80.07 & 14.93 \\
\hline
\end{tabular}




\begin{tabular}{|c|c|c|c|}
\hline Demographic & $\mathrm{N}=222$ & $\mathbf{M}$ & SD \\
\hline \multicolumn{4}{|l|}{$\begin{array}{l}\text { Living Setting } \\
F(3,218)=.745, p= \\
.526\end{array}$} \\
\hline Urban & 103 & 76.66 & 13.85 \\
\hline Small Town & 51 & 76.09 & 11.16 \\
\hline Suburb & 56 & 78.91 & 12.43 \\
\hline Rural/Frontier & 12 & 73.91 & 13.23 \\
\hline \multicolumn{4}{|l|}{$\begin{array}{l}\text { Type of Treatment } \\
\text { Engagement } \\
F(2,219)=2.168, p \\
=.117\end{array}$} \\
\hline $\begin{array}{l}\text { Traditional } \\
\text { Community Mental } \\
\text { Health } \\
\text { (including primary } \\
\text { care, private mental } \\
\text { health care, and } \\
\text { working as a } \\
\text { provider) }\end{array}$ & 43 & 74.32 & 12.93 \\
\hline $\begin{array}{l}\text { Peer Services or } \\
\text { Supports }\end{array}$ & 34 & 80.44 & 10.91 \\
\hline $\begin{array}{l}\text { Both Traditional and } \\
\text { Peer Services }\end{array}$ & 145 & 76.91 & 13.16 \\
\hline \multicolumn{4}{|l|}{$\begin{array}{l}\text { Race/Ethnicity } \\
F(5,216), p=.154\end{array}$} \\
\hline White & 171 & 76.70 & 12.30 \\
\hline African American & 14 & 81.21 & 12.46 \\
\hline $\begin{array}{l}\text { Asian/Pacific } \\
\text { Islander }\end{array}$ & 8 & 79.37 & 10.21 \\
\hline $\begin{array}{l}\text { First Nation/Native } \\
\text { American/Alaskan }\end{array}$ & 3 & 68.00 & 18.52 \\
\hline Latino & 5 & 65.40 & 26.46 \\
\hline Mixed Race & 21 & 79.23 & 12.86 \\
\hline \multicolumn{4}{|l|}{$\begin{array}{l}\text { Independent } \\
\text { Samples t-test for } \\
\text { Race } \\
t(220)=.526, p= \\
.166 \\
\text { Mean Difference }= \\
1.08 \\
95 \% \text { CI: }-5.13 \text { to } 2.97\end{array}$} \\
\hline White & 171 & 76.70 & 12.30 \\
\hline People of Color & 51 & 77.78 & 14.71 \\
\hline
\end{tabular}




\section{Chapter 5}

\section{DISCUSSION}

The purpose of this research project was to develop the Social Recovery Measure (SRM) and evaluate its psychometric properties. This chapter will briefly present the rationale and theoretical framework for the study, review the methodology, and summarize and discuss the research findings. Strengths and limitations of the research will be explored as will recommendations for future research and implications for social work practice.

\section{Summary}

Mental health recovery is a complex phenomenon involving clinical, functional, physical, existential, and social dimensions. The social dimension has been said to involve meaningful relationships and integration with supportive individuals and a wider community (Whitley \& Drake, 2010). The Social Recovery Measure (SRM) was developed to create a self-administered instrument with a specific focus on the social dimension. Social recovery is highly relevant for social work given the discipline's commitment to disenfranchised populations and investment in creating enabling social environments. The study was grounded in disability and mad theories which locate disability at the intersection of the person and the environment. No other recovery measure was found that was grounded in the disability or mad theory bases. The theoretical stance calls for social acceptance and the valuing of diverse human experiences. Physiological and psychological realities are not denied but understood to be experienced in social contexts that impact mental states. Recovery can be conceptualized 
as relational and reliant on opportunities for social engagement. While recovery includes a number of dimensions and definitions, individuals with lived experience of mental health challenges have asserted that core aspects include a strong self-concept and a worthwhile life. As explored in Chapter 2, the recovery outcome literature includes findings that greater involvement in education, work, and social pursuits is associated with better recovery. Recovery involves affiliation and leading a purposeful life. It is not just an individual phenomenon but is dependent on social resources and opportunities.

\section{Methodology}

The study involved the facilitation of six focus groups with a total of 41 individuals in recovery from mental health challenges. The transcribed focus groups were subjected to a thematic analysis which resulted in a 32-item draft measure of social recovery. The three initial domains concerned a sense of community involvement, a positive selfconcept, and personal capacities or intrapersonal skills. An expert panel of four individuals in academia who specialize in mental health recovery was enlisted at this point and provided guidance throughout the project. The expert panel focused particularly on content validity. A second 73-item version of the measure was drafted in order to include items that addressed nuance or gradation of meaning and to incorporate refinement of the wording of items by the expert panel. The second draft measure was reviewed by an additional five-member focus group followed by cognitive interviews with five different individuals receiving concurrent community mental health and peer services who pilot-tested the measure to ensure that items reflected the underlying construct of social recovery and were worded effectively. The resulting third draft 
measure consisted of 87 items and was taken by 228 individuals involved in community treatment and/or peer services who also completed the Recovery Assessment Scale (RAS), the Questionnaire about the Process of Recovery Questionnaire (QPR), and the Beck’s Hopelessness Scale (BHS).

Factor Solution and Findings

A major assumption of structural equation modeling is normal distribution of data. Skewness and kurtosis for the draft measure as a whole and for each item were well within acceptable ranges by conservative guidelines. Normality of the sample distribution was supported and structural equation modeling was conducted. An exploratory factor analysis determined a two-factor structure for the measure. Factors were named "Self" and "Community." Separate domains of self-concept and personal capacities as derived by the thematic analysis of focus group data were not supported as items loaded onto the same factor. A confirmatory factor analysis resulted in a 19-item instrument with good model fit: $\mathrm{P}$ CMIN/DF $=2.371 ; \mathrm{RMSEA}=.078 ; \mathrm{CFI}=.933$, and $\operatorname{SRMR}=.040$. Reliability assessed through Cronbach's alpha coefficient for the measure was high at .951 with a Self factor alpha of .943 and a Community factor alpha of .890 . A test-retest reliability analysis with 30 individuals resulted in a very high correlation coefficient of .945. The calculated SEM of 1.94 for the Self and 1.67 for the Community domain was low, indicating that changes in scores were unlikely to be due to measurement error. The SEM values determined that there was little error in the SRM. Convergent validity was demonstrated through very strong positive correlations between the SRM and the RAS and QPR and a strong negative correlation with the BHS. No 
statistically significant differences among demographic groups on the SRM were noted except for a moderate difference for temporarily unemployed status, small differences for on-line compared to paper participation, and participation by Oregon residents compared to individuals living out of the state. These differences will be explored briefly in the following discussion section.

\section{Discussion}

The Social Recovery Measure was developed in collaboration with individuals in recovery. Items were constructed from direct exploration of their lived experience and were refined through their scrutiny. Therefore, the measure should appear valid to individuals to whom it will be administered and can be considered to have strong face validity. It can be anticipated that the SRM will be well-received by individuals who complete it. The development of the SRM through an iterative process with individuals in recovery and those recognized as experts in the academic field supports content validity. Items were closely reviewed for linkage to and coverage of the social recovery construct.

Individuals with lived experience are noted to not limit recovery to the absence of symptoms or the reduction of use of services. Their accounts of recovery emphasize the key roles played by community connections, hope, a positive identity, meaningful life and social roles, and empowerment (Gordon, Ellis, Siegert, \& Walkey, 2013). As briefly reviewed in Chapter 1, I identify as being in recovery from bipolar disorder and have benefited from peer support. I disclosed my identity to potential research participants at every stage of the project including recruitment to focus groups and cognitive interviews. My recovery status was known to consumer gatekeepers which allowed me entry into the 
program sites that I might not otherwise have been able to gain. My interactions with consumer program managers and participants featured a mutuality of identity that appeared to build a sense of trust and support engagement. Focus groups and cognitive interviews were influenced by this sense of mutuality and my lived experience allowed me to have a strong appreciation for the experiences of participants and the importance they gave to particular recovery processes. Due to my lived experience, I was able to develop a felt sense for the experiences the participants related, share how I connected to what they had expressed, and ask experientially informed and nuanced follow-up questions. Three of the four members of the expert panel had lived experience as well. The rapport among the individuals was high and the dialogue was quite productive. These expert panel members were reviewing items from the perspective of lived experience as well as academically in a manner that matched mine. Shared experiences facilitated an easy flow to the process and allowed me to better receive challenges to items as supportive of the task at hand rather than as overly critical.

Skewness and kurtosis assessment established normality of the sampling distribution and met a major assumption of structural equation modeling. The conduct of a factor analysis was supported by the finding of normality of the sampling distribution. Factor analysis concerns the modeling of observed variables as a function of a latent variable that is unobserved. It allows for the determination of the number of factors that underlie a set of items and assists with identification of item performance. An exploratory factor analysis determined a two-factor structure and a confirmatory factor analysis provided evidence of good model fit for the factor structure. 
The construct validity of the resulting 19-item Social Recovery Measure was demonstrated through convergent testing with established measures broadly used in the field. A measure should significantly correlate with established instruments that measure related constructs. The SRM was very strongly correlated with the Recovery Assessment Scale (RAS) and the Questionnaire about the Process of Recovery (QPR). The finding of only a moderate correlation with the Willingness to Ask for Help domain of the RAS was expected as items of the SRM do not concern seeking assistance or treatment but rather concern the development of a strong self-concept and a sense of social inclusion. Similarly, the finding of only a moderate correlation with the Interpersonal domain of the QPR was expected as the particular domain items concern making treatment decisions and engaging in interpersonal advocacy for systems change, not development of a strong self-concept and sense of social inclusion. The SRM and the Beck Hopelessness Scale were strongly negatively correlated. A sense of being a valuable individual with something to offer to a community is inconsistent with a feeling of hopelessness.

Construct validity of the SRM was further supported by non-significant findings between SRM scores and variables of race, sexual orientation, relationship status, living situation, education, and type of treatment engagement. The SRM did not discriminate among these factors and appeared to function well for individuals of different social categories, identities, lived experiences, and contexts. The measure demonstrated that it may be used with the range of adult consumers of community mental health treatment and peer supports. Administration need not be confined to individuals with only certain mental health presentations or valued social identities. This matter is highly important 
given the diversity among individuals with lived experience of mental health challenges who find themselves in need of assistance and community inclusion.

The Pearson product-moment correlation of $r=.136, p \leq .043$ for age and the full measure is so weak as to be without practical significance. However, this variable should be included in future investigations of how the SRM functions with the targeted population. Future psychometric testing should make use of samples that include a range of ages as represented in the general population. The statistically significant difference in Community subscale scores by gender may hold greater meaning. As noted in the last chapter, females demonstrated higher scores on the Community subscale compared to males. While the difference was statistically significant, the mean difference was 1.98 points and the effect size was small, .035 eta squared. However, most studies have found that females develop mental health conditions later than men, tend to have established higher levels of social support before illness onset, and present with better social functioning than males throughout the life course (Ochoa, Usall, Cobo, Labad, \& Kulkarni, 2012). It may be that the SRM scores of females will prove to be slightly higher than the mean scores of males as the measure is implemented. This matter should be further explored in future psychometric testing of the SRM.

The statistically significant difference found for full SRM scores by diagnosis is intriguing. As previously noted, individuals diagnosed with bipolar disorder $(M=81.05$, $S D$ 10.32) demonstrated higher scores than individuals diagnosed with depression $(M=$ $73.74, S D=14.89$ ). The effect size by eta squared was .058 , which is medium. No other statistically significant differences by diagnosis were found. Individuals diagnosed with 
bipolar disorder are known to experience increased self-confidence, energy levels, and social interest during hypomania and mania states. By contrast, depressed states are known to feature sensitivity and social timidity and withdrawal (Akiskal et al., 2006; Kiejna et al., 2006). The difference in mean scores between these two groups may be accounted for by these differences in mood states. This finding should be explored in future psychometric testing of the SRM. It may be that on average individuals diagnosed with depression require more attention to their social recovery processes as compared to individuals with bipolar diagnoses.

The statistically significant differences in SRM scores for the three areas of employment status, on-line versus paper administration, and state of residency merit some discussion. As noted previously, individuals who were temporarily unemployed demonstrated lower full SRM scores compared to individuals who were working part time and full time. The economy is known as one of the most important social environments that affects individual well-being and the economic stressor of job loss carries a pronounced social cost (Dooley, 2003). The benefits of workforce participation for mental health are established and involve a number of aspects of someone's life including social role, self-esteem, and sense of purpose (Huxley, 2001; Olesen, Butterworth, Leach, Kelaher, \& Pirkis, 2013). It is little wonder that participants who were temporarily without work roles exhibited lower social recovery scores. A measure sensitive to this type of change given the pronounced influence of employment on social status would have utility in service settings for assessing recovery and constructing care plans. 
Participants who took the SRM on-line scored higher $(M=78.82, S D=12.24)$ than those who took the measure on paper $(M=74.76, S D=13.22)$. The small (eta squared $=$ .025) although statistically significant difference in SRM scores by on-line versus paper mode of administration may be due to an unexplored association between computer literacy and social recovery. Out-of-state participants who took the SRM scored higher $(M=80.64 S D=10.88)$ than Oregon participants $(M=75.35 S D=1.07)$. It could be conjectured that the small (eta squared $=.035$ ) although statistically significant difference in SRM scores by state of residency was due to the higher level of trust or motivation required to engage in a study conducted by a researcher unfamiliar to the participant. However, both differences in means were small and may have been simply artifacts of no practical importance. Given how small the differences were for mode of participation and state of residency, it is anticipated that the SRM can be administered in different formats and to a range of participants to good effect. As previously reviewed, validity testing established that the SRM performs consistently with well-regarded recovery instruments in wide use.

The SRM was found to have excellent reliability. The Cronbach's alpha coefficient of .951 demonstrates that the individual items are highly intercorrelated and strongly relate to the underlying construct. The strong internal consistency indicates that variance in SRM scores will be attributable to changes in social recovery rather than due to random error. The very strong correlation coefficient $(r=.945)$ for the test-retest procedure provides evidence that the SRM performs in a consistent manner over time as scores should not change unless there is an actual change in social recovery. 
Items of the SRM reflect social recovery elements of experiencing meaningful relationships, a sense of being able to make a social contribution, and a sense of belonging. The 19 items readily map onto the CHIME conceptual framework of recovery processes (Leamy, Bird, Le Boutillier, Williams, \& Slade, 2011):

Category 1: Connectedness

I have a community that values me.

I feel I belong to a community.

I have a community that recognizes my abilities.

I have people I can trust.

I have a supportive group that encourages me to grow.

Category 2: Hope and optimism about the future

I plan for my future.

I have inner motivation.

I have relationships that inspire hope.

Category 3: Identity

I have something to offer others.

I am a capable person.

I am a valuable person.

Category 4: Meaning in life

I have a purpose in life.

I can enjoy things I do.

I can lead a full life. 
I am valued for who I am.

I have relationships that are mutually supportive.

\section{Category 5: Empowerment}

I can exercise my personal freedoms.

I have strengths.

I have abilities to meet goals.

The Social Recovery Measure has been found to be a psychometrically robust instrument and use is indicated for a broad range of settings. First, it can be used in practice and evaluation. The measure was developed and refined with significant consumer direction. It reflects a positive frame on recovery as items speak to the presence of capacities and supports. It is designed for self-administration, items are worded positively for clarity and ease of use, and it is relatively brief at 19 items. The SRM could be employed in community mental health treatment and peer support contexts for individual assessment and goal setting. Aggregated data could be employed as part of program and service system evaluation. The SRM reflects social work values of the dignity and worth of the person and the importance of human relationships and social work principles of individual well-being in a social context. It was designed to be used by a range of support and treatment providers. It could readily be incorporated into the array of instruments currently used by case managers, therapists, and program supervisors. Community psychologists and applied sociologists could be expected to be naturally drawn to use of the SRM. It is hoped that consumer-run programs will gravitate towards use of the SRM given that it was developed by someone who openly identifies as 
being in recovery who incorporated the testimony of participants in recovery. Consumer operated programs are currently expanding and the SRM could be incorporated in developing the evidence base for such operations and delineating the effective components.

Secondly, this measure can be used in research and the development of theory. Recovery as a multidimensional construct must be specifically delineated so as to avoid ambiguity and conceptual conflation. "Recovery" can be used to refer to matters as different as symptomatic resolution and occupation of valued social roles. The recovery model developed from a movement led by consumers and survivors of the mental health system to promote hope, self-determination, and social inclusion, while the clinical aspects of recovery have dominated mental health research and practice. However, there is a developing appreciation for the impact of the social environment. Mental health and illness are manifested in social contexts that can function to enable or disable the individual. The under-investigated area of social recovery calls for focused study and analysis including use of developed instruments. Psychometrically sound measurement of social recovery is required in order for the subjective concept to take on an objective reality and impact services and supports.

\section{Limitations}

The major limitation of the study concerns the sample size, type, and composition. While the sample of 228 well exceeds minimal requirements, a larger sample size would have been better for conducting a confirmatory factor analysis. Factorial validity will need to be replicated in future studies. The non-probability, purposive nature of the 
sample limits external validity of the findings as it may not be representative of the target population. For instance, $70 \%$ of participants lived in the state of Oregon. Participants were individuals already engaged in services or supports of some type. All participants were volunteers and the extent to which participants may have differed from those who declined to participate cannot be known. The study sample was 59\% female, 5\% transgender, and $36 \%$ male, whereas the population as a whole is roughly $50 \%$ male. Further, only $23 \%$ of participants identified as people of color compared to $28 \%$ of the US population as a whole. The percentage of participants who identified as AfricanAmerican was $6 \%$ whereas $13 \%$ of the US population identifies as African-American. Similarly, the percentage of participants who identified as Latino was only $2 \%$ compared to the US population which is $17 \%$ Latino. By contrast, $10 \%$ of participants identified as mixed race compared to $2.5 \%$ of the US population. Overall, the low number of participants in specific racial and ethnic categories limited analysis of measure performance. Further, only $18 \%$ of participants were between the ages of 18 and 34 compared to $23.5 \%$ of the US population which may limit applicability of the measure to younger individuals. The sample was also fairly educated with $44 \%$ having a bachelors or graduate degree compared to $30 \%$ of the US population aged 25 or older (US Census, 2014). However, the level of education was counterbalanced by the fact that only $39 \%$ of the sample was employed full-time or part-time. Data on income were not gathered so it is not known if social recovery scores may have varied by access to material resources.

Only information on primary diagnosis was gathered so it is not known how many individuals may have had co-occurring mental health or substance use disorders. More 
complicated diagnostic experiences are known for making recovery more problematic. Similarly, physical health and disability data were not gathered and it is not known if such conditions may have led to significant differences in SRM scores. At least some potential participants objected to the diagnostic language used. The objections were communicated directly to the researcher either in-person or by emails sent by individuals who had considered engaging in the study. While the number of individuals was relatively small, the group included established consumer leaders who may function as gatekeepers to potential research participants. Language of the medical model is perceived by some in the consumer community as un-affirming as it does not reflect their lived experience with mental distress in social context. It may be wise to consider using more affirming language such as "extreme states of consciousness" or "voices and visions" in future studies. Finally, predictive validity, the extent to which performance on the SRM relates to actual later performance of social recovery, was not established. It was infeasible to follow participants over extended periods given time constraints.

\section{Recommendations and Implications}

Future research should consist of additional testing with diverse samples of adults in recovery from mental health challenges drawn from a number of locations. Samples should be larger to support more definitive confirmatory factor analysis testing. More specifically, samples should include a greater percentage of younger adults and people of color and an equal proportion of men and women. It would be useful to include variables of income, co-occurring mental health conditions and substance use disorders, and physical health and disability conditions. Such testing will improve the generalizability of 
the measure. Ongoing use and testing may provide additional support for reliability of the measure. Construct validity for the measure is strongly supported by the study, but could obtain additional support through future validation studies. Predictive validity of the SRM has yet to be investigated. Social scientists from a number of disciplines conduct disability and mad research. The area of investigation is notable for its interdisciplinary nature as researchers may be in social work, clinical or community psychology, disability studies, and public health. Therefore, future research should involve multidisciplinary teams. The SRM could serve to strengthen the theoretical underpinning of the development of interventions premised on the social recovery construct.

\section{Implications}

The SRM has been shown to be a reliable and valid measure of social recovery. It demonstrated promise as a measure for practice and program evaluation as well as in research and theoretical development. It could be suitably employed in comparing interventions and the performance of different programs. The SRM could assist with discerning what needs to be addressed in treatment and support services to best serve an individual. Development of interventions premised on the importance of social recovery will offer social work a means of practice that is consistent with the values and principles of the discipline. The SRM could be used as a non-clinical diagnostic measure and would be suitable for use in consumer-run programs that deemphasize a focus on symptoms while placing more importance on peer relationships and social opportunities. Results would be useful in determining what aspects of social recovery are present and what aspects call for support. Based on individual outcomes, the social worker could 
work on cultivating certain opportunities or environments in order to enhance progress. Social work is expected to provide empirical support for interventions and build evidence-based practices. To evaluate the effectiveness of practice and programs, social workers need to utilize measures with strong psychometric properties such as the Social Recovery Measure.

Further research in social recovery could test the theory base underlying responses to the experience of mental distress and psychiatric disability. Medical or psychological approaches to recovery lead to particular predictions whereas social approaches call for still other explorations. Mad theory is still in a developmental stage and requires investigation through generation of hypotheses subjected to rigorous testing. The differential effects of the dimensions of recovery remain unclear and could be anticipated to function in various ways depending on the particular individual at a specific time and environment. There is a need to better understand the relationship between social recovery and other aspects of recovery in order to determine what helps and what hinders recovery processes. Future research on social recovery would lead to a greater appreciation for what is helpful to whom, under what conditions, and why. Data on social recovery could be used to develop policy in support of social inclusion. 


\section{REFERENCES}

Abell, N., Springer, D. W., \& Kamata, A. (2009). Developing and validating rapid assessment instruments. New York: Oxford University Press.

Adame, A. (2012). The disappointed evangelist: Or, how I learned to stop worrying and love ambiguity. Reflective Practice, 13(4), 597-607.

Adame, A. L., \& Knudson, R. M. (2007). Beyond the counter-narrative: Exploring alternative narratives of recovery from the psychiatric survivor movement. Narrative Inquiry, 17(2), 157-178.

Akiskal, H. S., Kilzieh, N., Maser, J. D., Clayton, P. J., Schettler, P. J., Shea, M. T., ... \& Keller, M. B. (2006). The distinct temperament profiles of bipolar I, bipolar II and unipolar patients. Journal of Affective Disorders, 92(1), 19-33.

Almedom, A. M. (2005). Social capital and mental health: An interdisciplinary review of primary evidence. Social Science \& Medicine, 61(5), 943-964.

Amador, X. F., \& Strauss, S. A. (1993). Scale to Assess Unawareness of Mental Disorders. New York: Human Sciences.

American Psychiatric Association. (2013). Diagnostic and statistical manual of mental disorders (DSM-5®). Washington, DC: American Psychiatric Publishers.

Anastasiou, D., \& Kauffman, J. M. (2013). The social model of disability: Dichotomy between impairment and disability. Journal of Medicine and Philosophy, 38(4), 441459.

Andresen, R., Caputi, P., \& Oades, L. (2006). Stages of recovery instrument: Development of a measure of recovery from serious mental illness. Australian and 
New Zealand Journal of Psychiatry, 40(11-12), 972-980.

Armstrong, N. P., Cohen, A. N., Hellemann, G., Reist, C., \& Young, A.S.

(2014).Validating a brief version of the mental health recovery measure for individuals with schizophrenia. Psychiatric Services, 65(9), 1154-1159.

Åsberg, M., \& Schalling, D. (1979). Construction of a new psychiatric rating instrument, the Comprehensive Psychopathological Rating Scale (CPRS). Progress in Neuro Psychopharmacology, 3(4), 405-412.

Austin, S. F., Mors, O., Secher, R. G., Hjorthøj, C. R., Albert, N., Bertelsen, M., Jensen, H., Jeppesen, P., Petersen, L., Randers, L., Thorup, A, \& Nordentoft, M. (2013). Predictors of recovery in first episode psychosis: The OPUS cohort at 10 year followup. Schizophrenia Research, 150(1), 163-168.

Bajekal, M., \& Purdon, S. (2001). Social capital and social exclusion: Development of a condensed module for the Health Survey for England. London: National Centre for Social Research.

Barber, R., Beresford, P., Boote, J., Cooper, C., \& Faulkner, A. (2011). Evaluating the impact of service user involvement on research: A prospective case study. International Journal of Consumer Studies, 35(6), 609-615.

Barker, P., \& Buchanan-Barker, P. (2010). The tidal model of mental health recovery and reclamation: Application in acute care settings. Issues in Mental Health Nursing, 31(3), 171-180.

Barnes, H. (2011). Does mental illness have a place alongside social and recovery models of mental health in service users' lived experiences? Issues and implications for mental 
health education. The Journal of Mental Health Training, Education and Practice, $6(2), 65-75$.

Battles, H. (2012). Towards Engagement: Exploring the prospects for an integrated anthropology of disability. vis-à-vis: Explorations in Anthropology,11(1), 107-124.

Baumann, A. E. (2007). Stigmatization, social distance and exclusion because of mental illness: The individual with mental illness as a "stranger". International Review of Psychiatry, 19(2), 131-135.

Baumgartner, J. N., \& Burns, J. K. (2013). Measuring social inclusion—a key outcome in global mental health. International Journal of Epidemiology, 43, 354-364.

Beck, A. T., Weissman, A., Lester, D., \& Trexler, L. (1974). The measurement of pessimism: The Hopelessness Scale. Journal of Consulting and Clinical Psychology, 42(6), 861-865.

Bellack, A. S. (2006). Scientific and consumer models of recovery in schizophrenia: Concordance, contrasts, and implications. Schizophrenia Bulletin, 32(3), 432-442.

Bellack, A. S., \& Drapalski, A. (2012). Issues and developments on the consumer recovery construct. World Psychiatry, 11(3), 156-160.

Bengtsson-Tops, A., \& Svensson, B. (2010). Mental health users' experiences of being interviewed by another user in a research project. A qualitative study. Journal of Mental Health, 19(3), 234-242.

Beresford, P. (2005). Social work and a social model of madness and distress. Social Work and Social Sciences Review, 12(2), 59-73.

Beresford, P. \& Campbell, P. (2005). Madness and mental distress. In Gary L. Albrecht 
(Ed). Encyclopedia of disability (pp. 1049-1053). Thousand Oaks, CA: SAGE.

Bergstresser, S. M., Brown, I. S., \& Colesante, A. (2013). Political engagement as an element of social recovery: A qualitative study. Psychiatric Services,64(8), 819-821.

Birchwood, M., Smith, J. O., Cochrane, R., Wetton, S., \& Copestake, S. (1990). The Social Functioning Scale. The development and validation of a new scale of social adjustment for use in family intervention programmes with schizophrenic patients. The British Journal of Psychiatry,157(6), 853-859.

Bluebird, G. (2014). History of the Consumer/Survivor Movement. Retrieved from http://nationalempowermentcenter.com/articles.

Bolier, L., Haverman, M., Westerhof, G. J., Riper, H., Smit, F., \& Bohlmeijer, E. (2013). Positive psychology interventions: A meta-analysis of randomized controlled studies. BMC Public Health, 13(1), 1-20.

Bolt, D. (2005). From blindness to visual impairment: Terminological typology and the social model of disability. Disability \& Society, 20(5), 539-552.

Borg, M., \& Davidson, L. (2008). The nature of recovery as lived in everyday experience. Journal of Mental Health, 17(2), 129-140.

Borthwick, A., Holman, C., Kennard, D., McFetridge, M., Messruther, K, \& Wilkes, J. (2001). The relevance of moral treatment to contemporary mental health care. Journal of Mental Health, 10(4), 427-439.

Bottlender, R., Strauß, A., \& Möller, H. J. (2010). Social disability in schizophrenic, schizoaffective and affective disorders 15years after first admission. Schizophrenia Research, 116(1), 9-15. 
Bowling, A. (2009). Research methods in health: Investigating health and health services. New York: McGraw-Hill International.

Bradshaw, W., Armour, M. P., \& Roseborough, D. (2007). Finding a place in the world: The experience of recovery from severe mental illness. Qualitative Social Work, 6(1), 27- 47.

Braslow, J. T. (2013). The manufacture of recovery. Annual Review of Clinical Psychology, 9, 781-809.

Bromley, E., Gabrielian, S., Brekke, B., Pahwa, R., Daly, K. A., Brekke, J. S., \& Braslow, J. T. (2013). Experiencing community: Perspectives of individuals diagnosed as having serious mental illness. Psychiatric Services, 64(7), 672-679.

Brown, L., \& Boardman, F. K. (2011). Accessing the field: Disability and the research process. Social Science \& Medicine, 72(1), 23-30.

Brownell, T., Schrank, B., Jakaite, Z., Larkin, C., \& Slade, M. (2015). Mental health service user experience of positive psychotherapy. Journal of Clinical Psychology, 71(1), 85-92.

Brown, L. D., Shepherd, M. D., Merkle, E. C., Wituk, S. A., \& Meissen, G. (2008). Understanding how participation in a consumer-run organization relates to recovery. American Journal of Community Psychology, 42(1-2), 167-178.

Bureau of Labor Statistics (2011). Current population survey. Retrieved from http://www.bls.gov/cps.

Campbell, F. A. K. (2008). Exploring internalized ableism using critical race theory. Disability \& Society, 23(2), 151-162. 
Campbell-Orde, T., Chamberlin, J. C., Carpenter, J., \& Leff, H. S. (2005). Measuring the promise: A compendium of recovery measures, Volume II. Cambridge, MA: Evaluation Center of the Human Services Research Institute.

Carey, M. (2011). Should I stay or should I go? Practical, ethical and political challenges to "service user" participation within social work research. Qualitative Social Work, 10(2), 224-243.

Carlson, J. A., Sarkin, A. J., Levack, A. E., Sklar, M., Tally, S. R., Gilmer, T. P., \& Groessl, E. J. (2011). Evaluating a measure of social health derived from two mental health recovery measures: The California Quality of Life (CA-QOL) and Mental Health Statistics Improvement Program Consumer Survey (MHSIP).Community Mental Health Journal, 47(4), 454-462.

Center for Psychiatric Rehabilitation (2012). What is psychiatric disability and mental illness? Retrieved from http://www.bu.edu/cpr/reasaccom/whatis-psych.html.

Centers for Disease Control and Prevention (2011). Mental illness surveillance among adults in the United States. Morbidity and Mortality Weekly Report, 60, 2-29.

Chamberlin, J. (1990). The ex-patients' movement: Where we've been and where we're going. Journal of Mind and Behavior, 11(3), 323-336.

Chen, E. Y., Tam, D. K., Wong, J. W., Law, C. W., \& Chiu, C. P. (2005). Selfadministered instrument to measure the patient's experience of recovery after firstepisode psychosis: Development and validation of the Psychosis Recovery Inventory. Australian and New Zealand Journal of Psychiatry, 39(6), 493-499. Cherney, J. L. (2011). The rhetoric of ableism. Disability Studies Quarterly,31(3). 
Cockerham, W. C. (1992). Sociology of mental disorder. Englewood Cliffs, NJ: Prentice Hall.

Cohen, A., Patel, V., Thara, R., \& Gureje, O. (2008). Questioning an axiom: Better prognosis for schizophrenia in the developing world? Schizophrenia Bulletin, 34(2), 229-244.

Cohen, C. I., Pathak, R., Ramirez, P. M., \& Vahia, I. (2009). Outcome among community dwelling older adults with schizophrenia: Results using five conceptual models. Community Mental Health Journal, 45(2), 151-156.

Cohen, O. (2005). How do we recover? An analysis of psychiatric survivor oral histories. Journal of Humanistic Psychology, 45(3), 333-354.

Colton, C. W., \& Manderscheid, R. W. (2006). Congruencies in increased mortality rates, years of potential life lost, and causes of death among public mental health clients in eight states. Preventing Chronic Disease, 3(2), 1-14.

Cook, J.A. (2006). Employment barriers for persons with psychiatric disabilities: Update of report for the President's Commission. Psychiatric Services, 57(10), 1391-1405.

Coombs, T., Nicholas, A., \& Pirkis, J. (2013). A review of social inclusion measures. Australian and New Zealand Journal of Psychiatry, 47(10), 906-919.

Corbiere, M., Samson, E., Villotti, P., \& Pelletier, J. F. (2012). Strategies to fight stigma toward people with mental disorders: Perspectives from different stakeholders. The Scientific World Journal, 1-9.

Corrigan, P., Bink, A., Fokuo, J., \& Schmidt, A. (2015). The public stigma of mental illness means a difference between you and me. Psychiatry Research, 226(1), 186- 
191.

Corrigan, P., Watson, A., Byrne, P., Davis, K. (2005). Mental illness stigma: Problem of public health or social justice? Social Work, 50(4), 363-368.

Costa, L., Voronka, J., Landry, D., Reid, J., Mcfarlane, B., Reville, D., \& Church, K. (2012). "Recovering our Stories": A small act of resistance. Studies in Social Justice, 6(1), 85-101.

Daley, A. (2010). Reflections on reflexivity and critical reflection as critical research practices. Affilia, 25(1), 68-82.

Daley, S., Newton, D., Slade, M., Murray, J., \& Banerjee, S. (2013). Development of a framework for recovery in older people with mental disorder. International Journal of Geriatric Psychiatry, 28(5), 522-529.

Darling, R. B., \& Heckert, D. A. (2010). Orientations toward disability: Differences over the life course. International Journal of Disability, Development and Education, 57(2), 131-143.

Das, A. (2012). LGBTQ women and mental health "recovery". Psychiatric Rehabilitation Journal, 35(6), 474.

Davidson, L., \& Roe, D. (2007). Recovery from versus recovery in serious mental illness: One strategy for lessening confusion plaguing recovery. Journal of Mental Health, 16(4), 459-470.

Davidson, L., Schmutte, T., Dinzeo, T., \& Andres-Hyman, R. (2008). Remission and recovery in schizophrenia: practitioner and patient perspectives. Schizophrenia Bulletin, 34(1), 5-8. 
Davis, B.A., Townley, G., \& Kloos, B. (2013). The roles of clinical and non-clinical dimensions of recovery in promoting community activities for individuals with psychiatric disabilities. Psychiatric Rehabilitation Journal, 36(1), 51-53.

Davis, F. \& Burns, J. (2015). The development and properties of the Support Needs Questionnaire. Social Inclusion, 3(4), 63-75.

Deal, M. (2003). Disabled people's attitudes toward other impairment groups: A hierarchy of impairments. Disability \& Society, 18(7), 897-910.

DeCarlo, L. T. (1997). On the meaning and use of kurtosis. Psychological Methods, 2(3), $292-307$.

De Silva, M. J., McKenzie, K., Harpham, T., \& Huttly, S. R. (2005). Social capital and mental illness: A systematic review. Journal of Epidemiology and Community Health, 59(8), 619-627.

Deutsch, A. (1948). The shame of the states. New York, NY: Harcourt, Brace, and Company.

DeVellis, R. F. (2012). Scale development: Theory and applications ( $3^{\text {rd }}$ ed.). Los Angeles, California: Sage Publications.

Dijkers, M. (2000). The Community Integration Questionnaire. The Center for Outcome Measurement in Brain Injury. http://www.tbims.org/combi/ciq.

Dillard, J., Dujon, V., \& King, M. (2009). Introduction. In J. Dillard, V. Dujon, \& King, M. (Eds.), Understanding the social dimension of sustainability (pp. 1-12). New York: Routledge.

Dillard, J., Dujon, V., \& King, M. (2009). Reflection and Directions for the Future. In J. 
Dillard, V. Dujon, \& King, M. (Eds.), Understanding the social dimension of sustainability (pp. 285-290). New York: Routledge.

Drapalski, A. L., Medoff, D., Unick, G. J., Velligan, D. I., Dixon, L. B., \& Bellack, A. S. (2012). Assessing recovery of people with serious mental illness: Development of a new scale. Psychiatric Services, 63(1), 48-53.

Duff, C. (2012). Exploring the role of "enabling places" in promoting recovery from mental illness: A qualitative test of a relational model. Health \& Place, 18(6), 13881395.

Duffy, M. E. (2006). Handling missing data: A commonly encountered problem in quantitative research. Clinical Nurse Specialist, 20(6), 273-276.

Dunkley, J. E., \& Bates, G. W. (2015). Recovery and adaptation after first-episode psychosis: The relevance of posttraumatic growth. Psychosis, 7(2), 130-140.

Dupré, M. (2012). Disability culture and cultural competency in social work. Social Work Education, 31(2), 168-183.

Durham, T. W. (1982). Norms, reliability, and item analysis of the Hopelessness Scale in general psychiatric, forensic psychiatric, and college populations. Journal of Clinical Psychology, 38(3), 597-600.

Durham, M. (1989). The impact of deinstitutionalization on the current treatment of the mentally ill. International Journal of Law and Psychiatry, 12, 117-131.

Dyce, J. A. (1996). Factor structure of the Beck Hopelessness Scale. Journal of Clinical Psychology, 52(5), 555-558.

Eaton, W. W. (1986). The sociology of mental disorders. New York: Praeger. 
Eaton, W.W., Muntaner, C., Smith, C., Tien, A., Ybarra, M. Center for Epidemiologic Studies Depression Scale: Review and revision (CESD and CESD-R). (2004). In M.E. Maruish (Ed). The use of psychological testing for treatment planning and outcomes assessment. (pp. 363-377). Mahwah, NJ: Lawrence.

Eisen, S. (1996). Behavior and Symptom Identification Scale (BASIS-32). In L.I. Sederer B. Dickey. (Eds.). Outcome assessment in clinical practice. (pp. 23-35). New York: Routledge.

Eisenberg, L. (1988). The social construction of mental illness. Psychological Medicine, $18,1-9$.

Etherington, K. (2004). Research methods: Reflexivities-roots, meanings, dilemmas. Counselling \& Psychotheraphy Research, 4(2), 46-47.

Ennis, L., \& Wykes, T. (2013). Impact of patient involvement in mental health research: Longitudinal study. The British Journal of Psychiatry, 203(5), 381-386.

Farone, D. W. (2006). Schizophrenia, community integration, and recovery: Implications for social work practice. Social Work in Mental Health, 4(4), 21-36.

Farran, C. J., Herth, K. A., \& Popovich, J. M. (1995). Hope and hopelessness: Critical clinical constructs. Washington, DC: Sage Publications, Inc.

Fidell, L. S., \& Tabachnick, B. G. (2006). Using multivariate statistics. New York: Harper and Row.

Forrest, R. (2014). The implications of adopting a human rights approach to recovery in practice: Of the various interpretations of the recovery model. Mental Health Practice,17(8), 29-33. 
Frankham, J., \& Tracy, F. (2012). Troubling the field of service user involvement in research. Contemporary Social Science, 7(1), 73-89.

Freeman, D., Startup, H., Dunn, G., Wingham, G., Černis, E., Evans, N., ... \& Kingdon, D. (2014). Persecutory delusions and psychological well-being. Social Psychiatry and Psychiatric Epidemiology, 49(7), 1045-1050.

Frese, F. J., Knight, E. L., \& Saks, E. (2009). Recovery from schizophrenia: With views of psychiatrists, psychologists, and others diagnosed with this disorder. Schizophrenia Bulletin, 35(2), 370-380.

Gabel, S., \& Peters, S. (2004). Presage of a paradigm shift? Beyond the social model of disability toward resistance theories of disability. Disability \& Society,19(6), 585-600.

Ganju, V. (1999). The MHSIP Consumer Survey. Arlington, Virginia: National Association of State Mental Health Program Directors Research Institute.

General Accounting Office (2000 December). Mental health: Community-based care increases for people with serious mental illness. United States General Accounting Office Report to the Committee on Finance, U.S. Senate.

Gillham, J. E., \& Seligman, M. E. (1999). Footsteps on the road to a positive psychology. Behaviour Research and Therapy, 37, S163-S173.

Ginsburg, F., \& Rapp, R. (2013). Disability Worlds. Annual Review of Anthropology, 42, 53-68.

Girolamo, G. D. (1996). WHO studies on schizophrenia: An overview of the results and their implications for the understanding of the disorder. The Psychotherapy Patient, 9(3-4), 213-231. 
Glaser, B. G., \& Strauss, A. L. (2009). The discovery of grounded theory: Strategies for qualitative research. Edison, New Jersey: Transaction Publishers.

Goldman, H. H., \& Grob, G. N. (2006). Defining “mental illness” in mental health policy. Health Affairs, 25(3), 737-749.

Gould, N. (2006). Social inclusion as an agenda for mental health social work. Journal of Policy Practice, 5(2-3),77-90.

Gordon, S. E. (2013). Recovery constructs and the continued debate that limits consumer recovery. Psychiatric Services, 64(3), 270-271.

Gordon, S. E., Ellis, P. M., Siegert, R. J., \& Walkey, F. H. (2013). Development of a selfassessed consumer recovery outcome measure: My voice, my life. Administration and Policy in Mental Health and Mental Health Services Research, 40(3), 199-210.

Gordon, D., Levitas, R., Pantazis, C., Patsios, D., Payne, S., Townsend, P... Williams, J. The Poverty and Social Exclusion Survey of Britain Questionnaire. Available from: https://www.jrf.org.uk/sites/default/files/jrf/migrated/files/185935128x.pdf.

Grealish, A., Tai, S., Hunter, A., \& Morrison, A. P. (2013). Qualitative exploration of empowerment from the perspective of young people with psychosis. Clinical Psychology \& Psychotherapy, 20(2), 136-148.

Green, G. (2009). The end of stigma? Changes in the social experience of long-term illness. New York: Routledge.

Griffiths, K. M., Jorm, A. F., \& Christensen, H. (2004). Academic consumer researchers: A bridge between consumers and researchers. Australian and New Zealand Journal of Psychiatry, 38(4), 191-196. 
Grob, G. (1983). Mental illness and American society, 1875-1940. New Jersey, NJ: Princeton University Press.

Grob, G. (1985). The transformation of the mental hospital in the United States. American Behavioral Scientist, 28(5), 639-653.

Grob, G. (1994). Government and mental health policy: A structural analysis. The Milbank Quarterly, 72 (3), 471-500.

Hahn, H. D., \& Belt, T. L. (2004). Disability identity and attitudes toward cure in a sample of disabled activists. Journal of Health and Social Behavior, 45(4), 453-464.

Hamano, T., Fujisawa, Y., Ishida, Y., Subramanian, S. V., Kawachi, I., \& Shiwaku, K. (2010). Social capital and mental health in Japan: A multilevel analysis. PLoS One, 5(10), e13214.

Hamer, H. P., Finlayson, M., \& Warren, H. (2014). Insiders or outsiders? Mental health service users' journeys towards full citizenship. International Journal of Mental Health Nursing, 23(3), 203-211.

Hamm, J. A., Hasson-Ohayon, I., Kukla, M., \& Lysaker, P. H. (2013). Individual psychotherapy for schizophrenia: Trends and developments in the wake of the recovery movement. Psychology Research and Behavior Management, 6, 45-54.

Hammond, C., \& Churchman, D. (2008). Sustaining academic life: A case for applying principles of social sustainability to the academic profession. International Journal of Sustainability in Higher Education, 9(3), 235-245.

Hancock, N., Bundy, A., Honey, A., Helich, S., \& Tamsett, S. (2013). Measuring the later stages of the recovery journey: Insights gained from clubhouse 
members. Community Mental Health Journal, 49(3), 323-330.

Hancock, N., Bundy, A., Tamsett, S., \& McMahon, M. (2012). Participation of mental health consumers in research: Training addressed and reliability assessed. Australian Occupational Therapy Journal, 59(3), 218-224.

Happell, B., \& Roper, C. (2007). Consumer participation in mental health research: Articulating a model to guide practice. Australasian Psychiatry, 15(3), 237-241.

Hardin, P., Padron, J., \& Manderscheid, R. (2014). White paper: US leadership and workforce development. Washington, DC: National Association of County Behavioral Health.

Harpur, P. (2012). From disability to ability: changing the phrasing of the debate. Disability \& Society, 27(3), 325-337.

Harris, M., Gladman, B., Hennessy, N., Lloyd, C., Mowry, B., \& Waghorn, G. (2011). The reliability of an education-related self-efficacy scale for people with psychiatric disabilities. Community Mental Health Journal, 47(2), 136-142.

Harris, M., Gladman, B., Hennessy, N., Lloyd, C., Mowry, B., \& Waghorn, G. (2011). Reliability and validity of a measure of role functioning among people with psychiatric disabilities. Australian Occupational Therapy Journal, 58(3), 203-208.

Hiranandani, V. (2005). Towards a critical theory of disability in social work. Critical Social Work, 6(1).

Hodgekins, J. (2012). Investigating the psychological typology of social recovery in individuals with first episode psychosis. (Doctoral dissertation). Retrieved from the University of East Anglia. 
Hodgekins, J., Birchwood, M., Christopher, R., Marshall, M., Coker, S., Everard, L.,... \& Sharma, V. (2015). Investigating trajectories of social recovery in individuals with first- episode psychosis: a latent class growth analysis. The British Journal of Psychiatry, 207(6), 536-543.

Hogan, T. P., \& Awad, A. G. (2000). Drug Attitude Inventory. Rush AJ. Handbook of psychiatric measures. Arlington, VA: American Psychiatric Association.

Hopper, K. (1991). Some old questions for the new cross-cultural psychiatry. Medical Anthropological Quarterly, 5(4), 299-330.

Hopper, K. \& Wanderling, J. (2000). Revisiting the developed versus developing country distinction in course and outcome in schizophrenia: Results from ISoS, the WHO collaborative follow up project. Schizophrenia Bulletin, 26(4), 835-846.

Hopper, K. (2007). Rethinking social recovery in schizophrenia: What a capabilities approach might offer. Social Science \& Medicine, 65(5), 868-879.

Hu, L. \& Bentler, P. (1999). Cutoff criteria for fit indexes in covariance structure analysis: Conventional criteria versus new alternatives. Structural Equation Modeling: A Multidisciplinary Journal, 6(1), 1-55.

Hughes, B. (2007). Being disabled: Towards a critical social ontology for disability studies. Disability \& Society, 22(7), 673-684.

Humphries, T. (2014). Our time: The legacy of the Twentieth Century. Sign Language Studies, 15(1), 57-73.

Ida, D. J. (2007). Cultural competency and recovery within diverse populations. Psychiatric Rehabilitation Journal, 31(1), 49. 
Jääskeläinen, E., Juola, P., Hirvonen, N., McGrath, J. J., Saha, S., Isohanni, M., Veijola, J., \& Miettunen, J. (2012). A systematic review and meta-analysis of recovery in schizophrenia. Schizophrenia Bulletin, 32(2), 1296-1306.

Jaeger, J., Berns, S. M., \& Czobor, P. (2003). The Multidimensional Scale of Independent Functioning. Schizophrenia Bulletin, 29(1), 153-168.

Jaeger, M., \& Hoff, P. (2012). Recovery: Conceptual and ethical aspects. Current Opinion in Psychiatry, 25(6), 497-502.

Jerrell, J. M. (2006). Psychometrics of the MHSIP adult consumer survey. The Journal of Behavioral Health Services \& Research, 33(4), 483-488.

Johnson, D. P., Penn, D. L., Fredrickson, B. L., Kring, A. M., Meyer, P. S., Catalino, L. I., \& Brantley, M. (2011). A pilot study of loving-kindness meditation for the negative symptoms of schizophrenia. Schizophrenia Research, 129(2), 137-140.

Jones, N., \& Brown, R. (2012). The absence of psychiatric C/S/X perspectives in academic discourse: Consequences and implications. Disability Studies Quarterly, 33(1).

Jones, N. \& Corrigan, P. (2014). Understanding stigma. In Corrigan, P. (Eds.), The stigma of disease and disability (pp.1-23). Washington, DC: APA Press.

Jones, N. \& Kelly, T. (2014). Inconvenient complications: On the heterogeneities of madness and their relationship to disability. In Spandler, H., Sapey, B., \& Anderson, J. (Eds.), Madness and the politics of disablement (pp. 1-16). London: Polity Press. Jordan, I. K. (2005). The Gallaudet experience: Deafness and disability. PMLA, 120 (2), 625-627. 
Joseph, A. J. (2013). Empowering alliances in pursuit of social justice: Social workers supporting psychiatric-survivor movements. Journal of Progressive Human Services, 24(3), 265-288.

Judge, A., Estroff, S., Perkins, D., \& Penn, D. (2008). Recognizing and responding to early psychosis: A qualitative analysis of individual narratives. Psychiatric Services, 59(1), 96-99.

Kao, Y. C., Liu, Y. P., \& Lu, C. W. (2012). Beck Hopelessness Scale: Exploring its dimensionality in patients with schizophrenia. Psychiatric Quarterly, 83(2), 241-255.

Kaplan, K., Salzer, M. S., \& Brusilovskiy, E. (2012). Community participation as a predictor of recovery-oriented outcomes among emerging and mature adults with mental illnesses. Psychiatric Rehabilitation Journal, 35(3), 219-229.

Kara, H. (2013). Mental health service user involvement in research: Where have we come from, where are we going? Journal of Public Mental Health, 12(3), 122-135.

Karon, B. P., \& Widener, A. J. (1999). The tragedy of schizophrenia: Its myth of incurability. Ethical Human Sciences and Services, 1(3), 195-211.

Kay, S. R., Flszbein, A., \& Opfer, L. A. (1987). The positive and negative syndrome scale (PANSS) for schizophrenia. Schizophrenia Bulletin, 13(2), 261.

Kemp, D. R. (2007). Mental health in America: A reference handbook. Oxford, England: ABC-CLIO.

Kiejna, A., Rymaszewska, J., Hadryś, T., Suwalska, A., Łojko, D., \& Rybakowski, J. K. (2006). Bipolar or unipolar? - the question for clinicians and researchers. Journal of Affective Disorders, 93(1), 177-183. 
Killaspy, H., White, S., Lalvani, N., Berg, R., Thachil, A., Kallumpuram, S., Nasiruddin, O., Wright, C., \& Mezey, G. (2013). The impact of psychosis on social inclusion and associated factors. International Journal of Social Psychiatry, 602,148-154.

Kline, R. (2011). Principles and practice of structural equation modeling ( $3^{\text {rd }}$ edition). New York: Guilford Press.

Knapp, Martin and Chisholm, D. (2006) Introduction and manual for the CSSRI-

EU. In G. Thornicroft, T. Becker, M. Knapp, H. Knudsen, C. Helle, A. Schene, M. Tansella, and J. Vazquez-Barquero. (Eds.) International outcome measures in mental health: Quality of life, needs, service satisfaction, costs and impact on carers. (pp. 7175). London: Gaskell.

Kudlick, C. J. (2003). Disability history: Why we need another "other". The American Historical Review, 108(3), 763-793.

Laing, R.D. (1960). The divided self: An existential study in sanity and madness. New York: Penguin.

Lamers, S. M., Westerhof, G. J., Glas, C. A., \& Bohlmeijer, E. T. (2015). The bidirectional relation between positive mental health and psychopathology in a longitudinal representative panel study. The Journal of Positive Psychology, 10 (6), $553-560$.

Lampropoulos, G. K. (2001). Integrating psychopathology, positive psychology, and psychotherapy. American Psychologist, 56(1), 87-88

Law, H., Morrison, A., Byrne, R., \& Hodson, E. (2012). Recovery from psychosis: A user informed review of self-report instruments for measuring recovery. Journal of 
Mental Health, 21(2), 192-207.

Law, H., Neil, S. T., Dunn, G., \& Morrison, A. P. (2014). Psychometric properties of the Questionnaire about the Process of Recovery (QPR).Schizophrenia Research (156), 184-189.

Leamy, M., Bird, V., Le Boutillier, C., Williams, J., \& Slade, M. (2011). Conceptual framework for personal recovery in mental health: Systematic review and narrative synthesis. The British Journal of Psychiatry, 199(6), 445-452.

Le Boutillier, C. L., \& Croucher, A. (2010). Social inclusion and mental health. The British Journal of Occupational Therapy, 73(3), 136-139.

Lê Cook, B., Carson, N., \& Alegria, M. (2010). Assessing racial/ethnic differences in the social consequences of early-onset psychiatric disorder. Journal of Health Care for the Poor and Underserved, 21(2 Suppl), $49-66$.

Lencucha, R., Kinsella, E. A., \& Sumsion, T. (2008). The formation and maintenance of social relationships among individuals living with schizophrenia. American Journal of Psychiatric Rehabilitation, 11(4), 330-355.

Liberman, R. P., \& Kopelowicz, A. (2005). Recovery from schizophrenia: A concept in search of research. Psychiatric Services, 56(6), 735-742.

Lincoln, Y. S. (1985). Naturalistic inquiry. Newbury Park, CA: Sage.

Lindahl, K. , Balajee, S., \& Wiggins, N. (2013). Complete health equity: Bringing mental health into the equity dialogue. In Veronica Dujon, Jesse Dillard, \& Eileen Brennan (Eds)., Social sustainability: A multilevel approach to social inclusion (pp.158-182). New York: Routledge. 
Little, R. J., \& Rubin, D. B. (2002). Statistical analysis with missing data. New York: Wiley.

Livneh, H. \& Martz, E. (2015). Psychosocial adaptation to disability within the context of positive psychology: Findings from the literature. Journal of Occupational Rehabilitation, 1-9.

Lloyd, C., Waghorn, G., Best, M., \& Gemmell, S. (2008). Reliability of a composite measure of social inclusion for people with psychiatric disabilities. Australian Occupational Therapy Journal, 55(1), 47-56.

Loja, E., Costa, M. E., Hughes, B., \& Menezes, I. (2013). Disability, embodiment and ableism: Stories of resistance. Disability \& Society, 28(2), 190-203.

Luchins, A. (2001). Moral treatment in asylums and general hospitals in nineteenth century America. The Journal of Psychology, 12(3), 585-607.

Lysaker, P. H., Ringer, J., Maxwell, C., McGuire, A., \& Lecomte, T. (2010). Personal narratives and recovery from schizophrenia. Schizophrenia Research,121(1), 271-276.

Lysaker, P. H., \& Silverstein, S. M. (2009). Psychotherapy of schizophrenia: A brief history and the potential to promote recovery. Clinical Case Studies, 8(6), 417-423.

McRuer, R. (2011). Disabling sex: Notes for a crip theory of sexuality. GLQ: A Journal of Lesbian and Gay Studies, 17(1), 107-117.

Malmberg-Heimonen, I. (2009). The social capital and mental health of long-term social assistance recipients in Norway. European Journal of Social Work, 13(1), 91-107.

Mancini, M. A. (2007). A qualitative analysis of turning points in the recovery process. American Journal of Psychiatric Rehabilitation, 10(3), 223-244. 
Mancini, M. A., Hardiman, E. R., \& Lawson, H. A. (2005). Making sense of it all:

Consumer providers' theories about factors facilitating and impeding recovery from psychiatric disabilities. Psychiatric Rehabilitation Journal, 29(1), 48-55.

Manderscheid, R. W., Ryff, C. D., Freeman, E. J., McKnight-Eily, L. R., Dhingra, S., \& Strine, T. W. (2010). Evolving definitions of mental illness and wellness. Preventing Chronic Disease, 7(1), 1-6.

Marino, C.K. (2015). To belong, contribute, and hope: First stage development of a measure of social recovery. Journal of Mental Health, 24(2), 68-72.

Marino-Francis, F., \& Worrall-Davies, A. (2010). Development and validation of a social inclusion questionnaire to evaluate the impact of attending a modernized mental health day service. Mental Health Review Journal, 15(1), 37-48.

Mezey, Gillian, Sarah White, Ajoy Thachil, Rachel Berg, Sen Kallumparam, Omar Nasiruddin, Christine Wright, and Helen Killaspy. (2013). Development and preliminary validation of a measure of social inclusion for use in people with mental health problems: The SInQUE." International Journal of Social Psychiatry 59 (5), $501-507$.

Mezzina, R., Davidson, L., Borg, M., Marin, I., Topor, A., \& Sells, D. (2006). The social nature of recovery: Discussion and implications for practice. American Journal of Psychiatric Rehabilitation, 9(1), 63-80.

Meyer, P. S., Johnson, D. P., Parks, A., Iwanski, C., \& Penn, D. L. (2012). Positive living: A pilot study of group positive psychotherapy for people with schizophrenia. The Journal of Positive Psychology, 7(3), 239-248. 
Miller, D. \& Blanc, E. (1967). Concepts of "Moral Treatment" for the mentally ill: Implications for social work with post hospital mental patients. Social Service Review, 41(1), 66-74.

Mohr, P., Rodriguez, M., Bravermanová, A., Melicher, T., Čeplová, Z., Čermák, J., \& Pečeňák, J. (2013). Social and functional capacity of schizophrenia patients: A crosssectional study. International Journal of Social Psychiatry, 6094), 352-360.

Moltu, C., Stefansen, J., Svisdahl, M., \& Veseth, M. (2013). How to enhance the quality of mental health research: Service users' experiences of their potential contributions through collaborative methods. American Journal of Psychiatric Rehabilitation, 16(1), $1-21$.

Monger, B., Hardie, s., Ion, R., Cumming, J., \& Henderson, N. (2013). The Individual Recovery Outcomes Counter: Preliminary validation of a personal recovery measure. The Psychiatrist, 37, 221-227.

Moran, G. S., \& Nemec, P. B. (2013). Walking on the sunny side: what positive psychology can contribute to psychiatric rehabilitation concepts and practice. Psychiatric Rehabilitation Journal, 36(3), 202-208.

Morgan, C., Burns, T., Fitzpatrick, R., Pinfold, V., \& Priebe, S. (2007). Social exclusion and mental health: Conceptual and methodological review. The British Journal of Psychiatry, 191(6), 477-483.

Morrissey, J. \& Goldman, H. (1986). Care and treatment of the mentally ill in the United States: Historical developments and reform. The Annals of the American Academy of Political and Social Science, 484, 12-27. 
Morrow, M., \& Weisser, J. (2012). Towards a social justice framework of mental health recovery. Studies in Social Justice, 6(1), 27-43.

Mulvany, J. (2000). Disability, impairment or illness? The relevance of the social model of disability to the study of mental disorder. Sociology of Health \& Illness,22(5), 582 601.

Munro, B. H. (2005). Statistical methods for health care research ( $5^{\text {th }}$ ed.) Philadelphia, PA: Lippincott Williams \& Wilkins.

Nash, M. (2002). Voting as a means of social inclusion for people with a mental illness. Journal of Psychiatric and Mental Health Nursing, 9(6), 697-703.

National Association of Social Workers. (2015). Social Work speaks: National Association of Social Workers policy statements. Washington, DC: NASW Press.

National Association of State Mental Health Program Directors (NASMHPD). (2007). Promoting independence and recovery through work: Employment for people with psychiatric disabilities. Washington, DC: National Governors Association. National Institute of Mental Health (NIMH). Serious mental health illness (SMI) among U.S. adults. Retrieved from: http://nimh.nih.gov/health/statistics/prevalence/serious-mental-illnessNeil, S. T., Kilbride, M., Pitt, L., Nothard, S., Welford, M., Sellwood, W., \& Morrison, A.(2009). The questionnaire about the process of recovery (QPR): A measurement tool developed in collaboration with service users. Psychosis, 1(2), 145-155.

New Freedom Commission on Mental Health. (2003). Achieving the promise: Transforming mental healthcare in America. Final report. DHHS Pub No. SMA-03- 
3832. Rockville, MD.

Norman, R. M., Windell, D., Lynch, J., \& Manchanda, R. (2013). Correlates of subjective recovery in an early intervention program for psychoses. Early Intervention in Psychiatry, 7(3), 278-284.

Nunnally, J. (1978). Psychometric theory (2 ${ }^{\text {nd }}$ edition). New York: McGraw-Hill.

Ochoa, S., Usall, J., Cobo, J., Labad, X., \& Kulkarni, J. (2012). Gender differences in schizophrenia and first-episode psychosis: A comprehensive literature review. Schizophrenia Research and Treatment, 1-9.

Olin, E., Nordström, M., \& Wijk, H. (2011). Privacy, meetings and rejections-a qualitative study of everyday life among young persons with psychiatric disabilities. Scandinavian Journal of Disability Research, 13(2), 135-150.

Onken, S. J., Craig, C. M., Ridgway, P., Ralph, R. O., \& Cook, J. A. (2007). An analysis of the definitions and elements of recovery: A review of the literature. Psychiatric Rehabilitation Journal, 31(1), 9.

Or, S., Hasson-Ohayon, I., Feingold, D., Vahab, K., Amiaz, R., Weiser, M., \& Lysaker, P. H. (2013). Meaning in life, insight and self-stigma among people with severe mental illness. Comprehensive psychiatry, 54(2), 195-200.

Ostrow, L., \& Adams, N. (2012). Recovery in the USA: From politics to peer support. International Review of Psychiatry, 24(1), 70-78.

Padden, C. A. (2005). Talking culture: Deaf people and disability studies. PMLA, 120(2), 508-513.

Pallant, J. (2010). SPSS survival manual: A step by step guide to data analysis using 
SPSS. New York: McGraw-Hill International.

Palmer, B. W., Martin, A. S., Depp, C. A., Glorioso, D. K., \& Jeste, D. V. (2014).

Wellness within illness: Happiness in schizophrenia. Schizophrenia Research, 159(1), $151-156$.

Patterson, S., Trite, J., \& Weaver, T. (2014). Activity and views of service users involved in mental health research: UK survey. The British Journal of Psychiatry,

Payne, C. (2009). Asylum: Inside the closed world of the state mental hospitals. Cambridge, MA: MIT Press.

Perry, B. M., Taylor, D., \& Shaw, S. K. (2007). "You've got to have a positive state of mind": An interpretative phenomenological analysis of hope and first episode psychosis. Journal of Mental Health, 16(6), 781-793.

Peters, S., Gabel, S., \& Symeonidou, S. (2009). Resistance, transformation and the politics of hope: Imagining a way forward for the disabled people's movement. Disability \& Society, 24(5), 543-556.

Peterson, C. (2009). Positive psychology. Reclaiming Children and Youth, 18(2), 3 -11.

Phelan, M., Slade, M., Thornicroft, G., Dunn, G., Holloway, F., Wykes, T., ... \& Hayward, P. (1995). The Camberwell Assessment of Need: The validity and reliability of an instrument to assess the needs of people with severe mental illness. The British Journal of Psychiatry, 167(5), 589-595.

Phillips, R. (2006). Consumer participation in mental health research. Social Policy Journal of New Zealand, 27, $171-182$.

Pilling, M. D. (2012). Invisible identity in the workplace: Intersectional madness and 
processes of disclosure at work. Disability Studies Quarterly, 33(1).

Pitt, L., Kilbride, M., Nothard, S., Welford, M., \& Morrison, A. P. (2007). Researching recovery from psychosis: A user-led project. Psychiatric Bulletin, 31(2), 55-60.

Priebe, S. (2007). Social outcomes in schizophrenia. The British Journal of Psychiatry, 191(50), s15-s20.

Ralph, R. O. (2002). The dynamics of disclosure: Its impact on recovery and rehabilitation. Psychiatric Rehabilitation Journal, 26(2), 165-172.

Ramon, S., Healy, B., \& Renouf, N. (2007). Recovery from mental illness as an emergent concept and practice in Australia and the UK. International Journal of Social Psychiatry, 53(2), 108-122.

Rapp, R., \& Ginsburg, F. (2012). Disability worlds. Annual Review of Anthropology, 42(4), 15-33.

Resnick, S. G., \& Rosenheck, R. A. (2006). Recovery and positive psychology: Parallel themes and potential synergies. Psychiatric Services, 57(1), 120-122.

Resnick, S. G., Rosenheck, R. A., \& Lehman, A. F. (2004). An exploratory analysis of correlates of recovery. Psychiatric Services, 55(5), 540-547.

Ridgway, P. (2001). Restorying psychiatric disability: Learning from first person recovery narratives. Psychiatric Rehabilitation Journal, 24(4), 335.

Roe, D., Mashiach-Eizenberg, M., \& Lysaker, P. (2011). The relation between objective and subjective domains of recovery among persons with schizophrenia-related disorders. Schizophrenia Research, 131, 133-138.

Rogers, E. S., Teague, G. B., Lichenstein, C., Campbell, J., Lyass, A., Chen, R., \& 
Banks, S. (2007). Effects of participation in consumer-operated service programs on both personal and organizationally mediated empowerment: results of multisite study. Journal of Rehabilitation Research and Development 44(6), 785-800.

Romano, D., McCay, E., Goering, P., Boydell, K., \& Zipursky, R. (2010). Reshaping an enduring sense of self: The process of recovery from a first episode of schizophrenia. Early Intervention in Psychiatry, 4, 243-250.

Rosa, A. R., Sánchez-Moreno, J., Martínez-Aran, A., Salamero, M., Torrent, C., Reinares, M. Comes, M., Colom, F., Van Riel, W., Ayuso-Mateios, Kapczinski, F., \& Vieta, E. (2007). Validity and reliability of the Functioning Assessment Short Test (FAST) in bipolar disorder. Clinical Practice and Epidemiology in Mental Health, 3(1), 1-6.

Rose, V. K., \& Thompson, L. M. (2012). Space, place and people: A community development approach to mental health promotion in a disadvantaged community. Community Development Journal, 47(4), 604-611.

Rothman, D. (1980). Conscience and convenience: The asylum and its alternatives in progressive America. Boston, MA: Little Brown \& Company.

Rüsch, N., Abbruzzese, E., Hagedorn, E., Hartenhauer, D., Kaufmann, I., Curschellas, J., ... \& Corrigan, P. W. (2014). Efficacy of Coming Out Proud to reduce stigma's impact among people with mental illness: Pilot randomized controlled trial. The British Journal of Psychiatry, 204(5), 391-397.

Rüsch, N., Nordt, C., Kawohl, W., Brantschen, E., Bärtsch, B., Müller, M., Corrigan, P., \& Rössler, W. (2014). Work-related discrimination and change in self-stigma among 
people with mental illness during supported employment. Psychiatric Services, 65(12), 1496-1498.

Rymaszewska, J., Jarosz-Nowak, J., Kiejna, A., Kallert, T., Schützwohl, M., Priebe, S., Wright, D., Nawka, P., \& Raboch, J. (2007). Social disability in different mental disorders. European Psychiatry, 22(3), 160-166.

Salzer, M. S., \& Brusilovskiy, E. (2014). Advancing recovery science: Reliability and validity properties of the Recovery Assessment Scale. Psychiatric Services, 65(4), $442-453$.

Salzer, M. S., Brusilovskiy, E., Prvu-Bettger, J., \& Kottsieper, P. (2014). Measuring community participation of adults with psychiatric disabilities: Reliability of two modes of data collection. Rehabilitation Psychology, 59(2), 211.

SAMHSA (2004). National consensus statement on mental health recovery. Rockville, Maryland: U.S. Department of Health and Human Services.

Satcher, D. (2000). Mental health: A report of the Surgeon General--Executive summary. Professional Psychology: Research and Practice, 31(1), 5-13.

Schon, U. K. (2009). How men and women in recovery give meaning to severe mental illness. Journal of Mental Health, 18(5), 433-440.

Schön, U. K., Denhov, A., \& Topor, A. (2009). Social relationships as a decisive factor in recovering from severe mental illness. The International Journal of Social Psychiatry, 55(4), 336-347.

Schrader, S., Jones, N., \& Shattell, M. (2013). Mad pride: Reflections on sociopolitical identity and mental diversity in the context of culturally competent psychiatric 
care. Issues in Mental Health Nursing, 34(1), 62-64.

Schrank, B., Bird, V., Rudnick, A., \& Slade, M. (2012). Determinants, self-management strategies and interventions for hope in people with mental disorders: Systematic search and narrative review. Social Science \& Medicine, 74(4), 554-564.

Schrank, B., Brownell, T., Tylee, A., \& Slade, M. (2014). Positive psychology: An approach to supporting recovery in mental illness. East Asian Archives of Psychiatry, 24(3), 95-103.

Schrank, B., Woppmann, A., Grant Hay, A., Sibitz, I., Zehetmayer, S., \& Lauber, C. (2012). Validation of the Integrative Hope Scale in people with psychosis. Psychiatry Research, 198(3), 395-399.

Secker, J., Hacking, S., Kent, L., Shenton, J., \& Spandler, H. (2009). Development of a measure of social inclusion for arts and mental health project participants. Journal of Mental Health, 18(1), 65-72.

Secker, J., Membrey, H., Grove, B., \& Seebohm, P. (2002). Recovering from illness or recovering your life? Implications of clinical versus social models of recovery from mental health problems for employment support services. Disability \& Society, 17(4), 403-418.

Seligman, M. E., \& Csikszentmihalyi, M. (2000). Positive psychology: An introduction. American Psychologist, 55(1), 5-14.

Senghas, R. J., \& Monaghan, L. (2002). Signs of their times: Deaf communities and the culture of language. Annual Review of Anthropology, 31, 69-97.

Shakespeare, T. (2008). Disability: suffering, social oppression, or complex predicament? 
International Library of Ethics, Law \& the New Medicine, 39, 235-246.

Shanks, V., Williams, J., Leamy, M., Bird, V. J., Le Boutillier, C., \& Slade, M. (2013). Measures of personal recovery: A systematic review. Psychiatric Services, 64(10), 974-980.

Shepherd, S., Depp, C. A., Harris, G., Halpain, M., Palinkas, L. A., \& Jeste, D. V. (2012). Perspectives on schizophrenia over the lifespan: A qualitative study. Schizophrenia Bulletin, 38(2), 295-303.

Sherry, M. (2004). Overlaps and contradictions between queer theory and disability studies. Disability \& Society, 19(7), 769-783.

Shippee, N. D., Domecq Garces, J. P., Prutsky Lopez, G. J., Wang, Z., Elraiyah, T. A., Nabhan, M., Brito, J., Boehmer, K., Hasen, R., Firwana, B., Erwin, P., Montori, V. \& Murad, M. H. (2015). Patient and service user engagement in research: A systematic review and synthesized framework. Health Expectations, 18(5), 1151-1166.

Singh, A. N. (1999). Shamans, healing, and mental health. Journal of Child and Family Studies, 8(2), 131-134.

Skultans, V. (1975). Madness and morals: Ideas on insanity in the nineteenth century. Boston, MA: Routledge \& Kegan Paul.

Slade, M. (2010). Mental illness and well-being: The central importance of positive psychology and recovery approaches. British Medical Journal Health Services Research, 10(26), 1-14.

Slade, M., Amering, M., Farkas, M., Hamilton, B., O'Hagan, M., Panther, G., Perkins, R., Shepherd, G., Tse, S., \& Whitley, R. (2014). Uses and abuses of recovery: 
Implementing recovery-oriented practices in mental health systems. World Psychiatry, 13(1), 12-20.

Slade, M., Leamy, M., Bacon, F., Janosik, M., Le Boutillier, C., Williams, J., \& Bird, V. (2012). International differences in understanding recovery: Systematic review. Epidemiology and Psychiatric Sciences, 21(04), 353-364.

Slate, R. \& Johnson, W. (2008). The criminalization of mental illness. Durham, North Carolina: Carolina Academic Press.

Smith-Merry, J., Freeman, R., \& Sturdy, S. (2011). Implementing recovery: An analysis of the key technologies in Scotland. International Journal of Mental Health Systems, 5(11), 1-12.

Springer, D. W. (1998). Validation of the Adolescent Concerns Evaluation (ACE): Detecting indicators of runaway behavior in adolescents. Social Work Research, 22(4), 241-250.

Staley, K., Kabir, T., \& Szmukler, G. (2013). Service users as collaborators in mental health research: Less stick, more carrot. Psychological Medicine, 43(06), 1121-1125.

Steed, L. (2001). Further validity and reliability evidence for Beck Hopelessness Scale scores in a nonclinical sample. Educational and Psychological Measurement, 61(2), 303-316. Stewart, G., Sara, G., Harris, M., Waghorn, G., Hall, A., Sivarajasingam, S., ... \& Mowry, B. (2010). A brief measure of vocational activity and community participation: development and reliability of the Activity and Participation Questionnaire. Australian and New Zealand Journal of Psychiatry, 44(3), 258-266. Stickley, T. \& Shaw, R. (2005). Evaluating social inclusion. Mental Health Practice, 
9(10), 14-20.

Stickley, T., \& Wright, N. (2011). The British research evidence for recovery, papers published between 2006 and 2009 (inclusive). Part One: A review of the peerreviewed literature using a systematic approach. Journal of Psychiatric and Mental Health Nursing, 18(3), 247-256.

Stickley, T., \& Wright, N. (2011). The British research evidence for recovery, papers published between 2006 and 2009 (inclusive). Part two: A review of the grey literature including book chapters and policy documents. Journal of Psychiatric and Mental Health Nursing, 18(4), 297-307.

Stienstra, D., \& Ashcroft, T. (2010). Voyaging on the seas of spirit: An ongoing journey towards understanding disability and humanity. Disability \& Society, 25(2), 191-203.

Stuart, P. (1997). Community care and the origins of psychiatric social work. Social Work in Health Care, 25(3), 25-36.

Swarbrick, M. (2009). Historical perspective: From institution to community. Occupational Therapy in Mental Health, 25(3-4), 201-223.

Szasz, T. (1974). The myth of mental illness. New York: Harper \& Row.

Telford, R., \& Faulkner, A. (2004). Learning about service user involvement in mental health research. Journal of Mental Health, 13(6), 549-559.

Tew, J. (2013). Recovery capital: what enables a sustainable recovery from mental health difficulties? European Journal of Social Work, 16(3), 360-374.

Tew, J., Ramon, S., Slade, M., Bird, V., Melton, J., \& Le Boutillier, C. (2011). Social factors and recovery from mental health difficulties: A review of the evidence. British 
Journal of Social Work, 42, 443-460.

Thomas, C. (2006). Disability and gender: Reflections on theory and research. Scandinavian Journal of Disability Research, 8(2-3), 177-185.

Thompson, K. N., McGorry, P. D., \& Harrigan, S. M. (2003). Recovery style and outcome in first-episode psychosis. Schizophrenia Research, 62(1), 31-36.

Titchkosky, T. \& Aubrecht, K. (2009). The anguish of power: Remapping mental diversity with an anticolonial compass. In A. Kempf (Ed.), Breaching the colonial contract: Anti-colonialism in the US and Canada (pp. 179-199). New York: Springer.

Tomes, N. (2006). The patient as a policy factor: A historical case study of the consumer/survivor movement in mental health. Health Affairs, 25(3), 720-729.

Tooth, B., Kalyanasundaram, V., Glover, H., \& Momenzadah, S. (2003). Factors consumers identify as important to recovery from schizophrenia. Australasian Psychiatry, 11(s1), S70-S77.

Topor, A., Borg, M., Di Girolamo, S., \& Davidson, L. (2009). Not just an individual journey: Social aspects of recovery. International Journal of Social Psychiatry, 57, 90-99.

Tyrer, P., Nur, U., Crawford, M., Karlsen, S., MacLean, C., Rao, B., \& Johnson, T. (2005). The Social Functioning Questionnaire: A rapid and robust measure of perceived functioning. International Journal of Social Psychiatry, 51(3), 265-275.

U.S. Department of Health and Human Services (1999). Mental health: A report of the Surgeon General - Executive summary. Rockville, MD: SAMHSA.

U.S. Department of Health and Human Services. National consensus statement on mental 
health recovery. Rockville, MD: SAMHSA.

U.S. Department of Justice (2007). Prison statistics: Summary findings. Retrieved from http://www.ojp.usdoj.gov /prisons.htm

Vandekinderen, C., Roets, G., Roose, R., \& Van Hove, G. (2012). Rediscovering recovery: Reconceptualizing underlying assumptions of citizenship and interrelated notions of care and support. The Scientific World Journal, 2012.

van Gestel-Timmermans, J. A. W. M., Brouwers, E. P. M., Bongers, I. L., van Assen, M. \& van Nieuwenhuizen, C. (2011). Profiles of individually defined recovery of people with major psychiatric problems. International Journal of Social Psychiatry, 58(5), $521-531$.

Ventura, J., Green, M. F., Shaner, A., \& Liberman, R. P. (1993). Training and quality assurance with the Brief Psychiatric Rating Scale: "The drift busters." International Journal of Methods in Psychiatric Research. 3, 221-224.

Vila-Rodriguez, F., Ochoa, S., Autonell, J., Usall, J., \& Haro, J. M. (2011). Complex interaction between symptoms, social factors, and gender in social functioning in a community-dwelling sample of schizophrenia. Psychiatric Quarterly, 82(4), 261-274.

Upthegrove, R., Atulomah, O., Brunet, K., \& Chawla, R. (2013). Cultural and social influences of negative illness appraisals in first-episode psychosis. Early Intervention in Psychiatry, 7(4), 399-406.

U.S. Census Bureau. (2014). Quick facts. Retrieved from: https://www.census.gov/quickfacts/table/PST045215/00.

Waghorn, G., Chant, D., \& King, R. (2005a). Work-related self-efficacy among 
community residents with psychiatric disabilities. Psychiatric Rehabilitation Journal, 29(2), 105-113.

Waghorn, G., Chant, D., \& King, R. (2005b). Work-related subjective experiences among community residents with schizophrenia or schizoaffective disorder. Australian and New Zealand Journal of Psychiatry,39(4), 288-299.

Walsh, F. P., \& Tickle, A. C. (2013). Working towards recovery: The role of employment in recovery from serious mental health problems: A qualitative metasynthesis. International Journal of Psychosocial Rehabilitation, 17 (2) 35-49.

Wang, D. (2012). The use of self and reflective practice in relational teaching and adult learning: A social work perspective. Reflective Practice, 13(1), 55-63.

Ware, N., Hopper, K., Tugenberg, T., Dickey, B., \& Fisher, D. (2007). Connectedness and citizenship: Redefining social integration. Psychiatric Services, 58(4), 469-474.

Webber, M., Corker, E., Hamilton, S., Weeks, C., Pinfold, V., Rose, D., Thornicroft, G., \& Henderson, C. (2014). Discrimination against people with severe mental illness and their access to social capital: Findings from the Viewpoint survey. Epidemiology and Psychiatric Sciences, 23(02), 155-165.

Weissman, M. M. (1999). Social Adjustment Scale-self Report (SAS-SR): User's manual. Cheektowaga, New York: Multi-Health Systems.

Whitley, R., \& Drake, R. (2010). Recovery: A dimensional approach. Psychiatric Services, 61(12), 1248-1250.

Whitley, R., \& McKenzie, K. (2005). Social capital and psychiatry: Review of the literature. Harvard Review of Psychiatry, 13(2), 71-84. 
Williams, C. C., \& Collins, A. A. (2002). The social construction of disability in schizophrenia. Qualitative Health Research, 12(3), 297-309.

Windell, D. \& Norman, R. (2012). A qualitative analysis of influences on recovery following a first episode of psychosis. International Journal of Social Psychiatry, 59(5), 493-500.

Wolbring, G., \& Rybchinski, T. (2013). Social sustainability and its indicators through a disability studies and an ability studies lens. Sustainability, 5(11), 4889-4907.

Wolframe, P. M. (2013). The Madwoman in the academy, or, Revealing the invisible straightjacket: Theorizing and teaching sanism and sane privilege. Disability Studies Quarterly, 33(1).

Wong, Y. L. I., Sands, R. G., \& Solomon, P. L. (2010). Conceptualizing community: The experience of mental health consumers. Qualitative Health Research, 20(5), 654-667.

World Psychiatric Association (2016). Stigma and mental illness. Retrieved from http://www.wpanet.org/detail.php?section_id=11\&content_id=555.

Wright, N., \& Stickley, T. (2013). Concepts of social inclusion, exclusion and mental health: A review of the international literature. Journal of Psychiatric and Mental Health Nursing, 20(1), 71-81.

Yates, I., Holmes, G., \& Priest, H. (2011). There seems no place for place: A gap analysis of the recovery literature. Journal of Public Mental Health, 10(3), 140-150.

Yilmaz, M., Josephsson, S., Danermark, B., \& Ivarsson, A. B. (2009). Social processes of participation in everyday life among persons with schizophrenia. International Journal of Qualitative Studies on Health and Well-being, 4(4), 267-279. 
Young, M. A., Halper, I. S., Clark, D. C., Scheftner, W., \& Fawcett, J. (1992). An itemresponse theory evaluation of the Beck Hopelessness Scale. Cognitive Therapy and Research, 16(5), 579-587.

Zelenev, A. (2011). Analyzing and measuring social exclusion in the United States.

(Doctoral dissertation). Retrieved from Yale University 


\section{Appendix A}

\section{IRB Approval}

\section{PS Portland State}
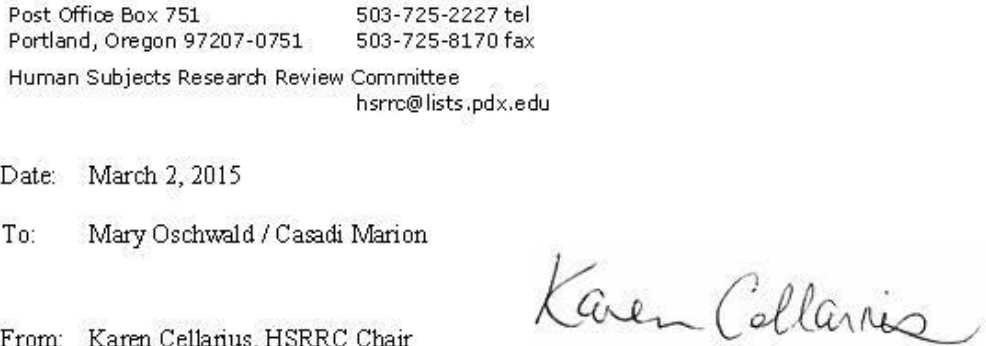

Re: HSRRC approval for your project titled, "Development of a Social Recovery Measure" HSRRC Proposal \# 153336

Approval-Expiration: March 2, 2015-March 1,2016

Review Type: Expedited, Categories 6,7

In accordance with your request, the PSU Human Subjects Research Review Committee has reviewed your request for approval of the project referenced above for compliance with PSU and DHHS policies and regulations covering the protection of human subjects. The Committee is satisfied that your provisions for protecting the rights and welfare of all subjects participating in the research are adequate, and your project is approved. Please note the following requirements:

Approval: You are approved to conduct this research study only during the period of approval cited above; and the research must be conducted according to the plans and protocol submitted (approved copy enclosed).

Consent: Signed consent is required from all participants in focus groups and interviews for this study. Signed consent is waived for survey participants. A written consent statement for the surveys is required.

Changes to Protocol: Any changes in the proposed study, whether to procedures, survey instruments, consent forms or cover letters, must be outlined and submitted to the Committee immediately. The proposed changes cannot be implemented before they have been reviewed and approved by the Committee.

Continuing Review: This ayproval will expire on 03/01/2016. It is the investigator's responsibility to ensure that a Continuing Review Report on the status of the project is submitted to the HSRRC two months before the expiration date, and that approval of the study is kept current. The $\mathbb{R B}$ offices does not send out notifications of expiration dates. The Continumg Review Report is available at www.rsp.pdx. edu/compliance_human.php and in the Office of Research and Strategic Partnerships (RSP).

Adverse Reac tions and/or Unanticipated Problems: If any adverse reactions or unanticipated problems occur as a result of this study, you are required to notify the Committee immediately. If the issue is serious, approval may be withdrawn pending an investigation by the Committee.

Completion of Study: Please notify the Committee as soon as your research has been completed. Study records, including protocols and signed consent forms for each participant, must be kept by the investigator in a secure location for three years following completion of the study (or per any requirements specified by the project's funding agency)

If you have questions or concerns, please contact the Office of Research Integrity in the PSU RSP at 503-725-2227. 


\title{
Appendix B
}

\section{Qualtrics Survey}

Welcome

I am a PhD student in Social Work at Portland State University. I am creating a measure of social recovery as part of my dissertation. My adviser Dr. Mary Oschwald is helping me. Social recovery is defined as the ability to lead a full, contributing, active life. It refers to social interaction, meaningful activity, and community participation.

The purpose of this study is to develop a measure of social recovery To do so, I need people in recovery to take the measure and three others that have already been developed to see if my measure performs as expected.

To participate, you must be 18 years or older and in recovery from a mental health challenge. Participation is voluntary. Those who take the survey can choose to enter a drawing for one of two $\$ 100$ Visa gift cards.

The computer-based survey will take about $\mathbf{4 0}$ minutes to complete. Results will be reported for the group of participants as a whole. Your name will not be used.

Thank you for your consideration. If you would like to take the survey, please continue to the next screen.

Good thoughts to you,

Casadi "Khaki" Marino, casadi@pdx.edu

Mary Oschwald, oschwald@pdx.edu

\author{
Consent to Take Part in Research \\ Portland State University \\ School of Social Work \\ Primary Investigator: Dr. Mary Oschwald \\ Co-Researcher: Casadi "Khaki" Marino
}

\section{Introduction}

- You are being asked to take part in a research study that examines the social aspects of recovery.

- This study is part of Casadi "Khaki" Marino's doctoral education at Portland State University (PSU).

-

Why have I been asked to take part in the study?

- Because you are over 18 years of age and are in recovery from mental health challenges.

- Because you might want to contribute to research on the social aspects of recovery.

-

What do I do first?

- Before agreeing, please read this form.

- Please ask any questions that you may have.

-

What is the Study about?

- The study is focused on developing a measure of social recovery.

Who will take part in the Study? 
If I agree to take part, what will I be asked to do?

- Complete the measure that is being developed and three similar measures.

- Respond to some questions about your gender, age, race/ethnicity, etc.

- 50 people will be asked to take the measure being developed again in two-weeks time to make sure the measure that is being developed measures consistently over time.

What are the risks of being in the study?

- Completing the measures may bring up memories and feelings. If you wish, providers and peers of your agency will be available to talk to you about your experiences.

What are the benefits of being in the study?

- You will contribute to the research on the social aspects of recovery.

- You can choose to enter a drawing for one of two $\$ 100$ Visa gift cards for taking the survey once.

- Those who agree to take the measure being developed again in two-weeks time can enter a drawing for a $\$ 50$ Visa gift card.

How will things I say be kept private?

- The records of this study will be kept private.

- In any type of report we may write, we will not include your name or anyone else's.

- Research records will be kept on a secure computer drive.

- Research records will be destroyed within 5 years.

- Access to the research records will be limited to the researchers.

- However, sometimes, sponsors, funders, regulators, and the University staff who review research may have to look the research records.

What if I choose to not take part or leave the study?

- You are free to not participate or withdraw from the study at any time

- If you choose not to participate, it will not affect your relationship with PSU, your provider, or your support group.

Who can I contact if I have any questions?

- You can call Casadi "Khaki” Marino or Dr. Mary Oschwald who is the researcher in charge of this study. Their number is 503-490-5856.

- If you believe you may have suffered injury or harm from this research, call Dr. Mary Oschwald at (503) 725-9602. She will tell you what to do next.

- If you have any questions about your rights as a person taking part in the study, you may contact: Human Subjects Research Review Committee Office of Research Integrity (HSRRC/ORI), 1600 SW $4^{\text {th }}$ Street, Market Center Building, Portland, OR, phone (503)725-2227.

\section{Statement of Consent:}

- I understand the information in this form.

- My questions have been answered.

- I agree to take part in this study by starting the computer-based survey.

- Starting the survey indicates my voluntary consent to participate

- No one is making me take this survey.

- I understand that I can print off this consent form to keep for my records.

Yes

No 
Once you have completed the survey, you will be able to enter a drawing for one of two $\$ 100$ Visa gift cards. The drawing is optional. To enter, you can follow the link provided at the very end. No identifying information will be transferred between the two surveys. Your responses to this survey and the drawing will be kept separate.

First you will take an early draft of the measure being developed. Then you will take three measures that are already being used: The Recovery Assessment Scale, The Process of Recovery Questionnaire, and the Beck Hopelessness Scale.

\section{Social Recovery Measure}

Casadi "Khaki" Marino

Instructions: This measure is being developed to understand more about the process of recovery. Below is a list of statements that describe how people sometimes think and feel about themselves and their lives. Please read each one and choose the number that best describes how much you agree or disagree with the statement. If it is helpful, think about how you have been doing over the past week or so.

Click to write the question text

\begin{tabular}{l|ccccc}
\hline Disagree & Disagree & $\begin{array}{c}\text { Neither agree } \\
\text { or disagree }\end{array}$ & Agree & Agree Strongly \\
\hline $\begin{array}{l}\text { I feel I belong to a } \\
\text { community. }\end{array}$ & 0 & 0 & 0 & 0 & 0 \\
I am an acceptable person. & 0 & 0 & 0 & 0 & 0 \\
I have let go of the past. & 0 & 0 & 0 & 0 & 0 \\
I have a sense of safety in \\
$\begin{array}{l}\text { community. } \\
\text { I can live in the present }\end{array}$
\end{tabular}


4/18/2016

me for who I really am.

I know what I truly believe.

I know what is true and right for me.

I can express my inner experiences.

I can be my true self.

I have genuine

relationships.

I have people I can trust.

I can be vulnerable.

I am a worthy person.

I can pursue my goals.

I can connect with others.

I have something to offer

others.

I have inner motivation.

I have relationships that are mutually supportive.

I am responsible for myself.

I can accept experiences even if they are difficult.

I choose which

relationships are right for me.

I know what I truly value.

I know people who can understand my

experiences with mental health challenges.

I have a community that recognizes my abilities.

I can cope with mental distress.

I have meaningful things to do.

I have access to the material resources I need.

I know what it is I really

want.

I have hope for the future.

I have a community to which I can contribute.

I have a community that values me.

I have opportunities to talk about how mental health

challenges have affected
Qualtrics Survey Software

$\checkmark$

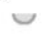

$\checkmark$

O

$\mathrm{O}$

O

0

O

O

O

O

0

0

O

O

O

O

○

O

O

O

○

O

O

O

O

O

O

O

O

$4 / 14$ 
4/18/2016

my life.

I am acceptable to the people who matter to me

I can voice my opinions

know others who can understand how my menta health challenges connect to my life experiences.

I am more than my experiences with mental health challenges.

I can exercise my rights.

I have relationships that inspire hope.

I grew through my experiences with mental health challenges.

I can make sense of my experiences with mental health challenges.

My life is meaningful.

I have relationships in which I am seen as important.

I can be effective.

I can express myself

I have others I can depend on.

I am a valuable person

I can lead a full life.

I can make my own decisions.

have opportunities to grow.

I am a capable person.

I can be optimistic

I am needed by others

I can both give and receive help.

I can make a contribution.

I have relationships in which I am an equal.

I can direct my life.

I can set my own goals.

I have opportunities to make a contribution.

I am responsible for making ttps://portlandstate.qualtrics.com/ControlPanel/Ajax. php?action=GetSurveyPrintPreview

-

O

○

O

O

O

O

O

$\bigcirc$
Qualtrics Survey Software

○

○

○

O

O

O

O

O

O

○

O

O

0

○

○

O

○

○

O

O

○

O

O

O

O

O

○

○

O

0

O

-

$5 / 14$ 


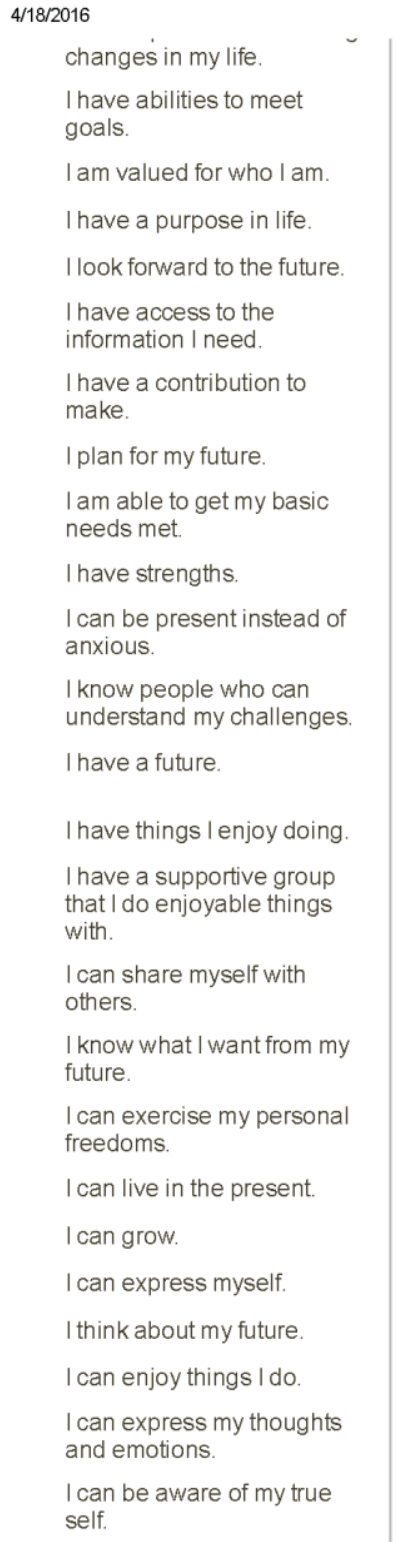

\begin{tabular}{|c|c|c|c|}
\hline & Qualtrics & & \\
\hline u & $u$ & $u$ & $u$ \\
\hline O & 0 & 0 & 0 \\
\hline O & 0 & 0 & 0 \\
\hline 0 & 0 & 0 & 0 \\
\hline 0 & 0 & 0 & 0 \\
\hline 0 & 0 & 0 & 0 \\
\hline 0 & 0 & 0 & 0 \\
\hline O & 0 & 0 & 0 \\
\hline 0 & 0 & 0 & 0 \\
\hline 0 & 0 & 0 & 0 \\
\hline 0 & 0 & 0 & 0 \\
\hline 0 & 0 & 0 & 0 \\
\hline 0 & 0 & 0 & 0 \\
\hline 0 & 0 & 0 & 0 \\
\hline 0 & 0 & 0 & 0 \\
\hline 0 & 0 & 0 & 0 \\
\hline 0 & 0 & 0 & 0 \\
\hline 0 & 0 & 0 & 0 \\
\hline 0 & 0 & 0 & 0 \\
\hline 0 & 0 & 0 & 0 \\
\hline O & 0 & 0 & 0 \\
\hline O & 0 & 0 & 0 \\
\hline 0 & 0 & 0 & 0 \\
\hline 0 & 0 & 0 & 0 \\
\hline 0 & 0 & 0 & 0 \\
\hline
\end{tabular}

Next you will take three measures that have already been developed. First up is the Recovery Assessment Scale.

The Recovery Assessment Scale (Giffort, Schmook, Vollendorf, \& Gervain, 1999) 
Instructions: Below is a list of statements that describe how people sometimes feel about themselves and their lives. Please read each one carefully and chose the number to the right that best describes the extent to which you agree or disagree with the statement. Choose only one number for each statement and do not skip any items.

Click to write the question text

\begin{tabular}{|c|c|c|c|c|c|}
\hline & $\begin{array}{l}\text { Strongly } \\
\text { disagree }\end{array}$ & Disagree & $\begin{array}{l}\text { Neither Agree } \\
\text { nor Disagree }\end{array}$ & Agree & Strongly Agree \\
\hline I have a desire to succeed. & O & 0 & O & 0 & 0 \\
\hline $\begin{array}{l}\text { I have my own plan for how } \\
\text { to stay or become well. }\end{array}$ & O & O & 0 & 0 & 0 \\
\hline $\begin{array}{l}\text { I have goals in life that I } \\
\text { want to reach. }\end{array}$ & O & 0 & 0 & 0 & O \\
\hline $\begin{array}{l}\text { I believe I can meet my } \\
\text { current personal goals. }\end{array}$ & 0 & O & 0 & 0 & O \\
\hline I have a purpose in life. & O & O & O & ○ & 0 \\
\hline $\begin{array}{l}\text { Even when I don't care } \\
\text { about myself, other people } \\
\text { do. }\end{array}$ & O & O & O & O & O \\
\hline $\begin{array}{l}\text { I understand how to control } \\
\text { the symptoms of my mental } \\
\text { illness. }\end{array}$ & O & O & O & O & 0 \\
\hline $\begin{array}{l}\text { I can handle it if I get sick } \\
\text { again. }\end{array}$ & O & 0 & O & 0 & O \\
\hline $\begin{array}{l}\text { I can identify what triggers } \\
\text { the symptoms of my mental } \\
\text { illness. }\end{array}$ & O & O & O & 0 & O \\
\hline $\begin{array}{l}\text { I can help myself become } \\
\text { better. }\end{array}$ & 0 & O & 0 & 0 & 0 \\
\hline $\begin{array}{l}\text { Fear doesn't stop me from } \\
\text { living the way I want to. }\end{array}$ & $\mathrm{O}$ & O & O & O & O \\
\hline $\begin{array}{l}\text { I know that there are mental } \\
\text { health services that do help } \\
\text { me. }\end{array}$ & $\mathrm{O}$ & $\mathrm{O}$ & O & O & O \\
\hline $\begin{array}{l}\text { There are things that I can } \\
\text { do that help me deal with } \\
\text { unwanted symptoms. }\end{array}$ & $\mathrm{O}$ & 0 & 0 & 0 & 0 \\
\hline $\begin{array}{l}\text { I can handle what happens } \\
\text { in my life. }\end{array}$ & 0 & 0 & 0 & 0 & O \\
\hline I like myself. & O & O & O & O & 0 \\
\hline $\begin{array}{l}\text { If people really knew me, } \\
\text { they would like me. }\end{array}$ & $\mathrm{O}$ & 0 & 0 & O & 0 \\
\hline $\begin{array}{l}\text { I am a better person than } \\
\text { before my experience with } \\
\text { mental illness. }\end{array}$ & $\mathrm{O}$ & $\mathrm{O}$ & 0 & O & O \\
\hline $\begin{array}{l}\text { Although my symptoms } \\
\text { may get worse, I know I can } \\
\text { handle it. }\end{array}$ & 0 & 0 & 0 & 0 & 0 \\
\hline If I keep trying, I will & $a$ & $\therefore$ & $a$ & a & $\frown$ \\
\hline
\end{tabular}




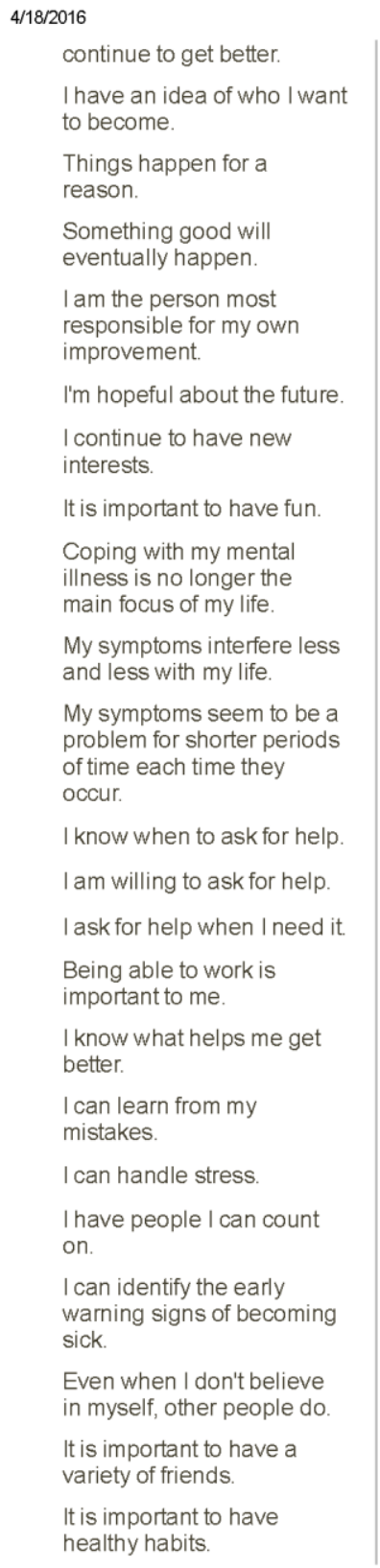

\begin{tabular}{|c|c|c|c|c|}
\hline & Qualtrics & & & \\
\hline$\checkmark$ & $\checkmark$ & $\checkmark$ & $\checkmark$ & U \\
\hline 0 & 0 & 0 & O & O \\
\hline O & 0 & 0 & O & O \\
\hline O & 0 & 0 & 0 & O \\
\hline O & 0 & 0 & 0 & 0 \\
\hline O & 0 & 0 & O & \\
\hline O & 0 & 0 & 0 & O \\
\hline 0 & 0 & 0 & 0 & \\
\hline O & O & 0 & 0 & \\
\hline O & 0 & 0 & O & 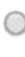 \\
\hline 0 & 0 & 0 & 0 & 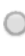 \\
\hline 0 & 0 & 0 & 0 & C \\
\hline O & O & 0 & 0 & $C$ \\
\hline O & 0 & 0 & 0 & C \\
\hline O & 0 & 0 & 0 & C \\
\hline 0 & 0 & 0 & 0 & C \\
\hline 0 & 0 & 0 & 0 & o \\
\hline O & 0 & 0 & 0 & O \\
\hline O & 0 & 0 & 0 & \\
\hline 0 & 0 & 0 & 0 & \\
\hline O & 0 & 0 & 0 & \\
\hline O & 0 & 0 & 0 & \\
\hline 0 & 0 & 0 & 0 & \\
\hline
\end{tabular}

The Process of Recovery Questionnaire (Kilbride, Pitt, Nothard, Welford, Sellwood, \& Morrison, 2009) 
We developed this questionnaire in order to understand more about the process of recovery; what's helpful and what's not helpful. Everyone is different and there will be differences for everyone. The items on this questionnaire were developed through a process of interviewing service users about their recovery journeys. We hope that by filling in this questionnaire you will help us find out information that is important to you and your own recovery. Not all factors will be important to you, since everyone is different. This questionnaire is not intended to be used to impose anything against your wishes.

If you would like to fill in the questionnaire, please take a moment to consider and sum up how things stand for you at the present time, in particular over the last 7 days, with regards to your mental health and recovery.

Please respond to the following statements by choosing the response option that best describes your experience.

Click to write the question text

\begin{tabular}{|c|c|c|c|c|c|}
\hline & $\begin{array}{l}\text { Disagree } \\
\text { strongly }\end{array}$ & Disagree & $\begin{array}{c}\text { Neither agree } \\
\text { nor disagree }\end{array}$ & Agree & Agree Strongly \\
\hline I feel better about myself. & 0 & $\mathrm{O}$ & 0 & 0 & 0 \\
\hline $\begin{array}{l}\text { I feel able to take chances } \\
\text { in life. }\end{array}$ & $\mathrm{O}$ & $\mathrm{O}$ & $\mathrm{O}$ & $\mathrm{O}$ & $\mathrm{O}$ \\
\hline $\begin{array}{l}\text { I am able to develop } \\
\text { positive relationships with } \\
\text { other people. }\end{array}$ & $\bigcirc$ & $\mathrm{O}$ & $\mathrm{O}$ & $\mathrm{O}$ & $\mathrm{O}$ \\
\hline $\begin{array}{l}\text { I feel part of society rather } \\
\text { than isolated. }\end{array}$ & 0 & $\mathrm{O}$ & $\mathrm{O}$ & $\mathrm{O}$ & $\mathrm{O}$ \\
\hline I am able to assert myself. & $\mathrm{O}$ & $\mathrm{O}$ & $\mathrm{O}$ & $\mathrm{O}$ & $\mathrm{O}$ \\
\hline $\begin{array}{l}\text { I feel that my life has a } \\
\text { purpose. }\end{array}$ & 0 & $\mathrm{O}$ & $\mathrm{O}$ & $\mathrm{O}$ & $\mathrm{O}$ \\
\hline $\begin{array}{l}\text { My experiences have } \\
\text { changed me for the better. }\end{array}$ & $\mathrm{O}$ & $\mathrm{O}$ & $\mathrm{O}$ & $\mathrm{O}$ & $\mathrm{O}$ \\
\hline $\begin{array}{l}\text { I have been able to come to } \\
\text { terms with things that have } \\
\text { happened to me in the past } \\
\text { and move on with my life. }\end{array}$ & $\mathrm{O}$ & $\mathrm{O}$ & $\mathrm{O}$ & $\mathrm{O}$ & $\mathrm{O}$ \\
\hline $\begin{array}{l}\text { I am basically strongly } \\
\text { motivated to get better. }\end{array}$ & 0 & 0 & $\mathrm{O}$ & $\mathrm{O}$ & $\mathrm{O}$ \\
\hline $\begin{array}{l}\text { I can recognize the positive } \\
\text { things I have done. }\end{array}$ & 0 & $\mathrm{O}$ & 0 & $\mathrm{O}$ & $\mathrm{O}$ \\
\hline $\begin{array}{l}\text { I am able to understand } \\
\text { myself better. }\end{array}$ & 0 & $\mathrm{O}$ & $\mathrm{O}$ & $\mathrm{O}$ & $\mathrm{O}$ \\
\hline I can take charge of my life. & 0 & 0 & 0 & $\mathrm{O}$ & 0 \\
\hline $\begin{array}{l}\text { I am able to access } \\
\text { independent support. }\end{array}$ & 0 & 0 & 0 & 0 & 0 \\
\hline $\begin{array}{l}\text { I can weigh up the pros and } \\
\text { cons of psychiatric } \\
\text { treatment. }\end{array}$ & 0 & 0 & 0 & 0 & 0 \\
\hline $\begin{array}{l}\text { I feel my experiences have } \\
\text { made me more sensitive } \\
\text { towards others. }\end{array}$ & 0 & 0 & 0 & 0 & 0 \\
\hline $\begin{array}{l}\text { Meeting people who have } \\
\text { had similar experiences } \\
\text { makes me feel better. }\end{array}$ & 0 & 0 & 0 & 0 & 0 \\
\hline
\end{tabular}


4/18/2016

iviy iecuveiy Ilas I Ieipeu challenge other peoples views about getting better.

I am able to make sense of my distressing

experiences.

I can actively engage with life.

I realize that the views of some mental health professionals is not the only way of looking at things.

I can take control of aspects of my life.

I can find the time to do the things I enjoy.
Qualtrics Survey Software

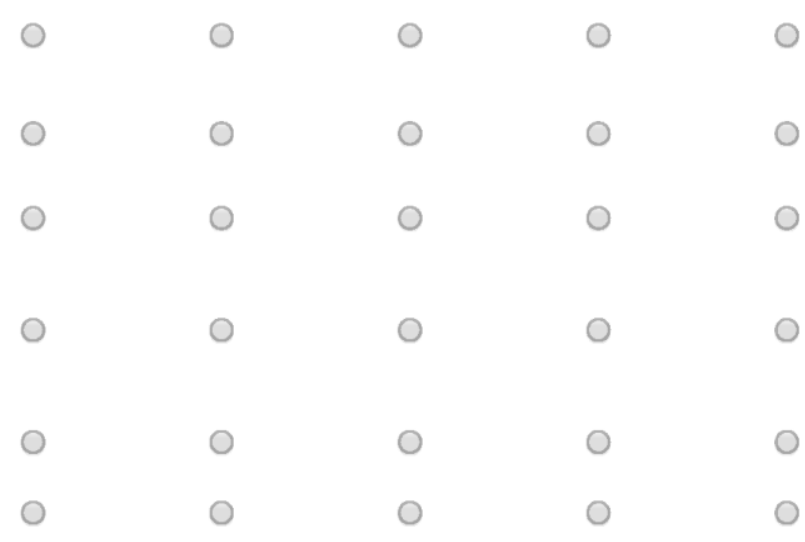

Last measure!

Beck's Hopelessness Scale (Beck, 1974)

This questionnaire consists of a list of twenty statments. Please read the statements carefully one by one. If the statement describes your attitude for the past week, including today, choose "True." If the statement is false for you, choose "False." Please be sure to read each sentence.

Click to write the question text

\begin{tabular}{|c|c|c|}
\hline & True & False \\
\hline $\begin{array}{l}\text { I look forward to the future } \\
\text { with hope and enthusiasm. }\end{array}$ & $\mathrm{O}$ & $\mathrm{O}$ \\
\hline $\begin{array}{l}\text { I might as well give up } \\
\text { because I can't make things } \\
\text { better for myself. }\end{array}$ & $\mathrm{O}$ & $\mathrm{O}$ \\
\hline $\begin{array}{l}\text { When things are going } \\
\text { badly, I'm helped by } \\
\text { knowing they can't stay that } \\
\text { way forever. }\end{array}$ & $\mathrm{O}$ & $\mathrm{O}$ \\
\hline $\begin{array}{l}\text { I can't imagine what my life } \\
\text { would be like in } 10 \text { years. }\end{array}$ & $\mathrm{O}$ & $\mathrm{O}$ \\
\hline $\begin{array}{l}\text { I have enough time to } \\
\text { accomplish the things I most } \\
\text { want to do. }\end{array}$ & $\mathrm{O}$ & $\mathrm{O}$ \\
\hline $\begin{array}{l}\text { In the future, I expect to } \\
\text { succeed in what concerns } \\
\text { me most. }\end{array}$ & $\mathrm{O}$ & $\mathrm{O}$ \\
\hline My future seems dark to me. & $\mathrm{O}$ & $\mathrm{O}$ \\
\hline $\begin{array}{l}\text { l expect to get more of the } \\
\text { good things in life than the } \\
\text { average person. }\end{array}$ & $\mathrm{O}$ & $\mathrm{O}$ \\
\hline $\begin{array}{l}\text { I just don't get the breaks, } \\
\text { and there's no reason to }\end{array}$ & 0 & 0 \\
\hline
\end{tabular}


4/18/2016

believe I will in the future.

My past experiences have prepared me well for my future.

All I can see ahead of me is unpleasantness rather than pleasantness.

I don't expect to get what I really want.

When I look ahead to the future, I expect I will be happier than I am now.

Things just won't work out the way I want them to.

I have great faith in the future.

I never get what I want so it's foolish to want anything.

It is very unlikely that I will get any real satisfaction in the future.

The future seems vague and uncertain to me

I can look forward to more good times than bad times.

There's no use in really

trying to get something I

want because I probably

won't get it.
Qualtrics Survey Software

O

$\mathrm{O}$

O

O

O

$\mathrm{O}$

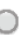

$\mathrm{O}$

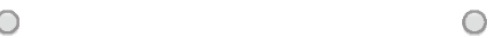

O

O

O

O

O

O

$\mathrm{O}$

0

O

$\mathrm{O}$

Thank you for taking the measures! Now for some questions about your gender, race, etc.

What gender do you identify as?
Male
Female
Transgender/2Spirit
Please provide

What is your age?

$18-24$

25-34

$35-44$

https://portlandstate.qualtrics.com/ControlPanel/Ajax.php?action=GetSurveyPrintPreview 


$\cup_{45-54}^{4 / 18 / 2016}$
$\bigcirc^{55-64}$
$65+$

What is your race/ethnicity?
Caucasian
African American
Asian/Pacific Islander
First Nation//Native American/Alaskan Native
Latino
O Mixed Race
Please provide

What is your partnership status?
Single
In a relationship
Married
Divorced
Widowed
Please provide below

What is your sexual orientation?
Straight/Heterosexual
O LGBTQ
Please provide below
Prefer not to answer

Setting in which you live:
Urban
Small town
Suburb
O Rural/Frontier 
Please provide your zip code

Click to write Choice 1

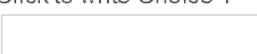

What is your formal educational background?
Less than High Schoo
High School/GED
Trade School
Attended college
AAIAS
BA/BS
Graduate Degree

Your employment status
Working full time
Working part time
Student
Temporarily unemployed
On disability
Retired

Your current and primary mental health diagnosis

Schizophrenia/Schizoaffective/Psychotic Disorder

Bipolar Disorder

Depression

O PTSD

Anxiety Disorder

Dissociative Identity Disorder

Substance Disorder

Please provide below

The type of service or supports that you receive

Traditional community mental health treatment 
Peer services or supports

Both traditional and peer support services

Please provide below

You have reached the end of the survey.

Thank you!

If you would like to enter a drawing for one of two $\$ 100$ Visa gift cards, you can follow the link below. No information from this survey will be transferred. Your responses to this survey will be kept separate from the drawing. (You may need to open the link in a new browser.)

https://portlandstate.qualtrics.com//SE/?SID=SV_9pnvUQ51QxJY27X 


\section{Appendix C}

Skew and Kurtosis of the Items on the Draft SRM

\begin{tabular}{|c|c|c|c|c|}
\hline SRM Item & Skew Statistic & Skew SE & $\begin{array}{l}\text { Kurtosis } \\
\text { Statistic }\end{array}$ & Kurtosis SE \\
\hline $\begin{array}{l}\text { I feel I belong to } \\
\text { a community. }\end{array}$ & -1.198 & .161 & 1.182 & .321 \\
\hline $\begin{array}{l}\text { I am an } \\
\text { acceptable } \\
\text { person. }\end{array}$ & -1.311 & .161 & 2.054 & .321 \\
\hline $\begin{array}{l}\text { I have let go of } \\
\text { the past. }\end{array}$ & -.096 & .161 & -.780 & .321 \\
\hline $\begin{array}{l}\text { I have a sense of } \\
\text { safety in } \\
\text { community. }\end{array}$ & -.715 & .161 & .160 & .321 \\
\hline $\begin{array}{l}\text { I can live in the } \\
\text { present instead of } \\
\text { being focused on } \\
\text { the past. }\end{array}$ & -.706 & .161 & .004 & .321 \\
\hline $\begin{array}{l}\text { I have skills to } \\
\text { interact in } \\
\text { society. }\end{array}$ & -1.261 & .161 & 1.612 & .321 \\
\hline $\begin{array}{l}\text { I am not defined } \\
\text { by my mental } \\
\text { health challenges }\end{array}$ & -.860 & .161 & -.102 & .321 \\
\hline $\begin{array}{l}\text { I value my past } \\
\text { experiences. }\end{array}$ & -1.283 & .161 & 1.736 & .321 \\
\hline $\begin{array}{l}\text { I have a } \\
\text { supportive group } \\
\text { that encourages } \\
\text { me to grow. }\end{array}$ & -1.099 & .161 & 1.211 & .321 \\
\hline $\begin{array}{l}\text { I know people } \\
\text { who accept me } \\
\text { for who I really } \\
\text { am. }\end{array}$ & -1.185 & .161 & 1.536 & .321 \\
\hline $\begin{array}{l}\text { I know what I } \\
\text { truly believe. }\end{array}$ & -1.289 & .161 & 1.694 & .321 \\
\hline $\begin{array}{l}\text { I know what is } \\
\text { true and right for } \\
\text { me. }\end{array}$ & -1.161 & .161 & 1.340 & .321 \\
\hline $\begin{array}{l}\text { I can express my } \\
\text { inner } \\
\text { experiences. }\end{array}$ & -.813 & .161 & .196 & .321 \\
\hline $\begin{array}{l}\text { I can be my true } \\
\text { self. }\end{array}$ & -1.003 & .161 & .847 & .321 \\
\hline $\begin{array}{l}\text { I have genuine } \\
\text { relationships. }\end{array}$ & -.985 & .161 & .665 & .321 \\
\hline
\end{tabular}




\begin{tabular}{|c|c|c|c|c|}
\hline SRM Item & Skew Statistic & \begin{tabular}{|l} 
Skew SE \\
\end{tabular} & $\begin{array}{l}\text { Kurtosis } \\
\text { Statistic }\end{array}$ & Kurtosis SE \\
\hline $\begin{array}{l}\text { I have people I } \\
\text { can trust. }\end{array}$ & -1.364 & .161 & 2.377 & .321 \\
\hline $\begin{array}{l}\text { I can be } \\
\text { vulnerable. }\end{array}$ & -1.037 & .161 & .906 & .321 \\
\hline $\begin{array}{l}\text { I am a worthy } \\
\text { person. }\end{array}$ & -1.240 & .161 & 1.753 & .321 \\
\hline $\begin{array}{l}\text { I can pursue my } \\
\text { goals. }\end{array}$ & -1.006 & .161 & .785 & .321 \\
\hline $\begin{array}{l}\text { I can connect } \\
\text { with others. }\end{array}$ & -1.128 & .161 & 1.314 & .321 \\
\hline $\begin{array}{l}\text { I have something } \\
\text { to offer others. }\end{array}$ & -1.278 & .161 & 2.665 & .321 \\
\hline $\begin{array}{l}\text { I have inner } \\
\text { motivation. }\end{array}$ & -1.065 & .161 & .933 & .321 \\
\hline $\begin{array}{l}\text { I have } \\
\text { relationships that } \\
\text { are mutually } \\
\text { supportive. }\end{array}$ & -1.196 & .161 & 1.574 & .321 \\
\hline $\begin{array}{l}\text { I am responsible } \\
\text { for myself. }\end{array}$ & -.927 & .161 & .649 & .321 \\
\hline $\begin{array}{l}\text { I can accept } \\
\text { experiences even } \\
\text { if they are } \\
\text { difficult. }\end{array}$ & -.721 & .161 & .622 & .321 \\
\hline $\begin{array}{l}\text { I choose which } \\
\text { relationships are } \\
\text { right for me. }\end{array}$ & -.866 & .161 & .994 & .321 \\
\hline $\begin{array}{l}\text { I know what I } \\
\text { truly value. }\end{array}$ & -.898 & .161 & .408 & .321 \\
\hline $\begin{array}{l}\text { I can cope with } \\
\text { mental distress. }\end{array}$ & -.526 & .161 & -.273 & .321 \\
\hline $\begin{array}{l}\text { I have a } \\
\text { community that } \\
\text { recognizes my } \\
\text { abilities. }\end{array}$ & -.803 & .161 & .222 & .321 \\
\hline $\begin{array}{l}\text { I know people } \\
\text { who can } \\
\text { understand my } \\
\text { experiences with } \\
\text { mental health } \\
\text { challenges. }\end{array}$ & -1.095 & .161 & 1.100 & .321 \\
\hline $\begin{array}{l}\text { I have } \\
\text { meaningful } \\
\text { things to do. }\end{array}$ & -1.039 & .161 & .905 & .321 \\
\hline
\end{tabular}




\begin{tabular}{|c|c|c|c|c|}
\hline SRM Item & Skew Statistic & Skew SE & $\begin{array}{l}\text { Kurtosis } \\
\text { Statistic }\end{array}$ & Kurtosis SE \\
\hline $\begin{array}{l}\text { I have access to } \\
\text { the material } \\
\text { resources I need. }\end{array}$ & -.908 & .161 & .583 & .321 \\
\hline $\begin{array}{l}\text { I know what it is } \\
\text { I really want. }\end{array}$ & -.676 & .161 & -.180 & .321 \\
\hline $\begin{array}{l}\text { I have hope for } \\
\text { the future. }\end{array}$ & -1.191 & .161 & 1.125 & .321 \\
\hline $\begin{array}{l}\text { I have a } \\
\text { community to } \\
\text { which I can } \\
\text { contribute. }\end{array}$ & -1.221 & .161 & 1.818 & .321 \\
\hline $\begin{array}{l}\text { I have a } \\
\text { community that } \\
\text { values me. }\end{array}$ & -.850 & .161 & .436 & .321 \\
\hline $\begin{array}{l}\text { I have } \\
\text { opportunities to } \\
\text { talk about how } \\
\text { mental health } \\
\text { challenges have } \\
\text { affected my life. }\end{array}$ & -1.037 & .161 & .626 & .321 \\
\hline $\begin{array}{l}\text { I am acceptable } \\
\text { to the people who } \\
\text { value me. }\end{array}$ & -1.009 & .161 & .995 & .321 \\
\hline $\begin{array}{l}\text { I can voice my } \\
\text { opinions. }\end{array}$ & -1.076 & .161 & 1.099 & .321 \\
\hline $\begin{array}{l}\text { I know others } \\
\text { who can } \\
\text { understand how } \\
\text { my mental health } \\
\text { challenges } \\
\text { connect to my } \\
\text { life experiences. }\end{array}$ & -1.131 & .161 & 1.320 & .321 \\
\hline $\begin{array}{l}\text { I am more than } \\
\text { my experiences } \\
\text { with mental } \\
\text { health } \\
\text { challenges. }\end{array}$ & -1.203 & .161 & 1.416 & .321 \\
\hline $\begin{array}{l}\text { I can exercise my } \\
\text { rights. }\end{array}$ & -1.090 & .161 & 1.277 & .321 \\
\hline $\begin{array}{l}\text { I have } \\
\text { relationships that } \\
\text { inspire hope. }\end{array}$ & -1.012 & .161 & .982 & .321 \\
\hline
\end{tabular}




\begin{tabular}{|c|c|c|c|c|}
\hline SRM Item & Skew Statistic & Skew SE & $\begin{array}{l}\text { Kurtosis } \\
\text { Statistic }\end{array}$ & Kurtosis SE \\
\hline $\begin{array}{l}\text { I grew through } \\
\text { my experiences } \\
\text { with mental } \\
\text { health } \\
\text { challenges. }\end{array}$ & -1.377 & .161 & 1.914 & .321 \\
\hline $\begin{array}{l}\text { I can make sense } \\
\text { of experiences } \\
\text { with mental } \\
\text { health } \\
\text { challenges. }\end{array}$ & -.706 & .161 & -.132 & .321 \\
\hline $\begin{array}{l}\text { My life is } \\
\text { meaningful. }\end{array}$ & -1.074 & .161 & .785 & .321 \\
\hline $\begin{array}{l}\text { I have } \\
\text { relationships in } \\
\text { which I am seen } \\
\text { as important. }\end{array}$ & -1.145 & .161 & 1.470 & .321 \\
\hline $\begin{array}{l}\text { I can be } \\
\text { effective. }\end{array}$ & -1.375 & .161 & 2.754 & .321 \\
\hline $\begin{array}{l}\text { I can express } \\
\text { myself. }\end{array}$ & -1.161 & .161 & 1.340 & .321 \\
\hline $\begin{array}{l}\text { I have others I } \\
\text { can depend on. }\end{array}$ & -1.081 & .161 & 1.154 & .321 \\
\hline $\begin{array}{l}\text { I am a valuable } \\
\text { person. }\end{array}$ & -1.141 & .161 & 1.064 & .321 \\
\hline $\begin{array}{l}\text { I can lead a full } \\
\text { life. }\end{array}$ & -1.032 & .161 & .525 & .321 \\
\hline $\begin{array}{l}\text { I can make my } \\
\text { own decisions. }\end{array}$ & -1.246 & .161 & 2.021 & .321 \\
\hline $\begin{array}{l}\text { I have } \\
\text { opportunities to } \\
\text { grow. }\end{array}$ & -1.289 & .161 & 2.002 & .321 \\
\hline $\begin{array}{l}\text { I am a capable } \\
\text { person. }\end{array}$ & -1.132 & .161 & 1.943 & .321 \\
\hline $\begin{array}{l}\text { I can be } \\
\text { optimistic. }\end{array}$ & -1.186 & .161 & 1.994 & .321 \\
\hline $\begin{array}{l}\text { I am needed by } \\
\text { others. }\end{array}$ & -.838 & .161 & .471 & .321 \\
\hline $\begin{array}{l}\text { I can both give } \\
\text { and receive help. }\end{array}$ & -1.121 & .161 & 1.988 & .321 \\
\hline $\begin{array}{l}\text { I can make a } \\
\text { contribution. }\end{array}$ & -1.325 & .161 & 2.726 & .321 \\
\hline $\begin{array}{l}\text { I have } \\
\text { relationships in } \\
\text { which I am an } \\
\text { equal. }\end{array}$ & -1.012 & .161 & .982 & .321 \\
\hline
\end{tabular}




\begin{tabular}{|c|c|c|c|c|}
\hline SRM Item & Skew Statistic & Skew SE & $\begin{array}{l}\text { Kurtosis } \\
\text { Statistic }\end{array}$ & Kurtosis SE \\
\hline $\begin{array}{l}\text { I can direct my } \\
\text { life. }\end{array}$ & -.755 & .161 & .215 & .321 \\
\hline $\begin{array}{l}\text { I can set my own } \\
\text { goals. }\end{array}$ & -1.248 & .161 & 2.726 & .321 \\
\hline $\begin{array}{l}\text { I have } \\
\text { opportunities to } \\
\text { make a } \\
\text { contribution. }\end{array}$ & -1.312 & .161 & 2.314 & .321 \\
\hline $\begin{array}{l}\text { I am responsible } \\
\text { for making } \\
\text { changes in my } \\
\text { life. }\end{array}$ & -1.549 & .161 & 3.550 & .321 \\
\hline $\begin{array}{l}\text { I have abilities to } \\
\text { meet goals. }\end{array}$ & -1.026 & .161 & 1.360 & .321 \\
\hline $\begin{array}{l}\text { I am valued for } \\
\text { who I am. }\end{array}$ & -.958 & .161 & .780 & .321 \\
\hline $\begin{array}{l}\text { I have a purpose } \\
\text { in life. }\end{array}$ & -.997 & .161 & .712 & .321 \\
\hline $\begin{array}{l}\text { I look forward to } \\
\text { the future. }\end{array}$ & -.947 & .161 & .264 & .321 \\
\hline $\begin{array}{l}\text { I have access to } \\
\text { the information I } \\
\text { need. }\end{array}$ & $\begin{array}{l}-1.018 \\
\end{array}$ & .161 & .933 & .321 \\
\hline $\begin{array}{l}\text { I have a } \\
\text { contribution to } \\
\text { make. }\end{array}$ & -1.392 & .161 & 2.822 & .321 \\
\hline $\begin{array}{l}\text { I plan for my } \\
\text { future. }\end{array}$ & -.810 & .161 & .564 & .321 \\
\hline $\begin{array}{l}\text { I am able to get } \\
\text { my basic needs } \\
\text { met. }\end{array}$ & -1.197 & .161 & 1.580 & .321 \\
\hline I have strengths. & -1.344 & .161 & 3.064 & .321 \\
\hline $\begin{array}{l}\text { I can be present } \\
\text { instead of } \\
\text { anxious. }\end{array}$ & -.610 & .161 & -.077 & .321 \\
\hline $\begin{array}{l}\text { I know people } \\
\text { who can } \\
\text { understand my } \\
\text { mental health } \\
\text { challenges. }\end{array}$ & -.891 & .161 & .968 & .321 \\
\hline I have a future. & -1.122 & .161 & 1.024 & .321 \\
\hline $\begin{array}{l}\text { I have things I } \\
\text { enjoy doing. }\end{array}$ & -1.435 & .161 & 2.522 & .321 \\
\hline
\end{tabular}




\begin{tabular}{|l|l|l|l|l|}
\hline SRM Item & Skew Statistic & Skew SE & $\begin{array}{l}\text { Kurtosis } \\
\text { Statistic }\end{array}$ & Kurtosis SE \\
\hline $\begin{array}{l}\text { I have a } \\
\text { supportive group } \\
\text { that I do } \\
\text { enjoyable things } \\
\text { with. }\end{array}$ & -.624 & .161 & -.405 & .321 \\
\hline $\begin{array}{l}\text { I can share } \\
\text { myself with } \\
\text { others. }\end{array}$ & -.836 & .161 & .428 & .321 \\
\hline $\begin{array}{l}\text { I know what I } \\
\text { want from my } \\
\text { future. }\end{array}$ & -.754 & .161 & .387 & .321 \\
\hline $\begin{array}{l}\text { I can exercise my } \\
\text { personal } \\
\text { freedoms. }\end{array}$ & -.994 & .161 & 1.035 & .321 \\
\hline $\begin{array}{l}\text { I can live in the } \\
\text { present. }\end{array}$ & -.801 & .161 & .672 & .321 \\
\hline I can grow. & -1.348 & .161 & 2.536 & .321 \\
\hline $\begin{array}{l}\text { I think about my } \\
\text { future. }\end{array}$ & -1.271 & .161 & 2.151 & .321 \\
\hline $\begin{array}{l}\text { I can enjoy things } \\
\text { I do. }\end{array}$ & -1.336 & .161 & 3.005 & .321 \\
\hline $\begin{array}{l}\text { I can express my } \\
\text { thoughts and } \\
\text { emotions. }\end{array}$ & -1.186 & .161 & 1.368 & .321 \\
\hline $\begin{array}{l}\text { I can be aware of } \\
\text { my true self. }\end{array}$ & -1.311 & .161 & & \\
\hline
\end{tabular}




\section{Appendix D}

\section{Total Variance Explained and Pattern Matrix}

\section{Total Variance Explained}

II. Pattern Matrix 
I. Total Variance Explained

\begin{tabular}{|c|c|c|c|c|c|c|c|}
\hline \multirow[b]{2}{*}{$\begin{array}{l}\text { Compon } \\
\text { ent }\end{array}$} & \multicolumn{3}{|c|}{ Initial Eigenvalues } & \multicolumn{3}{|c|}{$\begin{array}{c}\text { Extraction Sums of Squared } \\
\text { Loadings }\end{array}$} & \multirow{2}{*}{$\begin{array}{c}\text { Rotation } \\
\text { Sums of } \\
\text { Squared } \\
\text { Loadings } \\
\\
\text { Total } \\
\end{array}$} \\
\hline & $\begin{array}{c}\text { Tota } \\
1\end{array}$ & $\begin{array}{c}\% \text { of } \\
\text { Variance }\end{array}$ & $\begin{array}{c}\text { Cumulative } \\
\%\end{array}$ & Total & $\begin{array}{c}\% \text { of } \\
\text { Varianc } \\
\mathrm{e}\end{array}$ & $\begin{array}{l}\text { Cumulati } \\
\text { ve } \%\end{array}$ & \\
\hline 1 & $\begin{array}{r}38.9 \\
50\end{array}$ & 44.770 & 44.770 & 38.950 & 44.770 & 44.770 & 37.172 \\
\hline 2 & $\begin{array}{r}3.50 \\
7\end{array}$ & 4.031 & 48.801 & 3.507 & 4.031 & 48.801 & 27.508 \\
\hline 3 & $\begin{array}{r}2.74 \\
3\end{array}$ & 3.153 & 51.954 & & & & \\
\hline 4 & $\begin{array}{r}2.56 \\
0\end{array}$ & 2.943 & 54.897 & & & & \\
\hline 5 & $\begin{array}{r}1.96 \\
7\end{array}$ & 2.261 & 57.158 & & & & \\
\hline 6 & $\begin{array}{r}1.86 \\
7\end{array}$ & 2.146 & 59.303 & & & & \\
\hline 7 & $\begin{array}{r}1.73 \\
6\end{array}$ & 1.995 & 61.299 & & & & \\
\hline 8 & $\begin{array}{r}1.63 \\
5\end{array}$ & 1.879 & 63.178 & & & & \\
\hline 9 & $\begin{array}{r}1.49 \\
5\end{array}$ & 1.718 & 64.896 & & & & \\
\hline 10 & $\begin{array}{r}1.36 \\
9\end{array}$ & 1.573 & 66.469 & & & & \\
\hline 11 & $\begin{array}{r}1.34 \\
2\end{array}$ & 1.542 & 68.011 & & & & \\
\hline 12 & $\begin{array}{r}1.16 \\
4\end{array}$ & 1.338 & 69.349 & & & & \\
\hline 13 & $\begin{array}{r}1.13 \\
6\end{array}$ & 1.306 & 70.654 & & & & \\
\hline 14 & $\begin{array}{r}1.08 \\
3\end{array}$ & 1.244 & 71.899 & & & & \\
\hline
\end{tabular}




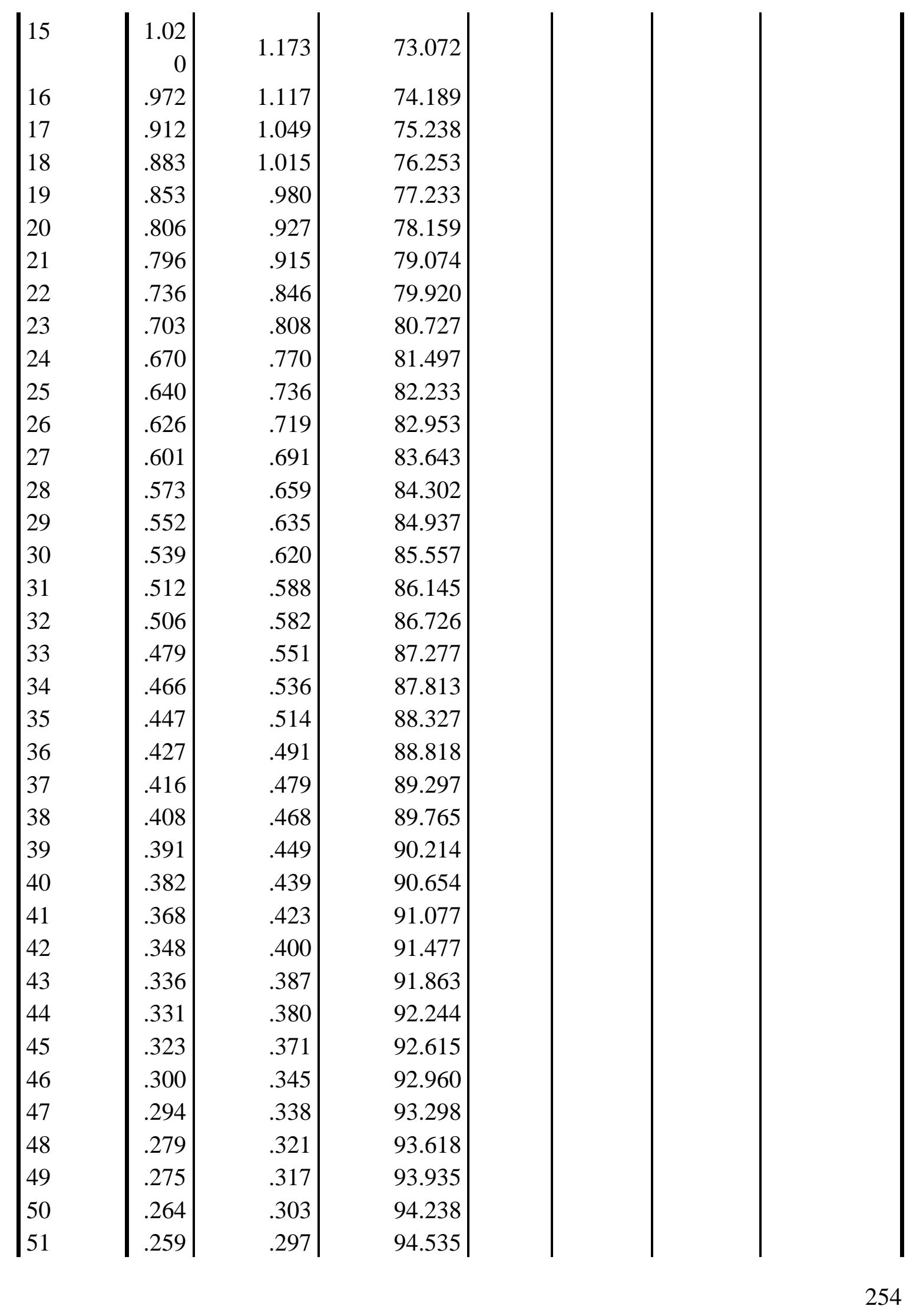




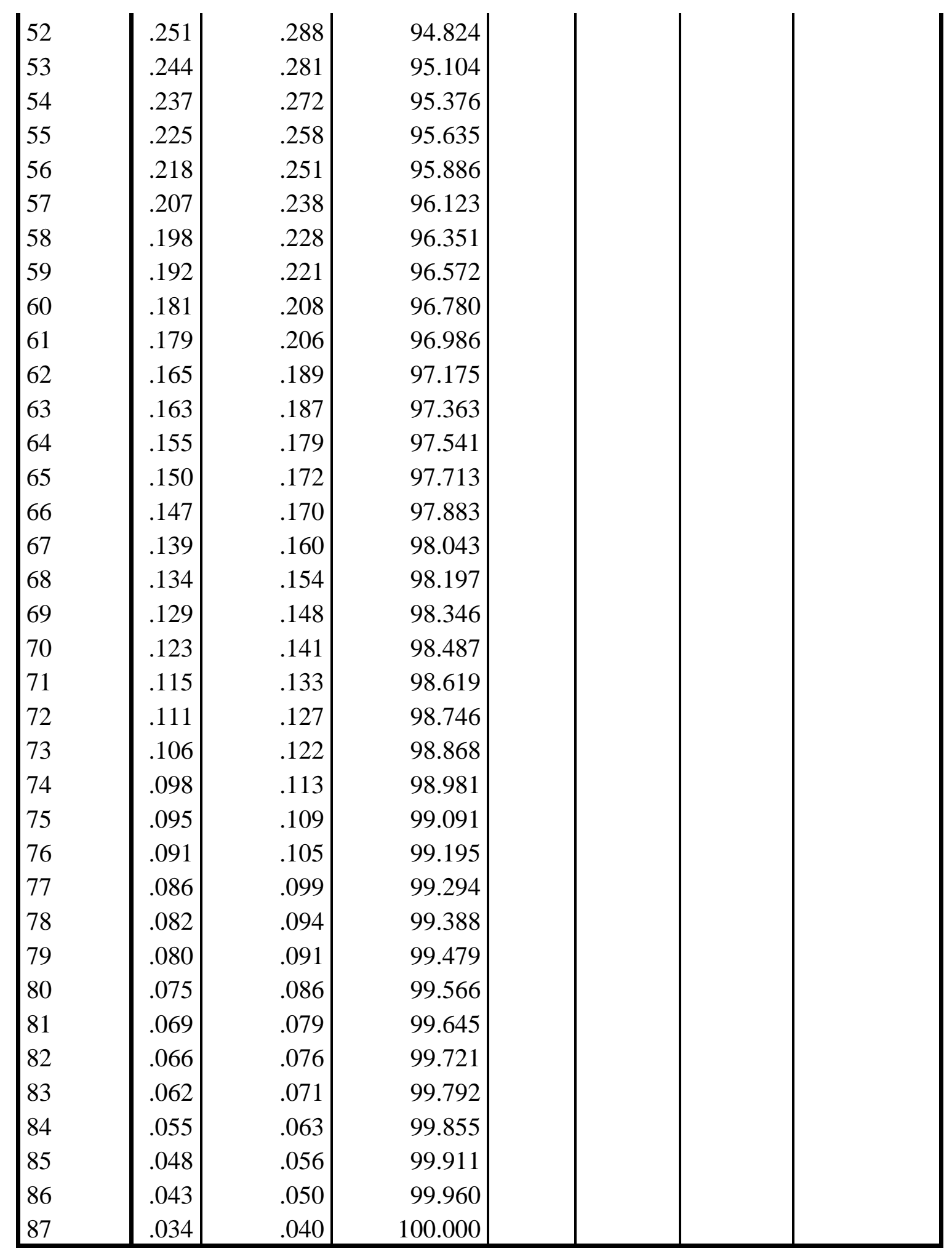

Extraction Method: Principal Component Analysis. 
II. Pattern Matrix

\begin{tabular}{|c|c|c|}
\hline & \multicolumn{2}{|c|}{ Component } \\
\hline & 1 & 2 \\
\hline I can be effective. & .884 & \\
\hline I can be aware of my & 822 & \\
\hline true self. & .832 & \\
\hline I have something to & .815 & \\
\hline offer others. & & \\
\hline $\begin{array}{l}\text { I have a contribution } \\
\text { to make. }\end{array}$ & .813 & \\
\hline $\begin{array}{l}\text { I can set my own } \\
\text { goals. }\end{array}$ & .811 & \\
\hline I am a capable & .808 & \\
\hline person. & & \\
\hline I plan for my future. & .802 & \\
\hline $\begin{array}{l}\text { I can make a } \\
\text { contribution. }\end{array}$ & .799 & \\
\hline I can direct my life. & .796 & \\
\hline $\begin{array}{l}\text { I can exercise my } \\
\text { personal freedoms. }\end{array}$ & .780 & \\
\hline I can be optimistic. & .769 & \\
\hline $\begin{array}{l}\text { I can make my own } \\
\text { decisions. }\end{array}$ & .766 & \\
\hline $\begin{array}{l}\text { I can exercise my } \\
\text { rights. }\end{array}$ & .759 & \\
\hline $\begin{array}{l}\text { I am a valuable } \\
\text { person. }\end{array}$ & .753 & \\
\hline $\begin{array}{l}\text { I look forward to the } \\
\text { future. }\end{array}$ & .745 & \\
\hline I have strengths. & .734 & \\
\hline I can enjoy things I & .727 & \\
\hline $\begin{array}{l}\text { I can be present } \\
\text { instead of anxious. }\end{array}$ & .726 & \\
\hline $\begin{array}{l}\text { I have abilities to } \\
\text { meet goals. }\end{array}$ & .714 & \\
\hline I can lead a full life. & .712 & \\
\hline
\end{tabular}




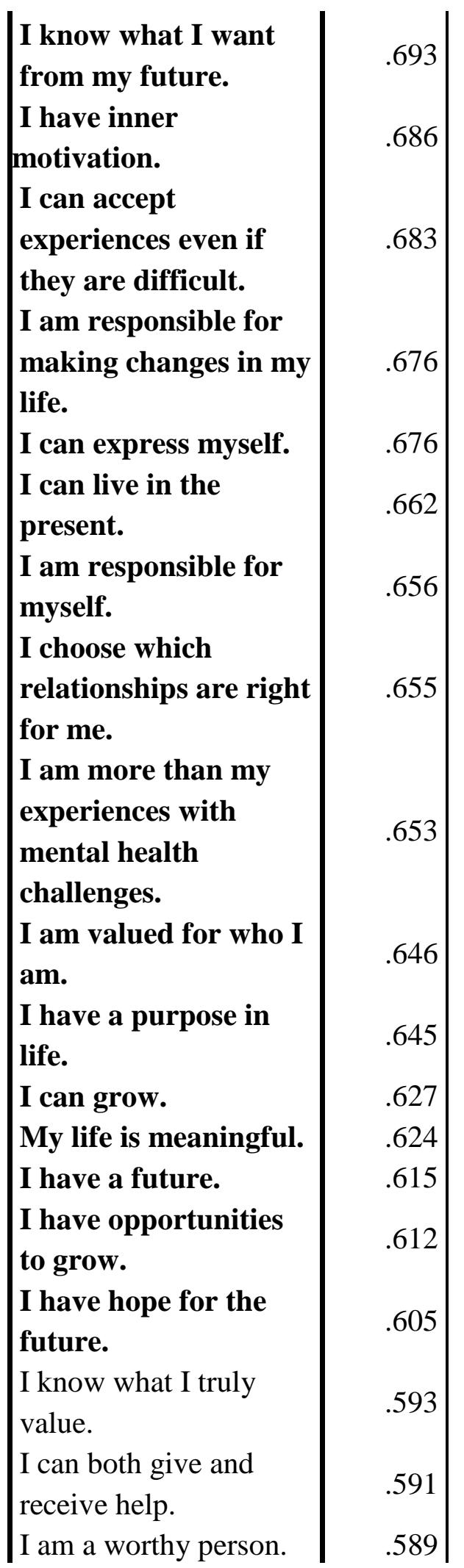




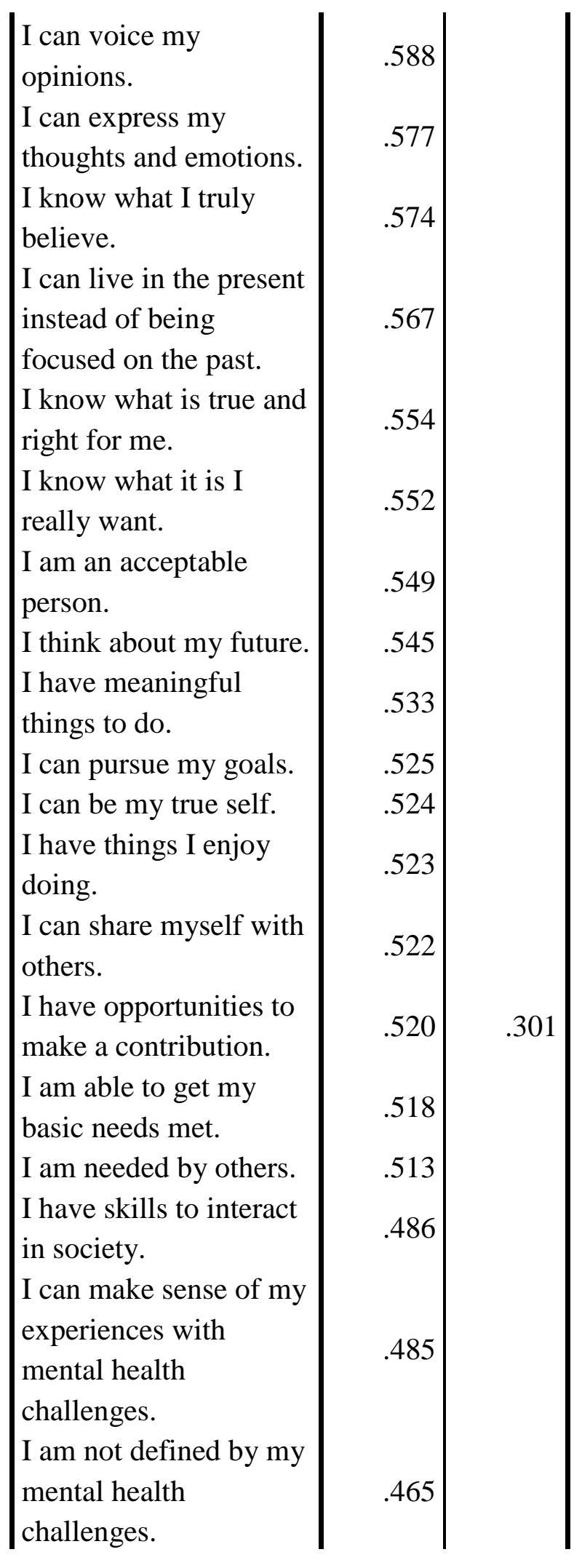




\begin{tabular}{|c|c|c|}
\hline $\begin{array}{l}\text { I have let go of the } \\
\text { past. } \\
\text { I have access to the } \\
\text { information I need. } \\
\text { I have relationships in } \\
\text { which I am an equal. } \\
\text { I can express my inner } \\
\text { experiences. } \\
\text { I can connect with } \\
\text { others. } \\
\text { I can cope with mental } \\
\text { distress. } \\
\text { I have relationships } \\
\text { that are mutually } \\
\text { supportive. } \\
\text { I have a community } \\
\text { that values me. } \\
\text { I feel I belong to a } \\
\text { community. } \\
\text { I have a supportive } \\
\text { group that } \\
\text { encourages me to } \\
\text { grow. } \\
\text { I have a community } \\
\text { that recognizes my } \\
\text { abilities. } \\
\text { I have opportunities } \\
\text { to talk about how } \\
\text { mental health } \\
\text { challenges have } \\
\text { affected my life. } \\
\text { I have people I can } \\
\text { trust. } \\
\text { I can be vulnerable. } \\
\text { I have a supportive } \\
\text { group that I do } \\
\text { enjoyable things with. }\end{array}$ & .438 & $\begin{array}{l}.662 \\
.626 \\
.618\end{array}$ \\
\hline
\end{tabular}




\begin{tabular}{|c|c|c|}
\hline $\begin{array}{l}\text { I have relationships } \\
\text { that inspire hope. } \\
\text { I know others who } \\
\text { can understand how } \\
\text { my mental health } \\
\text { challenges connect to } \\
\text { my life experiences. } \\
\text { I have a sense of safety } \\
\text { in community. } \\
\text { I have genuine } \\
\text { relationships. } \\
\text { I have others I can } \\
\text { depend on. } \\
\text { I have a community to } \\
\text { which I can contribute. } \\
\text { I know people who can } \\
\text { understand my } \\
\text { experiences with } \\
\text { mental health } \\
\text { challenges. } \\
\text { I know people who } \\
\text { accept me for who I } \\
\text { really am. } \\
\text { I have access to the } \\
\text { material resources I } \\
\text { need. } \\
\text { I have relationships in } \\
\text { which I am seen as } \\
\text { important. } \\
\text { I am acceptable to the } \\
\text { people who matter to } \\
\text { me. } \\
\text { I grew through my } \\
\text { experiences with } \\
\text { mental health } \\
\text { challenges. }\end{array}$ & .338 & $\begin{array}{l}.595 \\
.592 \\
.559\end{array}$ \\
\hline
\end{tabular}




\begin{tabular}{|l|r|r|}
$\begin{array}{l}\text { I know people who can } \\
\text { understand my } \\
\text { challenges. }\end{array}$ & .322 & .375 \\
$\begin{array}{l}\text { I value my past } \\
\text { experiences. }\end{array}$ & & .323 \\
\hline
\end{tabular}

Extraction Method: Principal Component Analysis.

Rotation Method: Oblimin with Kaiser Normalization. 


\section{Appendix E}

\section{Social Recovery Measure}

Instructions: Below is a list of statements that describe how people sometimes feel about themselves and their social environments. Please read each one and choose the number that best describes how much you agree or disagree with the statement. Choose only one number for each statement and do not skip any items. If it is helpful, think about how you have been doing over the past week or so.

\begin{tabular}{|c|c|c|c|c|c|}
\hline & $\begin{array}{l}\text { Strongly } \\
\text { disagree }\end{array}$ & Disagree & $\begin{array}{l}\text { Neither } \\
\text { agree or } \\
\text { disagree }\end{array}$ & Agree & $\begin{array}{l}\text { Strongly } \\
\text { agree }\end{array}$ \\
\hline $\begin{array}{l}\text { 1. I have } \\
\text { something } \\
\text { to offer } \\
\text { others. }\end{array}$ & 1 & 2 & 3 & 4 & 5 \\
\hline $\begin{array}{l}\text { 2. I have } \\
\text { relationships } \\
\text { that are } \\
\text { mutually } \\
\text { supportive. }\end{array}$ & 1 & 2 & 3 & 4 & 5 \\
\hline $\begin{array}{l}\text { 3. I am a } \\
\text { capable } \\
\text { person. }\end{array}$ & 1 & 2 & 3 & 4 & 5 \\
\hline $\begin{array}{l}\text { 4. I plan for } \\
\text { my future. }\end{array}$ & 1 & 2 & 3 & 4 & 5 \\
\hline $\begin{array}{l}\text { 5. I have a } \\
\text { community } \\
\text { that values } \\
\text { me. }\end{array}$ & 1 & 2 & 3 & 4 & 5 \\
\hline $\begin{array}{l}\text { 6. I have inner } \\
\text { motivation. }\end{array}$ & 1 & 2 & 3 & 4 & 5 \\
\hline $\begin{array}{l}\text { 7. I can } \\
\text { exercise my } \\
\text { personal } \\
\text { freedoms. }\end{array}$ & 1 & 2 & 3 & 4 & 5 \\
\hline $\begin{array}{l}\text { 8. I feel I } \\
\text { belong to a } \\
\text { community. }\end{array}$ & 1 & 2 & 3 & 4 & 5 \\
\hline
\end{tabular}




\begin{tabular}{|c|c|c|c|c|c|}
\hline & $\begin{array}{c}\text { Strongly } \\
\text { disagree }\end{array}$ & Disagree & $\begin{array}{c}\text { Neither } \\
\text { agree or } \\
\text { disagree }\end{array}$ & Agree & $\begin{array}{c}\text { Strongly } \\
\text { agree }\end{array}$ \\
\hline $\begin{array}{c}\text { 9. I can lead a } \\
\text { full life. }\end{array}$ & 1 & 2 & 3 & 4 & 5 \\
\hline $\begin{array}{c}\text { 10. I have } \\
\text { people I can } \\
\text { trust. }\end{array}$ & 1 & 2 & 3 & 4 & 5 \\
\hline $\begin{array}{c}\text { 11. I am a } \\
\text { valuable } \\
\text { person. }\end{array}$ & 1 & 2 & 3 & 4 & 5 \\
\hline $\begin{array}{c}\text { 12. I have } \\
\text { relationships } \\
\text { that inspire } \\
\text { hope. }\end{array}$ & 1 & 2 & 3 & 4 & 5 \\
\hline $\begin{array}{c}\text { 13. I have } \\
\text { strengths. }\end{array}$ & 1 & 2 & 3 & 4 & 5 \\
\hline $\begin{array}{c}\text { 14. I can enjoy } \\
\text { things I do. }\end{array}$ & 1 & 2 & 3 & 4 & 5 \\
\hline $\begin{array}{c}\text { 15. I have a } \\
\text { community } \\
\text { that } \\
\text { recognizes } \\
\text { my abilities. }\end{array}$ & 1 & 2 & 3 & 4 & 5 \\
\hline $\begin{array}{c}\text { 16. I have a } \\
\text { purpose in } \\
\text { life. }\end{array}$ & 1 & 2 & 3 & 4 & 5 \\
\hline $\begin{array}{c}\text { 17. I have } \\
\text { bilities to } \\
\text { meet goals. }\end{array}$ & 1 & 2 & 3 & 4 & 5 \\
\hline $\begin{array}{c}\text { 18. I have a } \\
\text { supportive } \\
\text { group that } \\
\text { encourages } \\
\text { me to grow. }\end{array}$ & 1 & 2 & 3 & 4 & 5 \\
\hline $\begin{array}{c}\text { 19. I am valued } \\
\text { for who I } \\
\text { am. }\end{array}$ & 1 & & & & \\
\hline
\end{tabular}

